(c) 2005 International Press

Adv. Theor. Math. Phys. 9 (2005) 775-860

\title{
Instantons, fluxons, and open gauge string theory
}

\section{Luca Griguolo ${ }^{1}$, Domenico Seminara ${ }^{2}$, and Richard J. Szabo ${ }^{3}$}

${ }^{1}$ Dipartimento di Fisica, Università di Parma, INFN Gruppo Collegato di Parma, Parco Area delle Scienze 7/A, 43100 Parma, Italy griguolo@fis.unipr.it

${ }^{2}$ Dipartimento di Fisica, Polo Scientifico Università di Firenze, INFN Sezione di Firenze, Via G. Sansone 1, 50019 Sesto Fiorentino, Italy seminara@fi.infn.it

${ }^{3}$ Department of Mathematics, Heriot-Watt University, Scott Russell Building, Riccarton, Edinburgh EH14 4AS, UK

r.j.szabo@ma.hw.ac.uk

\begin{abstract}
We use the exact instanton expansion to illustrate various string characteristics of non-commutative gauge theory in two dimensions. We analyse the spectrum of the model and present some evidence in favour of Hagedorn and fractal behaviours. The decompactification limit of noncommutative torus instantons is shown to map in a very precise way, at both the classical and quantum levels, onto fluxon solutions on the non-commutative plane. The weak-coupling singularities of the usual Gross-Taylor string partition function for QCD on the torus are studied
\end{abstract}

e-print archive: http://lanl.arXiv.org/abs/hep-th/0411277 
in the instanton representation and its double-scaling limit, appropriate for the mapping onto non-commutative gauge theory, is shown to be a generating function for the volumes of the principal moduli spaces of holomorphic differentials. The non-commutative deformation of this moduli space geometry is described and appropriate open string interpretations are proposed in terms of the fluxon expansion.

\section{Contents}

1. Introduction and summary

2. Non-commutative instantons in two dimensions

3. The spectrum of non-commutative instantons

4. Fluxon contributions

4.1. Decompactification onto fluxons

4.2. Decompactification of instanton configurations

4.3. Properties of fluxons

801

4.4. The fluxon partition function

803

5. Instanton string expansions

804

5.1. Gross-Taylor series on the torus

805

5.2. Instanton expansion of physical $U(N)$ gauge theory

809

5.3. Saddle point solution

811

5.4. Quasi-modularity of higher instanton configurations

816

6. The double-scaling limit

819

6.1. Saddle point solution 820

6.2. Asymptotics of simple Hurwitz numbers 825

6.3. Principal moduli spaces of holomorphic differentials 827

6.4. Open string interpretation 
7. Non-commutative instanton string representation 836

7.1. Instanton expansion of $\mathrm{SU}(N) / \mathbb{Z}_{N}$ gauge theory 837

\begin{tabular}{ll} 
7.2. & Double-scaling limit \\
\hline
\end{tabular}

7.3. Weak and strong coupling limits 842

7.4. Open string interpretation 845

$\begin{array}{ll}\text { Acknowledgments } & 846\end{array}$

$\begin{array}{ll}\text { A. Properties of the Jonquière function } & 847\end{array}$

$\begin{array}{ll}\text { A.1. Analytic continuation } & 847\end{array}$

$\begin{array}{lll}\text { A.2. Asymptotic expansion } & 847\end{array}$

$\begin{array}{ll}\text { B. Free fermion representation } & 849\end{array}$

$\begin{array}{ll}\text { References } & 854\end{array}$

\section{Introduction and summary}

One of the most challenging problems in modern theoretical high-energy physics is to derive an effective string theory description for the quantum dynamics of strong interactions. Many interesting approaches were triggered by the observation that the Feynman diagram expansion of non-abelian gauge theories, in the limit of a large number of colours $N$, is naturally organized as in the genus expansion of a string theory [1]. Although a four-dimensional QCD has always resisted any precise string interpretation, there exist a number of examples, such as $\mathcal{N}=4$ supersymmetric YangMills theory in four dimensions [2], two-dimensional QCD [3-5], and ChernSimons gauge theory on $\mathbb{S}^{3}[6]$, in which the project has been effectively worked out. In all these models, the string description appears in the large $N$ limit, confirming the expectation that an effective dynamics of extended objects emerges when the rank of the underlying gauge group goes to infinity.

Non-commutative gauge theories on Moyal-deformed spacetimes (see [7-9] for reviews) describe the low-energy dynamics of D-branes in a constant $B$-field background [10] and their local symmetry group is a particular realization of the infinite unitary group $U(\infty)$ that includes spacetime translations and more generally symplectomorphisms of the flat spacetime [11]. 
These theories encode from the very beginning the concept of large $N$ in a well-defined mathematical framework and therefore seem to be a promising arena in which to tackle the difficult problem of deriving a gauge string dynamics. There are various indications that this framework could be easier than in the ordinary (commutative) case. Non-commutative field theories are in general naturally induced in string theory and they possess many unconventional stringy properties themselves reflecting the non-locality that their interactions retain $[8,9]$. A first important observation is that, in the limit of large non-commutativity parameter $\theta$, the perturbative expansion nicely organizes itself into planar and non-planar Feynman diagrams in exactly the same way that the large $N$ expansion of multicolour field theories does. Moreover, they can be represented and analysed exactly as matrix models, indicating a potential connection with non-critical strings. Their fundamental degrees of freedom are electric dipoles, extended rigid rods whose lengths are proportional to their momenta, and their interactions are therefore governed by string-like mechanisms. Finally, some of these theories admit novel soliton and instanton solutions which have no counterparts in their commutative cousins and can be naturally interpreted as D-branes.

These aspects become particularly interesting in two dimensions, because ordinary Yang-Mills theory on a Riemann surface has a very precise interpretation as a string theory $[3-5]$, while the exact solution of gauge theory on the non-commutative torus $\mathbb{T}_{\theta}^{2}$ has been recently presented through an expansion in non-commutative instantons in $[12,13]$. An explicit realization of two-dimensional non-commutative gauge theory as a matrix model has also been constructed in $[14,15]$ by exploiting the general relation [16] between its lattice regularization and the twisted Eguchi-Kawai (TEK) model [17-19]. According to the general paradigm of reduced models, ordinary $\mathrm{QCD}_{2}$ at large $N$ should be recovered from the TEK model as well. The exact solution of gauge theory on the fuzzy sphere, and its connection to instantons, has also been described via a matrix model representation in [20]. Moreover, concrete computations of observables can be performed in a rather explicit way in these settings. For these reasons, a formulation of two-dimensional non-commutative gauge theory as a string theory appears to be a fruitful line of attack and some interesting relations between large $N$ gauge dynamics, instantons, and strings can be expected to emerge.

In this paper, we present the results of an intensive investigation of two-dimensional Yang-Mills theory on the torus at large $N$ and its noncommutative cousin, with particular emphasis on its string and instanton aspects. Our main technical tool for connecting commutative and noncommutative theories is Morita equivalence [9], which among other things 
implies that when the non-commutativity parameter $\theta=n / N$ is a rational number, then Yang-Mills theory on $\mathbb{T}_{\theta}^{2}$ is exactly equivalent to ordinary gauge theory on $\mathbb{T}^{2}=\mathbb{T}_{\theta=0}^{2}$ with gauge group $\mathrm{SU}(N)$ in a specific sector of magnetic flux. Its fruitfulness lies in the fact that a generic irrational noncommutative gauge theory can be obtained as the limit of commutative ones as $n, N \rightarrow \infty$ with $\theta=n / N$ fixed [21-24]. This suggests that great insight into the string representations of non-commutative gauge theories could be obtained from their commutative counterparts. However, the limit required is not the usual 't Hooft limit, as Morita equivalence implies that the area of the torus must also be scaled with $N$, forcing a double-scaling limit. This is the same sort of large $N$ limit required of the TEK model in order to reproduce continuum non-commutative Yang-Mills theory [16]. We have adapted this technique to describe gauge theory on the non-commutative plane, performing accordingly the decompactification limit of the torus theory [25]. The partition function becomes dominated by contributions from fluxons in this case, the classical solutions on the non-commutative plane which have a direct description in terms of D-branes [26-30]. Remarkably, we are able to describe the correct mathematics underlying the emergence of fluxons from non-commutative torus instantons. But the most surprising results are obtained by applying the double-scaling limit, implied by the Morita equivalence, to the complete commutative $U(N)$ gauge theory on $\mathbb{T}^{2}$. We will find that the partition function is dominated, in this limit, by string states of infinite winding number and it provides a generating function for the volumes of the principal moduli spaces of holomorphic differentials on Riemann surfaces [31]. Our approach, based on a simple saddle-point equation, represents a very quick and efficient way to extract exact formulas for these volumes which avoids the cumbersome combinatorial techniques usually employed in the mathematics literature. The appearence of a rather peculiar moduli space suggests the presence of some particular topological string theory behind this limit, and its generalization to the flux case, relevant for the non-commutative gauge theory, seems to lead to even more exotic geometrical structures.

This paper is organized as follows. Section 2 reviews the structure of instantons on the non-commutative torus and by presenting the partition function for gauge theory on a fixed Heisenberg module, written as a sum over the non-commutative instantons. Section 3 discusses a number of stringy features exhibited by the non-commutative instantons on $\mathbb{T}^{2}$. We illustrate the exponential behaviour in energy of the asymptotic distribution of instanton solutions, and the possibility of a Hagedorn-type phase transition in the theory is briefly addressed. We also present some arguments for a fractal description of the instanton spectrum. In Section 4, we turn our attention to gauge theory on the non-commutative plane which can 
be analysed in detail. Section 4.1 describes the decompactification limit of the torus partition function and how it can be written as a semi-classical expansion around fluxons, Section 4.2 rigorously proves using the language of projective modules that torus instantons map in a very precise way onto fluxons, Section 4.3 illustrates how fluxon characteristics are naturally inherited from the torus instantons, and Section 4.4 presents the resummation of the semi-classical expansion of the partition function on the non-commutative plane. The Gross-Taylor string expansion of commutative $U(N)$ YangMills theory on the torus is revisited in Section 5 and its manifestation in the instanton series is analysed. Section 5.1 describes the singular small area behaviour of the genus expansion, Section 5.2 derives the dual instanton expansion, Section 5.3 derives a saddle-point equation neglecting higher instanton contributions that controls the weak-coupling limit of the string expansion, and Section 5.4 explores in detail the quasi-modular property of the full string partition function in terms of the instanton contributions at weak-coupling. Section 6 analyses the $U(N)$ gauge theory in the doublescaling limit. Section 6.1 gives the general solution of the saddle-point equation and the structure of the exact free energy, Section 6.2 relates the terms in the saddle-point expansion to the asymptotic growth of simple Hurwitz numbers, Section 6.3 explores the geometrical structure behind this limit in terms of the principal moduli spaces of holomorphic differentials, and Section 6.4 discusses the open string interpretation of these results. Finally, in Section 7 we come back to the non-commutative setting and analyse the double-scaling limit of $\mathrm{SU}(N) / \mathbb{Z}_{N}$ gauge theory on $\mathbb{T}^{2}$. Section 7.1 derives the generalization of the instanton expansion in this case, Section 7.2 performs the double-scaling limit illustrating how the chiral fluxon expansion emerges, Section 7.3 shows that the fluxon expansion always dominates the theory thus verifying the absence of phase transitions, and Section 7.4 proposes an open string interpretation of these results. Appendix A. contains some additional technical details, while Appendix B. presents a free fermion representation of the double-scaling limit.

\section{Non-commutative instantons in two dimensions}

In this section, we will briefly describe the structure of instantons on the noncommutative torus. They are defined as solutions of the non-commutative Yang-Mills equations on a square torus $\mathbb{T}^{2}$ of area $A$ which are not gauge transformations of the trivial connection. For this, we will first recall the notion of Heisenberg modules, as they classify all possible types of gauge theories on the non-commutative torus. Further details may be found in [12]. 
Let $\mathcal{A}=\mathcal{S}\left(\mathbb{T}_{\theta}^{2}\right)$ be the algebra of Schwartz functions on the noncommutative torus of deformation parameter $\theta \in \mathbb{R}$ and let $\mathbb{M}_{n}(\mathcal{A})$ denote the algebra of $n \times n$ matrices with entries in $\mathcal{A}$. For any $n \in \mathbb{N}$ and any projector $\mathrm{P} \in \mathbb{M}_{n}(\mathcal{A})$ with $\mathrm{P}^{2}=\mathrm{P}=\mathrm{P}^{\dagger}$, the vector space $\mathcal{E}=\mathrm{P} \mathcal{A}^{n}$ is a finitely generated projective module over the algebra $\mathcal{A}$. Yang-Mills theory on the non-commutative torus is constructed on a fixed stable equivalence class of projective modules. Since the K-theory of the non-commutative torus is $\mathrm{K}^{0}\left(\mathbb{T}_{\theta}^{2}\right)=\mathbb{Z}^{2} \cong \mathbb{Z}+\mathbb{Z} \theta$ (for $\theta$ irrational), any projector $\mathrm{P}=\mathrm{P}_{p, q}$ is characterized by a pair of integers $(p, q)$. Stable modules

$$
\mathcal{E}_{p, q}=\mathrm{P}_{p, q} \mathcal{A}^{n}
$$

live in the restriction to the positive cone of $\mathrm{K}^{0}\left(\mathbb{T}_{\theta}^{2}\right)$ defined by positivity of the corresponding Murray-von Neumann dimension

$$
\operatorname{dim} \mathcal{E}_{p, q}:=\operatorname{Tr} \mathrm{P}_{p, q}=p-q \theta>0 .
$$

Such modules $\mathcal{E}_{p, q}$ are called Heisenberg modules, and on them the YangMills action can be defined. The integer $q$ is the Chern number of the corresponding gauge bundle and it can be computed explicitly in terms of the curvature of the module [12]. The rank of $\mathcal{E}_{p, q}$ is the positive integer

$$
\operatorname{rank} \mathcal{E}_{p, q}:=\operatorname{gcd}(p, q) \text {. }
$$

Any finitely generated projective module over the non-commutative torus is of the form $\left(\mathcal{E}_{p, q}\right)^{l} \oplus \mathcal{A}^{m}$ for some $(p, q) \in \mathbb{Z}^{2}$ and $l, m \in \mathbb{N}_{0}$. We will always assume in the following that $q \neq 0$ and that all modules have been implicitly completed into separable Hilbert spaces using the natural inner products on the vector spaces involved.

The characteristic feature of any Heisenberg module $\mathcal{E}_{p, q}$ is that it always admits an anti-Hermitian connection $\nabla$ of constant curvature

$$
F_{p, q}:=\left[\nabla_{1}, \nabla_{2}\right]=\frac{2 \pi \mathrm{i}}{A} \frac{q}{p-q \theta} \mathrm{P}_{p, q} .
$$

The curvature (2.4) is proportional to the identity element of the endomorphism algebra of (2.1), because the projector $\mathrm{P}_{p, q}$ clearly acts as the identity operator on the module, $\mathrm{P}_{p, q}|\psi\rangle=|\psi\rangle \forall|\psi\rangle \in \mathcal{E}_{p, q}$. From this fact, one may exhibit $\mathcal{E}_{p, q}$ explicitly as the separable Hilbert space

$$
\mathcal{E}_{p, q}=\mathcal{F} \otimes \mathcal{W}_{p, q}
$$

The space $\mathcal{F} \cong L^{2}(\mathbb{R})$ is the Schrödinger representation of the Heisenberg commutation relation (2.4) and it is the unique irreducible module over the Heisenberg Lie algebra. The finite-dimensional vector space $\mathcal{W}_{p, q} \cong \mathbb{C}^{|q|}$ is 
the $|q| \times|q|$ unitary representation of the Weyl-'t Hooft algebra

$$
\Gamma_{1} \Gamma_{2}=\mathrm{e}^{2 \pi \mathrm{i} p / q} \Gamma_{2} \Gamma_{1},
$$

whose unique irreducible module has dimension $|q| / \operatorname{rank} \mathcal{E}_{p, q}$. The unitary generators $U_{i}, i=1,2$ of the non-commutative torus, defined by the commutation relation

$$
U_{1} U_{2}=\mathrm{e}^{2 \pi \mathrm{i} \theta} U_{2} U_{1},
$$

can then be represented in $(2.5)$ as

$$
U_{i}=\mathrm{e}^{\sqrt{A}(p-q \theta) \nabla_{i} / q} \bigotimes \Gamma_{i}, \quad i=1,2 .
$$

Within this framework, it is also possible to classify all critical points of the non-commutative Yang-Mills action on a fixed Heisenberg module $\mathcal{E}_{p, q}[12]$. The constant curvature connection $\nabla$, obeying (2.4), solves the equations of motion and yields the absolute minimum value of the action on $\mathcal{E}_{p, q}$. It can also be used to construct all solutions of the classical equations of motion. The main observation is that, in a neighbourhood of a solution of the Yang-Mills equations, the module $\mathcal{E}_{p, q}$ may be regarded as a direct sum of submodules, and a critical point is always gauge equivalent to the direct sum of the corresponding absolute minima provided by the constant curvature connections on the submodules [12]. The classification of classical solutions is equivalent to finding all possible decompositions of the original Heisenberg module $\mathcal{E}_{p, q}$ into submodules $\mathcal{E}_{p_{k}, q_{k}}$ of the form

$$
\mathcal{E}_{p, q}=\bigoplus_{k} \mathcal{E}_{p_{k}, q_{k}} .
$$

For any given value of $\theta$, a classical solution of Yang-Mills theory on $\mathcal{E}_{p, q}$ is therefore completely characterized by the sets of pairs of integers $\underline{(p, q)}:=$ $\left\{\left(p_{k}, q_{k}\right)\right\}$ satisfying the three constraints

$$
\begin{aligned}
p_{k}-q_{k} \theta & >0, \\
\sum_{k}\left(p_{k}-q_{k} \theta\right) & =p-q \theta, \\
\sum_{k} q_{k} & =q .
\end{aligned}
$$

The collection $\underline{(p, q)}$ is called a partition of the topological numbers $(p, q)$ of the Heisenberg module $\mathcal{E}_{p, q}$. The third equation is the requirement that the Chern number of the module be equal to the total magnetic flux of the direct sum decomposition. When the non-commutativity parameter $\theta$ is irrational, this constraint is an automatic consequence of the second one, while in the rational case it is understood as the requirement of K-theory charge conservation. 
The Yang-Mills action, evaluated on a solution of the classical equations of motion, is computed in terms of the non-commutativity parameter $\theta$ and of a partition $(p, q)$ as

$$
S(\underline{(p, q)} ; \theta)=\frac{2 \pi^{2}}{g^{2} A} \sum_{k} \frac{q_{k}^{2}}{p_{k}-q_{k} \theta},
$$

where $g$ is the Yang-Mills coupling constant and the sum runs through all components $\left(p_{k}, q_{k}\right)$ of $(p, q)$. At the quantum level, the significance of these non-commutative instantons is that the partition function and observables of non-commutative gauge theory in two dimensions are given exactly by their semi-classical approximations and can be represented as sums over classical solutions $[12,13]$. This leads to explicit weak-coupling expansions over partitions. In particular, the partition function on a fixed Heisenberg module $\mathcal{E}_{p, q}$ can be written as

$$
Z_{p, q}\left(g^{2} A, \theta\right)=\sum_{\substack{\text { partitions } \\(\underline{p, q)}}} \frac{(-1) \underline{|(p, q)|}}{\prod_{a} \nu_{a} !} \prod_{k=1}^{|(p, q)|} \sqrt{\frac{2 \pi^{2}}{g^{2} A\left(p_{k}-q_{k} \theta\right)^{3}}} \mathrm{e}^{-S(\underline{(p, q) ; \theta)}}
$$

where the integer $\nu_{a}>0$ is the number of partition components $\left(p_{k}, q_{k}\right) \in$ $\underline{(p, q)}$ which have the ath least dimension $p_{a}-q_{a} \theta$, while $|(p, q)|:=\nu_{1}+$ $\overline{\nu_{2}+} \cdots$ is the total number of components in the partition. This definition requires us to order components of partitions according to their increasing Murray-von Neumann dimensions $p_{k}-q_{k} \theta$ and regard any two partitions as equivalent if they differ only in their partial orderings. In other words, the sum in (2.12) is implicitly assumed to run over unordered partitions. By resumming (2.12) over gauge inequivalent partitions, we may express it as a sum over unstable instantons [12]. The exponential prefactors then represent the exact corrections due to quantum fluctuations about each instanton.

\section{The spectrum of non-commutative instantons}

We will now proceed to demonstrate that the non-commutative instantons on the torus exhibit a number of stringy features which will direct the way towards the string interpretation of the gauge theory partition function (2.12). The simplest way to understand this stringiness is through the strong coupling expansion of the non-commutative gauge theory, which may be expressed as a sum over non-local electric dipole configurations [13]. In this sense, the instanton expansion is dual to an open string representation 
of the gauge theory, in which the endpoints of strings are coupled to a background magnetic field $B=(A \theta / 2 \pi)^{-1}$ and the strings become polarized as dipoles. However, in contrast to the instanton series, the dipole expansion is not very explicit and is difficult to deal with analytically. In what follows, we will attempt to extract the string behaviour directly from the instanton series, and among other things this will yield a direct connection with the conventional string picture of ordinary $\mathrm{QCD}_{2}$, in which the exact partition function can be interpreted as a sum over two-dimensional ramified covers of the underlying torus $\mathbb{T}^{2}$. In this section, we will analyse the spectrum of the non-commutative gauge theory and extract its high-energy asymptotic behaviour.

We start by discussing the structure of the asymptotic density of states in two-dimensional non-commutative gauge theory. The key to extracting string characteristics is to note the extreme differences in the nature of the partitions which contribute to the instanton sum in the cases of rational and irrational values of the non-commutativity parameter $\theta$ [12]. In the rational case, the contributing partitions have module dimensions which are a priori bounded from below, with the fixed bound depending on the value of the rational number $\theta$, while in the irrational case partitions of arbitrarily small dimension always contribute. We can see this difference qualitatively by plotting the possible values of the instanton action (2.11) on a fixed Heisenberg module $\mathcal{E}_{p, q}$. In a $2+1$-dimensional setting, we may also interpret these values as Yang-Mills energies and we will frequently adopt this point of view in the following.

For example, figure 1 illustrates the typical pattern of energies in the case of rational $\theta$ from the contributions of two-component partitions. The vertical axis is the energy, while the horizontal axis is the list of allowed partitions $(p, q)=\left\{\left(p_{1}, q_{1}\right),\left(p_{2}, q_{2}\right)\right\}$ which contribute. The plot traces out the positions of the energies on this list. It is also possible to obtain similar plots for higher component partitions by choosing an appropriate (lexicographic) ordering to arrange partitions along the horizontal axis. As one can see, a very regular pattern emerges, as there are only a small number of well-defined trajectories in this case. Of course, by Morita equivalence, this is the typical situation in ordinary Yang-Mills theory. Since the gauge theory in this case is topological and thereby possesses only finitely many degrees of freedom, we may interpret the energy spectrum as that of some quantum mechanical system consisting of different species of non-interacting particles in a box. The full spectrum can be decomposed as the sum of spectra from different classes of quantum mechanical systems, the collection of which is in a one-to-one correspondence with the partitions of $p-q \theta$ in this case. 




Figure 1: Allowed energies for two-component partitions of the Heisenberg module $\mathcal{E}_{2,2}$ for $\theta=1 / 5$.

In striking contrast, the corresponding situation for irrational $\theta$ is depicted in figure 2. Now the energy points tend to fill the whole plane. This is especially evident in the plots as one starts increasing the number of partition components. The lists of energies from rational values of $\theta$ have far more structure than those from irrational values. In other words, while the lists for rational $\theta$ exhibit finitely many trajectories, the irrational ones appear to display fractal characteristics and tend to become dense in the plane as the number of partition components increases. We will come back to this point later on.

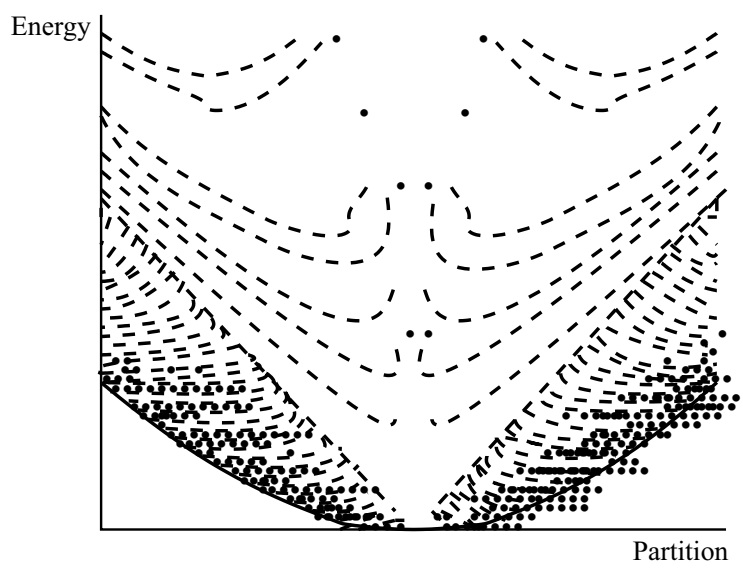

Figure 2: Allowed energies for two-component partitions of the Heisenberg module $\mathcal{E}_{2,2}$ for $\theta=1 / \sqrt{2}$. 
In view of these observations, we may expect that at high energies the degeneracy of states can rise very rapidly, leading to a Hagedorn behaviour of the spectrum. Because the quantum field theory here is semi-classically exact, by a state of the system we shall mean an instanton partition $(p, q)$. We will count the number of solutions of the non-commutative Yang-Mills equations and find appropriate bounds at high energies. These numbers coincide with the volumes of the symmetric orbifold moduli spaces of classical solutions described in [12]. Let $N_{p, q}(E, \theta)$ be the number of submodules of the Heisenberg module $\mathcal{E}_{p, q}$ which are available for making up a partition $\underline{(p, q)}$ that has energy $E=\left(g^{2} A / 2 \pi^{2}\right) S(\underline{(p, q)} ; \theta)$, so that

$$
E=\sum_{k} \frac{q_{k}^{2}}{p_{k}-q_{k} \theta} .
$$

Since the right-hand side of (3.1) is a sum of positive terms, we may infer that $q_{k}^{2} \leq E\left(p_{k}-q_{k} \theta\right)$ for each $k$. But from the constraints (2.10), we also have $0<p_{k}-q_{k} \theta \leq p-q \theta$, and hence the partition component integers are generically bounded on the fixed energy slices as

$$
\left|q_{k}\right| \leq \sqrt{(p-q \theta) E}, \quad q_{k} \theta<p_{k} \leq p-\left(q-q_{k}\right) \theta
$$

for each $k$. They are further constrained by K-theory charge conservation

$$
\sum_{k} p_{k}=p, \quad \sum_{k} q_{k}=q
$$

on the module $\mathcal{E}_{p, q}$.

Let us consider first the case of rational non-commutativity parameter $\theta=n / N$, where $n$ and $N$ are relatively prime positive integers. Submodule dimensions are then all bounded from below by $1 / N$ [12], and by denoting $r_{k}:=N p_{k}-n q_{k}, r:=N p-n q$, the second of the constraints (3.2) reduces to $1 \leq r_{k} \leq r$, while the first sum in (3.3) may be replaced by $\sum_{k} r_{k}=r$. These are just the defining conditions for classical solutions of the Morita equivalent commutative gauge theory with structure group $U(r)$ and they imply that the number of components $m$ of any given partition in this case is bounded from above as $m \leq r$. Because of the first bound in (3.2), there are only finitely many partitions which contribute to a fixed finite energy $E$. The K-theory charges in this instance are completely decoupled and the counting of states is determined by taking the product of the numbers of $q_{k}$ 's and $r_{k}$ 's obeying their respective constraints. The total number of $r_{k}$ 's is the number $\Pi(r)$ of proper unordered partitions of the rank $r$ into natural 
numbers, which can be computed from the generating function [32]

$$
\frac{\mathrm{e}^{\pi \mathrm{i} \tau / 12}}{\eta(\tau)}=1+\sum_{r=1}^{\infty} \Pi(r) \mathrm{e}^{2 \pi \mathrm{i} r \tau},
$$

where

$$
\eta(\tau):=\mathrm{e}^{\pi \mathrm{i} \tau / 12} \prod_{m=1}^{\infty}\left(1-\mathrm{e}^{2 \pi \mathrm{i} m \tau}\right)
$$

is the Dedekind function. For the $q_{k}$ 's, we will strictly bound their number from above by ignoring the topological charge constraint and taking their totality in the range $\left|q_{k}\right| \leq \sqrt{r E / N}$ for each $k=1, \ldots, r$.

In this way, we find that the total number of instanton solutions of energy $E$ satisfies the upper bound

$$
N_{p, q}\left(E, \theta=\frac{n}{N}\right)<\left(2 \sqrt{\frac{r}{N}}\right)^{r} \Pi(r) E^{r / 2}, \quad r=N p-n q .
$$

The important feature of (3.6) is that the corresponding density of states

$$
\rho_{p, q}(E, \theta)=\frac{\partial}{\partial E} N_{p, q}(E, \theta)
$$

has at most a power-like growth $\rho_{p, q}(E, \theta=n / N) \sim E^{\alpha}$ as the energy of the system increases. This is exactly the result that one would anticipate from a gas of non-interacting particles in $r$ dimensions, reflecting again the quantum mechanical nature of the model in this case. The "particles" here are point-like instantons, corresponding to symmetric orbifold singularities, of commutative $U(r)$ Yang-Mills theory on the torus $\mathbb{T}^{2}[12]$.

Now let us analyse the degeneracy of states in the irrational noncommutative gauge theory. The arguments just presented fail in this case, because while in the rational case any submodule dimension is a multiple of the dimension $1 / N$ of the smallest submodule, so that the spacing between submodule dimensions is regular, in the irrational case there is no such lower bound and the spacing between submodule dimensions is irregular. In particular, the topological numbers $\left(p_{k}, q_{k}\right)$ of submodules no longer decouple from each other. We can obtain a crude upper bound on the number of states $N_{p, q}(E, \theta)$ in this instance, starting by restricting the counting to $m$-component partitions. We denote by $\boldsymbol{\Pi}_{m}(p, q ; \theta)$ the subset of partitions $(p, q)$, whose components $\left(p_{k}, q_{k}\right)$ obey the constraints $(2.10)$, with a fixed number $m=|(p, q)|$ of components on the Heisenberg module $\mathcal{E}_{p, q}$ over the algebra $\mathcal{A}=\overline{\mathcal{S}\left(\mathbb{T}_{\theta}^{2}\right)}$. From (3.2), it follows that the topological partition numbers of an element $(p, q) \in \mathbf{\Pi}_{m}(p, q ; \theta)$ lie in the ranges $\left|q_{k}\right| \leq \sqrt{ }(p-q \theta) E$ and $\left|p_{k}\right| \leq|\theta| \sqrt{ }(p-q \theta) E$ for large energies $E$ and for each $k=1, \ldots, m$. Ignoring the constraints (3.3), the degeneracy of states 
$N_{p, q}^{(m)}(E, \theta)$ in $\boldsymbol{\Pi}_{m}(p, q ; \theta)$ can thereby be strictly bounded from above by the hypervolume containing these integers as

$$
N_{p, q}^{(m)}(E, \theta)<\frac{1}{m !}(4|\theta|(p-q \theta) E)^{m},
$$

where we have divided by the permutation symmetry factor $m$ ! to account for the fact that the order of components in the partitions contributing to the vacuum amplitude (2.12) is immaterial. By summing over all possible partition lengths appropriate to the irrational theory, we thereby arrive at the upper bound

$$
N_{p, q}(E, \theta)<\sum_{m=0}^{\infty} N_{p, q}^{(m)}(E, \theta)<\mathrm{e}^{4|\theta|(p-q \theta) E} .
$$

On the other hand, we may argue the form for a lower bound on the number of classical solutions at high energies starting from the explicit expression

$$
N_{p, q}(E, \theta)=\sum_{\substack{\text { partitions } \\ \underline{(p, q)}}} \delta\left(E-\frac{g^{2} A}{2 \pi^{2}} S(\underline{(p, q)} ; \theta)\right)
$$

for the degeneracy of states. To get an idea of the behaviour, let us first consider (3.10) for the "purely non-commutative" Heisenberg modules of K-theory charges $(p, q)=(0, q)$ (these projective modules will play a prominent role in subsequent sections). The counting of solutions in a module of the type $\mathcal{E}_{0, q}$ actually takes care of the general case. A module $\mathcal{E}_{p, q}$ can always be mapped into $\mathcal{E}_{0, \operatorname{gcd}(p, q)}$ via the Morita equivalence generated by the $\mathrm{SL}(2, \mathbb{Z})$ transformation

$$
\left(\begin{array}{c}
0 \\
\operatorname{rank} \mathcal{E}_{p, q}
\end{array}\right)=\left(\begin{array}{c}
\frac{q}{\operatorname{rank} \mathcal{E}_{p, q}}-\frac{p}{\operatorname{rank} \mathcal{E}_{p, q}} \\
c
\end{array}\right)\left(\begin{array}{l}
p \\
q
\end{array}\right),
$$

where $c$ and $d$ are integers obeying the Diophantine relation

$$
c p+d q=\operatorname{gcd}(p, q) .
$$

In particular, every module $\mathcal{E}_{p, q}$ for which $p$ and $q$ are relatively prime is Morita equivalent to $\mathcal{E}_{0,1}$.

By truncating the sum (3.10) to partitions for which $p_{k}=0 \forall k$, we can derive the lower bound

$$
N_{0, q}(E, \theta)>\sum_{q: q_{k} \in \mathbb{N}_{0}} \delta_{|q|, \sum_{k} q_{k}} \delta\left(E-\frac{1}{|\theta|} \sum_{k} q_{k}\right)=\Pi(|\theta| E) .
$$

When $|q|$ is very large, the right-hand side of (3.13) can be thought of as a lower bound at asymptotically high energies, since then $|q|=|\theta| E \rightarrow \infty$. 
The number $\Pi(|q|)$ of unordered partitions of the positive integer $|q|$ into natural numbers has an exact convergent series expansion for large $q$ provided by the Rademacher formula [32]. The first term in this series is the leading term of the asymptotic expansion of $\Pi(|q|)$ at $|q| \rightarrow \infty$ and it yields the Hardy-Ramanujan formula

$$
\Pi(|q|)=\frac{1}{4 \sqrt{3}|q|} \mathrm{e}^{\pi \sqrt{2|q| / 3}}\left[1+\mathrm{O}\left(\frac{\ln |q|}{|q|^{1 / 4}}\right)\right] .
$$

From (3.13) and (3.14), one arrives at the asymptotic energy bound

$$
N_{0, q}(E, \theta)>\frac{\mathrm{e}^{\sqrt{2|\theta| E / 3}}}{4 \sqrt{3}|\theta| E} .
$$

Although only valid in the limit $|q| \rightarrow \infty$, this expression suggests that the corresponding density of states (3.7) for $\theta \in \mathbb{R} \backslash \mathbb{Q}$ has a high energy growth lying between a sub-exponential $\mathrm{e}^{\alpha \sqrt{E}}$ behaviour and an exponential $\mathrm{e}^{\beta E}$ behaviour, in marked contrast to the power growth of the rational case. Note that ordinary two-dimensional Yang-Mills theory at large $N$ exhibits exactly an $\mathrm{e}^{\alpha \sqrt{E}}$ rise in its density of states [33]. The realization of the non-commutative gauge theory as a particular kind of large $N$ limit of commutative gauge theory thereby supports the general validity of (3.15).

With the belief that the lower bound in (3.15) is correct, we can present stronger evidence in favour of an exponential-like behaviour, but with a different power behaviour of the energy in the asymptotic growth. The idea is to embed in a minimal way the previous result in a generic projective module $\mathcal{E}_{p, q}$. The irrationality of $\theta$ allows for the appearence of submodules of $\mathcal{E}_{p, q}$ with arbitrarily small dimension. Let

$$
\varepsilon=p^{\prime}-q^{\prime} \theta
$$

be the dimension of a "small" submodule $\mathcal{E}_{p^{\prime}, q^{\prime}}$. For $\varepsilon \rightarrow 0$, there are always $n$ such submodules appearing in the instanton partition with $n \varepsilon<p-q \theta$. The energy of these configurations is easily estimated to be

$$
E \simeq \frac{n q^{2}}{\varepsilon}
$$

In order to respect the constraint on the total dimension of the module, we have to scale

$$
\varepsilon \simeq \frac{1}{n}
$$

in the limit $n \rightarrow \infty$. Likewise, the smallness of $\varepsilon$ requires that both $p^{\prime}, q^{\prime} \rightarrow$ $\infty$. The defining relation (3.16) can in fact be understood as an approximation procedure for the irrational number $\theta$ by rational numbers as

$$
\frac{p^{\prime}}{q^{\prime}}-\theta=\frac{\varepsilon}{q^{\prime}} .
$$


This equation implies that in constructing the "small" submodules, the integer $q^{\prime}$ scales as $\varepsilon^{-\alpha}$ with $\alpha>0$. Taking into account the scaling of $\varepsilon$ in (3.18), we may then express the growth of $E$ in terms of the number of components $n$ as

$$
E \simeq n^{2+2 \alpha}
$$

The degeneracy of these configuration is given by the number of partitions $\Pi(n)$ and by applying the Hardy-Ramanujan formula (3.14), we have

$$
N_{p, q}(E, \theta)>\frac{a}{E^{1 /(2+2 \alpha)}} \mathrm{e}^{b E^{1 /(4+4 \alpha)}}
$$

for some positive real constants $a$ and $b$. In deriving this bound, we have taken into account the contribution of a very particular set of configurations as $E \rightarrow \infty$, characterized by a peculiar smallness in the submodule decompositions. This is a very crude approximation but, as we have seen, it is sufficient to yield an exponential behaviour for the asymptotic density of states. We have not succeeded in deriving an analog of the lower bound (3.15) for generic topological numbers $(p, q)$, but we believe that at least the characteristic $\mathrm{e}^{\alpha \sqrt{E}}$ behaviour could be obtained by a refined estimate. This former growth is the standard behaviour of the degeneracy of states in ordinary two-dimensional quantum field theory (with finitely many fields and propagating degrees of freedom), while the behaviour $\mathrm{e}^{\beta E}$ in (3.9) is characteristic of the Hagedorn spectrum in string theory. In this sense, the point-like instantons of the commutative (rational) theory become elongated in the irrational non-commutative gauge theory and behave more like the quanta in a system with infinitely many and/or extended degrees of freedom.

Let us now discuss the possible physical consequences of these behaviours. The sum over partitions in (2.12) can be rearranged and written in terms of the instanton density of states as

$$
\begin{aligned}
Z_{p, q}\left(\tilde{g}^{2}, \theta\right) & \left.=\sum_{E>0} \mathrm{e}^{-E / 2 \tilde{g}^{2}} \sum_{\substack{\text { partitions } \\
S(\underline{(p, q) ; \theta)}=\underline{E / 2)}}} W_{p, q} \underline{\left(\underline{(p, q)} ; \tilde{g}^{2}\right.}, \tilde{g}^{2}, \theta\right) \\
& =\int_{0}^{\infty} \mathrm{d} E \rho_{p, q}(E, \theta) \mathrm{e}^{-E / 2 \tilde{g}^{2}} W_{p, q}\left(E ; \tilde{g}^{2}, \theta\right),
\end{aligned}
$$

where $\tilde{g}=g \sqrt{A} / 2 \pi$ is the dimensionless Yang-Mills coupling constant and the function $W_{p, q}$ is the fluctuation determinant in (2.12). In the rational case, whereby the high-energy density of states obeys a power-law increase with $E$, the partition function (3.22) is well-behaved for all $\tilde{g}^{2}$. The lack of Hagedorn behaviour is generic in local quantum field theories, so that if the lower bound of (3.15) represents the true behaviour of the asymptotic density of states in the irrational non-commutative gauge theory, then again there is no instability present in the system. 
On the other hand, the upper bound in (3.9) can lead to an instability if at high energies the exponential growth of the density of states is sufficient to overcome the growth from the quantum fluctuations $W_{p, q}$ in (3.22). If the exponential increase completely dominates over this entropy factor, then the partition function (3.22) will diverge at a critical coupling $\tilde{g}_{\mathrm{c}}^{2}$ which from (3.9) may be estimated to be $\tilde{g}_{\mathrm{c}}^{2} \simeq \frac{1}{8}|\theta|(p-q \theta)$. In this case, the exponential rise in the density of states would lead to a phase transition beyond which the theory develops an instability due to a condensation of instantons in the vacuum. This is somewhat analogous to what happens in ordinary large $N$ $\mathrm{QCD}_{2}$ on the sphere [34-36], although in the non-commutative gauge theory we would not expect the result to rely on a large rank limit, as the effective gauge group is then essentially the infinite unitary group $U(\infty)$ (or more precisely an appropriate completion thereof) [11]. Note that $\tilde{g}_{\mathrm{c}}^{2} \rightarrow \infty$ in the commutative limit $\theta \rightarrow 0$. This would then suggest that the physics beyond the transition point is accurately described by the strong coupling expansion which can be expressed in terms of contributions from the production of virtual electric dipoles in the vacuum [13]. In the commutative case, this would be exactly the regime in which a string representation of the gauge theory is available.

We conclude this section by returning to the possibility of fractal behaviour in the spectrum of non-commutative instantons. We expect that the spacing between energy levels in the spectrum as the energy increases becomes chaotic because there is no longer a finite periodicity in the system, which was represented as the decomposition into classes of spectra in the rational case, as displayed in figure 2 . While the non-commutative gauge theory for irrational $\theta$ is still topological [11], its fundamental degrees of freedom are dipoles with momentum-dependent lengths, and the collection of non-commutative instantons in this case simulates a phase space of extended objects. Note that, due to numerical limitations, the plots of the form in figure 2 are indistinguishable from certain rational plots, reflecting the fact that any irrational number can be approximated by means of an infinite sequence of rational numbers. Only in the irrational case do the trajectories become dense in the plane as the number of partition components increases.

A possible strategy for studying this phenomenon at a more quantitative level is as follows. Let $\mathcal{G}_{m}(p, q ; \theta)$ be the graph of the Yang-Mills action $S: \boldsymbol{\Pi}_{m}(p, q ; \theta) \rightarrow \mathbb{R}_{+}:=(0, \infty)$ defined in (2.11), and restricted to $m$-component partitions. Then

$$
\mathcal{G}_{m}(p, q ; \theta) \subset\left(\mathrm{K}^{0}\left(\mathbb{T}_{\theta}^{2}\right)\right)^{m} \times \mathbb{R}_{+} .
$$

Denoting by

$$
\mathcal{G}(p, q ; \theta):=\bigcup_{m=1}^{\infty} \mathcal{G}_{m}(p, q ; \theta)
$$


the total graph, we can determine the irregularity of the Yang-Mills energy curves by computing the fractal dimension of the set (3.24). If the fractal dimension of the Yang-Mills graph exceeds the topological dimension of the curves making up the graph, we would observe evidence of fractal behaviour in the instanton spectrum. We believe that this phenomenon is a consequence of the denseness of $\mathcal{G}(p, q ; \theta)$ in $\mathbb{R}^{2}$, as suggested by the simplest case depicted in figure 2 , which ought to be a reflection of the denseness of the countable set $K^{0}\left(\mathbb{T}_{\theta}^{2}\right)=\mathbb{Z}+\mathbb{Z} \theta$ on the real line $\mathbb{R}$ [37], and it is reflected in the irregular structure of the curve defined by the Yang-Mills action (2.11) for irrational values of the non-commutativity parameter $\theta$. We will not attempt a rigorous proof here along the lines we have just explained. Multifractal characteristics, of the finite temperature phase diagrams, have also been noted in the supergravity dual of non-commutative Yang-Mills theory in four dimensions [22] and in two-dimensional non-commutative open string theory [38].

The remainder of this paper is devoted to piecing the bits of evidence from this section together. We shall seek a string theory that has the same spectral characteristics as irrational non-commutative Yang-Mills theory. Since the irrational theory may be naturally regarded as a large $N$ commutative gauge theory, there should be a natural string interpretation. While we shall see that this is certainly the case, we find no evidence in favour of a phase transition. In fact, non-commutativity appears to even smoothen out the singularity that is present in the zero area limit of commutative gauge theory on the torus. We will thereby arrive at an open string representation of the non-commutative gauge theory which is valid for all values of the gauge coupling, and is thus in much better shape than its commutative cousin.

\section{Fluxon contributions}

To analyse the possibility of a phase transition as described in the previous section, one would need to acquire a detailed understanding of the entropy function $W_{p, q}\left(E ; \tilde{g}^{2}, \theta\right)$ appearing in (3.22). Unfortunately, this function is rather complicated and in general difficult to deal with analytically. Instead, we shall study a special limit of the gauge theory of Section 2 which maps it onto a gauge theory on the non-commutative plane. Unlike the commutative case, non-commutative gauge theory on $\mathbb{R}^{2}$ generically possesses natural topologically non-trivial gauge field configurations and so even its partition function is non-trivial. The topological configurations which dominate the partition function and survive the limit in this instance themselves independently possess a very natural and direct open string interpretation. This treatment will also naturally connect with the large $N$ limit of commutative 
gauge theory on $\mathbb{T}^{2}$, in which the standard Gross-Taylor string expansion will be exploited in the subsequent sections.

\subsection{Decompactification onto fluxons}

The non-commutative instantons constructed in Section 2 live on a torus $\mathbb{T}^{2}$ of area $A$. In this section, we will analyse what becomes of these configurations in the limit $A \rightarrow \infty$ whereby the non-commutative torus $\mathbb{T}_{\theta}^{2}$ decompactifies onto the non-commutative plane $\mathbb{R}_{\Theta}^{2}$. As we will see, the torus instantons map in a very precise and definite way onto fluxons [26-30], i.e., finite action solutions of the non-commutative Yang-Mills equations on $\mathbb{R}^{2}$ which carry non-zero magnetic flux. In fact, the usual properties of fluxons arise very naturally from their origin as instantons on the non-commutative torus, so that the non-commutative instantons may be regarded as the ancestors of fluxons, or the corresponding topological configurations appropriate to the compactified space.

In performing the decompactification limit, we have to single out those partitions which survive the limit and yield a finite classical action. Once the relevant configurations are identified, we can also evaluate the exact partition function (2.12) in the same limit and give a geometrical interpretation to the instanton expansion presented in [25]. We will first describe in detail the case of a non-commutative torus with rational deformation parameter $\theta$, as it is somewhat more transparent and will be exploited in subsequent sections. The extension to irrational values of $\theta$ is worked out afterwards.

Let us consider the projective module $\mathcal{E}_{p, q}$ with non-commutativitity parameter $\theta=n / N$ where $n, N \in \mathbb{N}_{0}$ are relatively prime. Its dimension (2.2) is given by

$$
\operatorname{dim} \mathcal{E}_{p, q}=\frac{r}{N}, \quad r=N p-n q .
$$

In order to construct the classical solution space, we have to find the general decompositions (2.9) obeying (2.10). The basic observation [12] is that the minimal dimension of an allowed submodule in the present case is $1 / N$. This implies that all dimensions of admissible submodules are quantized in units of $1 / N$, characterized by collections of non-negative integers $\left\{r_{k}\right\}_{k=1}^{r}$ satisfying

$$
\sum_{k=1}^{r} r_{k}=r
$$

with $\operatorname{dim} \mathcal{E}_{p_{k}, q_{k}}=r_{k} / N$. As discussed in the previous section, the number of such collections is the number $\Pi(r)$ of proper unordered partitions of the natural number $r$. 
The next step is to associate a pair of topological numbers $\left(p_{k}, q_{k}\right)$ to each $r_{k}$, in order to completely specify the submodules. The relevant condition is given by the Diophantine equation

$$
N p_{k}-n q_{k}=r_{k}
$$

The general solution of (4.3) is given by

$$
\begin{aligned}
p_{k} & =m_{k} r_{k}+n \widetilde{q}_{k}, \\
q_{k} & =s_{k} r_{k}+N \widetilde{q}_{k},
\end{aligned}
$$

where the integers $m_{k}$ and $s_{k}$ satisfy the $\mathrm{SL}(2, \mathbb{Z})$ constraint

$$
m_{k} N-s_{k} n=1
$$

and $\widetilde{q}_{k} \in \mathbb{Z}$. It is not difficult to prove that the only degrees of freedom remaining are contained in the arbitrary integer $\widetilde{q}_{k}$, as different choices of integer pairs $\left(m_{k}, s_{k}\right)$ obeying the $\mathrm{SL}(2, \mathbb{Z})$ equation (4.5) simply correspond to shifts in $\widetilde{q}_{k}$. In particular, we can fix $\left(m_{k}, s_{k}\right)=(m, s) \forall k$ within a particular submodule decomposition (2.9). The relevant decompositions can therefore be presented as

$$
\mathcal{E}_{p, q}=\bigoplus_{k=1}^{r} \mathcal{E}_{n \widetilde{q}_{k}+m r_{k}, N \widetilde{q}_{k}+s r_{k}},
$$

and the sum rule on $\widetilde{q}_{k}$ is easily derived to be

$$
\sum_{k=1}^{r} \widetilde{q}_{k}=m q-s p .
$$

Thus all classical solutions on the module $\mathcal{E}_{p, q}$, with rational non-commutativity parameter $\theta=n / N$, are specified by the sets of non-negative integers $\left\{r_{k}\right\}_{k=1}^{r}$ and the sets of (generically negative) integers $\left\{\widetilde{q}_{k}\right\}_{k=1}^{r}$ obeying the constraints (4.2) and (4.7).

We are now ready to describe the decompactification limit which maps the quantum gauge theory on the non-commutative torus to a quantum gauge theory on the non-commutative plane. The important point is that, on the non-commutative plane $\mathbb{R}_{\Theta}^{2}$, the relevant parameter $\Theta \in \mathbb{R}$ encoding non-commutativity is dimensionful. On the torus it is given by

$$
\Theta=\frac{A \theta}{2 \pi},
$$

and consequently we have to perform the limit $A \rightarrow \infty$ while keeping fixed the combination (4.8). A natural way to do this in the present case is to 
send $N \rightarrow \infty$ with

$$
A=\frac{2 \pi N \Theta}{n},
$$

while keeping $\Theta$ and $n$ finite. As in the cases of matrix model regularizations [16,39] and the Morita equivalence formulation of non-commutative field theories [25], the large $N$ limit and the infrared limit are correlated, resulting in a double-scaling relation on the classical solutions. The precise geometrical meaning of this double-scaling limit will be described in detail in Section 6.

Let us now identify the configurations which have finite classical action (2.11) in this limit. It is straightforward to see that the relevant sets of non-negative integers $\left\{r_{k}\right\}_{k=1}^{r}$ are given by

$$
\left\{r_{k}\right\}_{k=1}^{r}=\left\{n \ell_{k}\right\}_{k=1}^{\ell} \cup\left\{r^{\prime}\right\} \quad \text { with } \sum_{k=1}^{\ell} \ell_{k}=\ell, \quad r^{\prime}=r-n \ell,
$$

where $\ell$ is a positive integer which is of order 1 in the limit $N \rightarrow \infty$. The associated values of $\widetilde{q}_{k}$ are given by the sets

$$
\left\{\widetilde{q}_{k}\right\}_{k=1}^{r}=\left\{-m \ell_{k}\right\}_{k=1}^{\ell} \cup\left\{q^{\prime}\right\} \quad \text { with } q^{\prime}=m(q+\ell)-s p .
$$

These decompositions correspond to finite action solutions in the set of all classical solutions of the Yang-Mills equations on the projective module $\mathcal{E}_{p, q}$ of fixed topological numbers $(p, q)$. As argued in [25], these are the dominant configurations in the large $N$ limit, with all other contributions being exponentially suppressed in the partition sum (2.12).

With the dominant configurations of topological numbers (4.10) and (4.11), the submodule decompositions (4.6) read

$$
\mathcal{E}_{p, q}=\bigoplus_{k=1}^{\ell} \mathcal{E}_{0,-\ell_{k}} \oplus \mathcal{E}_{p, \ell+q} .
$$

By using (2.11) and (4.9)-(4.11), one finds that the classical solution corresponding to the decomposition (4.12) has action

$$
\begin{aligned}
S\left(\underline{(p, q)} ; \theta=\frac{n}{N}\right) & =\frac{2 \pi^{2} N}{g^{2} A n} \sum_{k=1}^{\ell} \ell_{k}+\frac{2 \pi^{2} N}{g^{2} A} \frac{(\ell+q)^{2}}{N p-n(\ell+q)} \\
& =\frac{\pi \ell}{g^{2} \Theta}+\frac{\pi n}{g^{2} \Theta} \frac{(\ell+q)^{2}}{N p-n(\ell+q)} .
\end{aligned}
$$

In the large $N$ limit, the second term in (4.13) vanishes. Thus only the instantons associated with the first summand of (4.12) contribute to the partition function (2.12). In particular, the full partition function $\mathcal{Z}_{\infty}\left(g^{2}, \Theta\right)$ 
of "physical" gauge theory on $\mathbb{R}_{\Theta}^{2}$, i.e., including a sum over all topological charges, can be obtained from (2.12) in this limit, and it is dominated by the same configurations as in (4.10) and (4.11). Due to a combination of large $N$ and large area suppression, only the instanton partitions corresponding to the first summand of (4.12) yield a non-vanishing contribution, and the sum over partitions for fixed topological numbers $(p, q)$ becomes a sum over $\ell \in \mathbb{N}_{0}$ along with a sum over the $\Pi(\ell)$ proper partitionings in (4.10). One finds

$$
\begin{aligned}
\mathcal{Z}_{\infty}\left(g^{2}, \Theta\right): & =\lim _{A \rightarrow \infty} Z_{p, q}\left(g^{2} A, \theta=\frac{2 \pi \Theta}{A}\right) \\
= & \frac{1}{N \sqrt{g^{2} \Theta}} \sum_{\ell=0}^{\infty}(-1)^{\ell} \mathrm{e}^{-\pi \ell / g^{2} \Theta} \sum_{\substack{\boldsymbol{\nu} \in \mathbb{N}_{0}^{\ell} \\
\sum_{k} k \nu_{k}=\ell}} \prod_{k=1}^{\ell} \frac{(-1)^{\nu_{k}}}{\nu_{k} !} \\
& \times\left(\frac{A^{2}}{2 \pi k^{3} g^{2} \Theta^{3}}\right)^{\nu_{k} / 2},
\end{aligned}
$$

where the (infinite) area $A$ of the non-commutative plane plays the role of an infrared regularization of the quantum field theory.

This calculation suggests that, at the level of classical solutions and the exact semi-classical expansion of the quantum gauge theory on $\mathbb{T}_{\theta}^{2}$, the decompactification limit of a generic projective module $\mathcal{E}_{p, q}$ receives contributions only from submodules of the type $\mathcal{E}_{0,-\ell_{k}}$, with the dimension positivity constraints $p_{k}-q_{k} \theta>0$ in (2.10) requiring $\ell_{k} \geq 0$ for $\theta>0$ (for $\theta<0$, one would instead find $\mathcal{E}_{0, \ell_{k}}$ with $\ell_{k} \leq 0$ ). The classical action is proportional to $\ell=\sum_{k} \ell_{k}$ with $\ell$ an arbitrary integer. No information is retained of the original geometric data of the gauge theory on the non-commutative torus, i.e., its topological numbers $(p, q)$. This is simply the action of the $|\ell|$-fluxon solution of $U(1)$ Yang-Mills theory on the non-commutative plane [27,30].

It is important to note here that the zero action configurations in the decompactification limit are suppressed from the partition function by area factors coming from the quantum fluctuation determinants. It is also remarkable that, starting from any $(p, q)$, the resulting quantum theory on the non-commutative plane naturally includes contributions from all topological sectors labelled by the fluxon charge $\ell$. The sum over $\ell \in \mathbb{N}_{0}$, which labels the partitions surviving the decompactification limit, arises in the vacuum amplitude (2.12) over the module $\mathcal{E}_{p, q}$ because of the original sum over partitions. In this sense, the quantum gauge theory in the limit is "universal", in that it is independent of the particular Heisenberg module on which it is defined. Moreover, the contributions from any finite action fluxon configuration are identical, as the spurious dependences (e.g., on $\ell+q$ 
in (4.12)) are washed out in the limit $\theta \rightarrow 0$ in every topological sector of the non-commutative gauge theory.

The same expressions were obtained by different means in [25]. There a $U(1)$ non-commutative gauge theory on a torus of rational non-commutativity parameter $1 / N$ was considered and Morita equivalence was used to map the system onto a commutative one. The decompactification limit was taken as in (4.9), resulting in a peculiar large $N$ limit on the partition function of (commutative) $S U(N) / \mathbb{Z}_{N}$ Yang-Mills theory on $\mathbb{T}^{2}$, which can be computed exactly by standard techniques $[40,41]$. In that case, the size of the Morita equivalent torus vanishes as $1 / N$, the 't Hooft limit of the Yang-Mills coupling constant must be taken, and the topological charge remains finite in the limit. In the original $\mathrm{SU}(N) / \mathbb{Z}_{N}$ theory, there are exactly $N$ topological sectors characterizing the inequivalent principal $\mathrm{SU}(N) / \mathbb{Z}_{N}$ gauge bundles over $\mathbb{T}^{2}[42]$. The fluxon sum then appears as the sum over configurations surviving the large $N$ limit in the $\mathrm{SU}(N) / \mathbb{Z}_{N}$ partition function for a fixed topological sector in $\mathbb{Z}_{N}$. This technique will be exploited later on within another, related context.

Finally, it is straightforward to see that precisely the same fluxon configurations are recovered in the case of irrational deformation parameter $\theta$. In the limit $A \rightarrow \infty$ with the combination (4.8) held finite, the field strengths (2.4) vanish for $p \neq 0$, as do the fluctuation factors in (2.12). Thus in the decompactification limit, only the partitions associated to the Heisenberg submodules $\mathcal{E}_{0,-\ell_{k}}$ have finite action and survive in the quantum field theory. As in the rational case, a generic instanton has vanishing classical action in the limit. The only constant curvature connections, with non-trivial action in the decompactification limit, derive from modules of the type $\mathcal{E}_{0,-\ell_{k}}$.

\subsection{Decompactification of instanton configurations}

While the analysis of the previous section provides circumstantial evidence that the instantons on non-commutative $\mathbb{T}^{2}$ decompactify onto fluxons on non-commutative $\mathbb{R}^{2}$, we can actually go further and obtain a one-to-one correspondence between the two sets of non-trivial gauge field configurations in the limit. Let us assume for definiteness that $\theta>0$. Recall that any projective module over the algebra $\mathcal{A}_{\infty}=\mathcal{S}\left(\mathbb{R}_{\Theta}^{2}\right)$ of Schwartz functions on the non-commutative plane is of the form $\mathcal{F}^{\ell} \oplus\left(\mathcal{A}_{\infty}\right)^{m}[7]$, and is thereby characterized by two non-negative integers $\ell$ and $m$ which are, respectively, the magnetic flux quantum number and gauge group rank of the corresponding gauge bundle over $\mathbb{R}_{\Theta}^{2}$. It follows that the K-theory group of the non-commutative plane is $K^{0}\left(\mathbb{R}_{\Theta}^{2}\right)=\mathbb{Z}^{2}$. However, the positive cone $\mathbb{N}^{2}$ 
is rather different than that of $\mathrm{K}^{0}\left(\mathbb{T}_{\theta}^{2}\right)$ and as a consequence one cannot realize modules with negative magnetic flux. There is no natural way to distinguish between the deformations corresponding to $\Theta$ and $-\Theta$ on the plane, as all deformations are Morita equivalent in this case because the Heisenberg commutation relations have a unique irreducible representation, the Schrödinger representation of quantum mechanics, irrespective of the value of $\Theta \neq 0$ (this is the Stone-von Neumann theorem). In the context of fluxon solutions, non-commutativity breaks charge conjugation symmetry and one cannot simply produce anti-vortices from vortices via a change of orientation on $\mathbb{R}^{2}$. Thus any theory involving fluxons will be chiral $[7,8,30]$. In the following, we will consider the rank 1 case $m=1$.

Let us now consider the contributing projective module decompositions (4.12) over the non-commutative torus in the decompactification limit. Generally, from (2.4)-(2.6) it follows that there is a natural isomorphism $\mathcal{E}_{k p, k q} \cong$ $\left(\mathcal{E}_{p, q}\right)^{k}$, and so it suffices to consider the total submodule

$$
\mathcal{E}_{0,-\ell}=\bigoplus_{k=1}^{\ell} \mathcal{E}_{0,-\ell_{k}}=\mathcal{F} \otimes \mathbb{C}^{\ell} \cong \mathcal{F}^{\ell}
$$

for $\ell>0$. This isomorphism is a consequence of the fact that, for $p=0$, the Weyl-'t Hooft algebra (2.6) reduces simply to the requirement that $\Gamma_{1} \Gamma_{2}=\Gamma_{2} \Gamma_{1}$ which may be solved generically (up to unitary isomorphism) by arbitrary $\ell \times \ell$ diagonal unitary matrices

$$
\Gamma_{i}=\mathrm{e}^{-2 \pi \mathrm{i} \gamma_{i} / \sqrt{A}}:=\left(\begin{array}{lll}
\mathrm{e}^{-2 \pi \mathrm{i} \gamma_{i}^{(0)} / \sqrt{A}} & & \\
& \ddots & \\
& & \mathrm{e}^{-2 \pi \mathrm{i} \gamma_{i}^{(\ell-1)} / \sqrt{A}}
\end{array}\right)
$$

with $\gamma_{i}^{(k)} \in \mathbb{R}, i=1,2, k=0,1, \ldots, \ell-1$. Using (2.4) and (4.8), one identifies the field strength of the global instanton of the module (4.15) in the decompactification limit as

$$
F_{0,-\ell}=\frac{\mathrm{i}}{\Theta} \mathrm{B}_{\theta}^{\ell}
$$

where we have explicitly represented the K-theory class of $\mathcal{E}_{0,-\ell}$ through the Boca projection $\mathrm{B}_{\theta}^{\ell}=\mathrm{P}_{0,-\ell}$ on the non-commutative torus with $\operatorname{Tr} \mathrm{B}_{\theta}^{\ell}=$ $\ell \theta[43-45]$. The trace of the projector $\mathrm{P}_{\ell}^{\prime}=A \mathrm{~B}_{\theta}^{\ell}$ over $\mathcal{E}_{0,-\ell}$ is

$$
\operatorname{Tr} \mathrm{P}_{\ell}^{\prime}=2 \pi \Theta \ell
$$


and it is possible to show $[44,45]$ that its decompactification limit yields the canonical rank $\ell$ projector

$$
\frac{1}{2 \pi \Theta} \mathrm{P}_{\ell}^{\prime}=\mathrm{P}_{\ell}:=\sum_{k=0}^{\ell-1}|k\rangle\langle k|
$$

on the Fock module

$$
\mathcal{F}=\bigoplus_{k=0}^{\infty} \mathbb{C} \cdot|k\rangle
$$

over the non-commutative plane $\mathbb{R}_{\Theta}^{2}$.

We now identify the classical gauge field configurations in the limit. In (2.7), we wish to make an identification of the form $U_{i} \sim \mathrm{e}^{2 \pi \mathrm{i} x_{i} / \sqrt{A}}$, with $x_{i}, i=1,2$ the coordinate generators of $\mathbb{R}_{\Theta}^{2}$ obeying the Heisenberg algebra

$$
\left[x_{1}, x_{2}\right]=\mathrm{i} \Theta .
$$

However, one needs to be careful in making a naive equality, because the $x_{i}$ are operators on the trivial rank 1 module $\mathcal{A}_{\infty}$, not on the Heisenberg module (4.15) on which

$$
U_{i}=\mathrm{e}^{-(2 \pi / \sqrt{A})\left(\Theta \nabla_{i} \otimes \mathbb{1}_{\ell}+\mathrm{i} \mathbb{1} \otimes \boldsymbol{\gamma}_{i}\right)}
$$

are represented. In fact, we can identify the Heisenberg module (4.15) as a proper submodule of $\mathcal{A}_{\infty}$ by using the fact that the rank 1 free module can be decomposed as

$$
\mathcal{A}_{\infty}=\bigoplus_{k=0}^{\infty}|k\rangle\langle k| \cdot \mathcal{A}_{\infty},
$$

where the operator $|k\rangle\langle k|, k \in \mathbb{N}_{0}$ is the orthogonal projection onto the onedimensional subspace spanned by the vector $|k\rangle \in \mathcal{F}$, and here we regard the algebra $\mathcal{A}_{\infty}$ in its irreducible representation on the Fock space (4.20) given by functionals of the standard creation and annihilation operators. For each $k \in \mathbb{N}_{0}$, there is an isomorphism $|k\rangle\langle k| \cdot \mathcal{A}_{\infty} \cong \mathcal{F}$ given by the mapping $|k\rangle\langle k|\cdot f \mapsto f| k\rangle \in \mathcal{F}$ for $f \in \mathcal{A}_{\infty}$. In particular, using this isomorphism we have

$$
\mathcal{E}_{0,-\ell} \cong \mathrm{P}_{\ell} \cdot \mathcal{A}_{\infty}
$$

where $\mathrm{P}_{\ell}$ is the orthogonal projection (4.19) on $\mathcal{F} \rightarrow \mathbb{C}^{\ell}$. Equation (4.24) is just the definition of (2.1) in the decompactification limit of the torus.

Making the desired identification in the unitary generators $U_{i}$ requires that all operators be defined on a common domain. We circumvent this 
difficulty by trivially embedding the pertinent operators into the module $\mathcal{E}_{0,-\ell} \oplus \mathcal{A}_{\infty}$ as

$$
\begin{aligned}
& \widehat{\nabla}_{i}:=\left(\nabla_{i} \otimes \mathbb{1}_{\ell}\right) \oplus 0, \\
& \widehat{\gamma}_{i}:=\left(\mathbb{1} \otimes \boldsymbol{\gamma}_{i}\right) \oplus 0, \\
& \widehat{x}_{i}:=\mathbf{0}_{\ell} \oplus x_{i} .
\end{aligned}
$$

We then represent all these operators on the free module $\mathcal{A}_{\infty}$ by finding their images under a unitary isomorphism of separable Hilbert spaces

$$
\Omega_{\ell}: \mathcal{E}_{0,-\ell} \oplus \mathcal{A}_{\infty} \stackrel{\approx}{\longrightarrow} \mathcal{A}_{\infty} .
$$

That such an isomorphism between $\mathcal{A}_{\infty}$-modules exists follows from (4.24) and is just the usual statement that any gauge bundle over the plane is trivial. In this way, we can then relate the torus generators (4.22), regarded now as operators on $\mathcal{A}_{\infty}$, to exponentials of the plane generators obeying (4.21) through

$$
U_{i}=\Omega_{\ell} \mathrm{e}^{-(2 \pi / \sqrt{A})\left(\Theta \widehat{\nabla}_{i}+\mathrm{i} \widehat{\gamma}_{i}\right)} \Omega_{\ell}^{-1}:=\Omega_{\ell} \mathrm{e}^{2 \pi \mathrm{i} \widehat{x}_{i} / \sqrt{A}} \Omega_{\ell}^{-1} .
$$

The mapping $\Omega_{\ell}$ can be constructed explicitly by introducing the standard shift operator

$$
\mathrm{S}_{\ell}=\left(\mathrm{S}_{1}\right)^{\ell}:=\sum_{k=0}^{\infty}|k+\ell\rangle\langle k|
$$

on the Fock module (4.20), which is a partial isometry on $\mathcal{F}$ obeying

$$
\mathrm{S}_{\ell}^{\dagger} \mathrm{S}_{\ell}=\mathbb{1}, \quad \mathrm{S}_{\ell} \mathrm{S}_{\ell}^{\dagger}=\mathbb{1}-\mathrm{P}_{\ell}
$$

In particular, the operator $S_{\ell}$ is a unitary isomorphism on the orthogonal complement in $\mathcal{F}$ to a finite-dimensional cokernel, i.e., $\operatorname{ker} S_{\ell}=\{0\}$ and $\operatorname{ker} \mathrm{S}_{\ell}^{\dagger}=\operatorname{im} \mathrm{P}_{\ell} \cong \mathbb{C}^{\ell}$. This implies that the submodule $\mathrm{S}_{\ell} \cdot \mathcal{A}_{\infty}$ is the orthogonal complement in $\mathcal{A}_{\infty}$ of the submodule (4.24) and $\mathrm{S}_{\ell}$ can thereby be used to construct the isomorphism we seek. We thus define (4.26) as

$$
\Omega_{\ell}\left(\sum_{k=0}^{\ell-1} f|k\rangle \oplus f^{\prime}\right):=\mathrm{P}_{\ell} \cdot f+\mathrm{S}_{\ell} \cdot f^{\prime}
$$

with inverse given by

$$
\Omega_{\ell}^{-1}(f)=\sum_{k=0}^{\ell-1} f|k\rangle \oplus \mathrm{S}_{\ell}^{\dagger} \cdot f,
$$

where $f, f^{\prime} \in \mathcal{A}_{\infty}$. 
It is straightforward to now work out the images on $\mathcal{A}_{\infty} \rightarrow \mathcal{A}_{\infty}$ of the operators (4.25) under this isomorphism and one finds

$$
\begin{aligned}
& \Omega_{\ell} \widehat{x}_{i} \Omega_{\ell}^{-1}(f)=\mathrm{S}_{\ell} x_{i} \mathrm{~S}_{\ell}^{\dagger} \cdot f, \\
& \Omega_{\ell} \widehat{\gamma}_{i} \Omega_{\ell}^{-1}(f)=\sum_{k=0}^{\ell-1} \gamma_{i}^{(k)}|k\rangle\langle k| \cdot f .
\end{aligned}
$$

From (4.27) and (4.32), we arrive finally at an expression for the gauge connection in the decompactification limit as an operator on the free module $\mathcal{A}_{\infty}$ given by

$$
D_{i}:=\mathrm{i} \Theta \Omega_{\ell} \widehat{\nabla}_{i} \Omega_{\ell}^{-1}=\mathrm{S}_{\ell} x_{i} \mathrm{~S}_{\ell}^{\dagger}+\sum_{k=0}^{\ell-1} \gamma_{i}^{(k)}|k\rangle\langle k| .
$$

The operator (4.33) is precisely the gauge field configuration of the $\ell$-fluxon solution of $U(1)$ non-commutative Yang-Mills theory on the Fock module (4.15) over $\mathbb{R}_{\Theta}^{2}[7,8,27,29,30]$.

\subsection{Properties of fluxons}

The realization of fluxons in this way by their pre-images as instantons on the non-commutative torus immediately implies many of the peculiar features that these vortex solutions exhibit. These properties are not so unusual in the parent theory where they are completely transparent, as they follow from some of the basic properties of Heisenberg modules. Because of the richer topology, there are of course many more non-commutative instantons on $\mathbb{T}^{2}$ than there are non-commutative vortices on $\mathbb{R}^{2}$, but when the torus is decompactified only a subset of the instantons survive and become localized vortex solutions on the non-commutative plane. Note that this is a quantum effect, as it arises from the suppression of all other instanton modes in the fluctuation determinants of the vacuum amplitude (2.12).

Let us now summarize some of the novel properties that the fluxons naturally inherit in this way:

1. Fluxons only exist with one sign of the magnetic charge $\operatorname{sgn} \ell=\operatorname{sgn} \theta$. This is a consequence of the positivity of the Murray-von Neumann dimension (2.2) of the module $\mathcal{E}_{0,-\ell}$.

2. The quadratic dependence of the action (2.11) on the instanton charge becomes linear when $p_{k}=0$, explaining the linear growth of the soliton mass with the topological charge $\ell$.

3 . The $2 \ell$-dimensional moduli space of solutions for magnetic charge $\ell>0$ corresponding to the separations of the vortices comes from the trivial 
representation (4.16) on $\mathbb{C}^{\ell}$ of the generators (2.8) for the module $\mathcal{E}_{0,-\ell}$. Thus the intrinsic structure of Heisenberg modules induces the fluxon moduli space.

4. By using (4.17) and (4.32), the field strength of the gauge field (4.33) is easily worked out to be

$$
\frac{\mathrm{i} A}{2 \pi \Theta^{2}}\left[D_{1}, D_{2}\right]=-\frac{\mathrm{i} A}{2 \pi \Theta} \Omega_{\ell} \widehat{F}_{0,-\ell} \Omega_{\ell}^{-1}=\mathrm{P}_{\ell}
$$

with $\widehat{F}_{0,-\ell}=\left[\widehat{\nabla}_{1}, \widehat{\nabla}_{2}\right]$, implying that the configuration has quantized magnetic charge $\operatorname{Tr} \mathrm{P}_{\ell}=\ell$. By construction on $\mathbb{T}_{\theta}^{2}$, the field strength (4.17) a priori has the usual non-commutative vortex form in terms of a projection operator on a Fock module $[7,8]$, coming from its origin in terms of a constant curvature connection over the non-commutative torus.

5. From (4.34), it follows that the energy of the configuration of vortices is independent of their position moduli $\gamma_{i}^{(k)} \in \mathbb{R}$. As a consequence, the semi-classical expansion of the quantum gauge theory about the fluxons diverges [30] and must be regulated by an infrared cutoff $A$, the finite size of the torus $\mathbb{T}^{2}$, as in (4.14). The exact fluctuation spectrum around each fluxon is given by the pre-exponential factors of the exact path integral (4.14) of gauge theory on the non-commutative plane. It contains, in particular, the correct moduli dependence $A^{\nu_{1}+\cdots+\nu_{\ell}}$ of the collections of $\nu_{k}$ elementary constituent vortices of charge $k$ inside the $\ell$-fluxon solution, along with the permutation symmetry factors $1 / \nu_{k}$ ! appropriate to the identical vortices of equal charge inside each soliton [25].

6. All fluxons of charges $\ell \in \mathbb{N}_{0}$ originate from the same Heisenberg module $\mathcal{E}_{p, q}$ over the non-commutative torus, with fixed topological numbers $(p, q) \in \mathrm{K}^{0}\left(\mathbb{T}_{\theta}^{2}\right)$. This fact has two important implications. First of all, although the gauge connection (4.33) is constructed from the globally minimizing constant curvature connection on the Heisenberg module (4.15), it originates from the submodule decompositions (4.12) which generically correspond to unstable extrema of the gauge theory on $\mathbb{T}_{\theta}^{2}$. This naturally explains the instability of the fluxon solutions on the noncommutative plane $[29,30]$. Second, the fluxon charge $\ell$ is not a conserved quantum number [8]. Because of their metastability, it is possible for a vortex solution to interpolate between different topological sectors characterized by total flux. This physical statement is in no contradiction with the conventional definitions, provided we consider it from the point of view of the instanton ancestors.

Thus the torus instanton origin of fluxons described above naturally explains their known properties in very simple settings, and moreover implies new features of them. On the other hand, the $\ell$-fluxon solution (4.33) can 
be interpreted [29] as a non-BPS configuration of $\ell$ Euclidean $\mathrm{D}(-1)$-branes at positions $\left(\gamma_{1}^{(k)}, \gamma_{2}^{(k)}\right), k=0,1, \ldots, \ell-1$ on a Euclidean D1-brane, in the presence of a background $B$-field in the Seiberg-Witten decoupling limit [10]. Its fluctuation spectrum contains a tachyon and at the endpoint of tachyon condensation the D-instantons are completely dissolved in the D-string. It is natural to now examine if this string origin of fluxons can be understood through the stringy properties of the torus instantons that we unveiled in the previous section. We will begin attacking this problem in the next section. Note that the charge $\ell$ of a fluxon is proportional to its energy $E$, and for each $\ell$ there are $\Pi(\ell)$ states in (4.14) which contribute at fixed energy $E$. It then follows from the Hardy-Ramanujan formula (3.14) that the asymptotic density of states has the characteristic sub-exponential behaviour $\mathrm{e}^{\alpha \sqrt{E}}$ of quantum field theory. This strongly suggests that, despite their instability, the fluxon contributions do not drive the system to a phase transition. In Section 7 , we will show that this is indeed the case.

\subsection{The fluxon partition function}

For later use, let us now explicitly sum the fluxon expansion (4.14). For this, we unravel the constraint on the partitions $\boldsymbol{\nu}$ via a contour integral representation for the Kronecker delta function given by

$$
\delta_{m n}=\oint_{\mathcal{C}_{0}} \frac{\mathrm{d} z}{2 \pi \mathrm{i} z} z^{m-n}
$$

where $m, n \in \mathbb{Z}$ and the closed contour $\mathcal{C}_{0}$ encircles the origin $z=0$ of the complex $z$-plane with counterclockwise orientation. At this stage $\mathcal{C}_{0}$ is arbitrary, but later on we will have to choose it carefully for convergence reasons. We may thereby write the amplitude (4.14) as a sum over unconstrained integers as

$$
\begin{aligned}
\mathcal{Z}_{\infty}\left(g^{2}, \Theta\right)= & \sum_{\ell=0}^{\infty}(-1)^{\ell} \mathrm{e}^{-\pi \ell / g^{2} \Theta} \sum_{\nu \in \mathbb{N}_{0}^{\ell}} \prod_{k=1}^{\ell} \frac{(-1)^{\nu_{k}}}{\nu_{k} !}\left(\frac{A^{2}}{2 \pi k^{3} g^{2} \Theta^{3}}\right)^{\nu_{k} / 2} \\
& \times \oint_{\mathcal{C}_{0}} \frac{\mathrm{d} z}{2 \pi \mathrm{i} z^{\ell+1}} z^{\sum_{l} l \nu_{l}}
\end{aligned}
$$

where we have discarded the overall multiplicative constant. The sums can now be done explicitly thanks to the contour integration, and one finds

$$
\mathcal{Z}_{\infty}\left(g^{2}, \Theta\right)=\oint_{\mathcal{C}_{0}} \frac{\mathrm{d} z}{2 \pi \mathrm{i}} \frac{1}{z+\mathrm{e}^{-\pi / g^{2} \Theta}} \exp \left[-\frac{A}{\sqrt{2 \pi g^{2} \Theta^{3}}} \mathrm{Li}_{3 / 2}(-z)\right],
$$


where

$$
\operatorname{Li}_{\alpha}(z):=\sum_{k=1}^{\infty} \frac{z^{k}}{k^{\alpha}}
$$

is the Jonquière polylogarithm function of index $\alpha \in \mathbb{C}$. We will therefore restrict the contour of integration $\mathcal{C}_{0}$ in (4.37) to lie in the open annulus $\mathrm{e}^{-\pi / g^{2} \Theta}<|z|<1$ in order to ensure convergence of the series (4.38) and to catch the pole at $z=-\mathrm{e}^{-\pi / g^{2} \Theta}$. This constraint can be relaxed somewhat via an appropriate analytic continuation which is described in Appendix A.

The residue theorem now implies our final expression

$$
\mathcal{Z}_{\infty}\left(g^{2}, \Theta\right)=\exp \left[-\frac{A}{\sqrt{2 \pi g^{2} \Theta^{3}}} \operatorname{Li}_{3 / 2}\left(\mathrm{e}^{-\pi / g^{2} \Theta}\right)\right]
$$

for the partition function of quantum gauge theory on the non-commutative plane [25]. This exact extensive form of the vacuum amplitude is typical of a dilute instanton gas approximation. It is consistent with the fact that fluxons are non-interacting solitons.

\section{$5 \quad$ Instanton string expansions}

In the previous section we found that fluxons on the non-commutative plane are dynamically induced by the topological configurations on the noncommutative torus. As the non-commutative gauge theory can be obtained from a commutative $\mathrm{SU}(N) / \mathbb{Z}_{N}$ gauge theory through a large $N$ doublescaling limit [25], it is natural to explore the problem of obtaining the open string interpretation of fluxons through the conventional closed string expansion of ordinary Yang-Mills theory in two spacetime dimensions. However, this string representation is based on the strong-coupling expansion of the commutative gauge theory. As we mentioned earlier, while such an expansion is available for the gauge theory on the non-commutative torus [13], it is rather complicated and does not straightforwardly admit nice algebraic or geometric characterizations. We must therefore try to extract the string representation of two-dimensional non-commutative Yang-Mills theory directly from its weak-coupling expansion. To understand this point, in this section we will revisit the Gross-Taylor string expansion of commutative $U(N)$ Yang-Mills theory on the torus $\mathbb{T}^{2}$ and analyse how it manifests itself in the instanton series. 


\subsection{Gross-Taylor series on the torus}

The chiral partition function of ordinary $U(N)$ Yang-Mills theory on the torus $\mathbb{T}^{2}$ is a variant of the usual Migdal strong-coupling expansion [40,41] given by

$$
Z_{U(N)}^{+}(\lambda)=\sum_{R \in \operatorname{Rep}^{+}(U(N))} \mathrm{e}^{-(\lambda / N) C_{2}(R)},
$$

where

$$
\lambda=\frac{g^{2} A N}{2}
$$

is the dimensionless 't Hooft coupling constant and $C_{2}(R)$ is the quadratic Casimir eigenvalue in the irreducible representation $R=R(Y)$ of the gauge group $U(N)$. The restriction to chiral representations means considering Young tableaux $Y$ with positive number of boxes and dropping the constraint that the number of rows be less than the rank $N$. In so doing, one essentially assumes that only representations with small numbers of boxes (compared to $N$ ) are relevant in the large $N$ limit, the others being exponentially suppressed in $N$ [3]. More generally, a class of representations with box numbers of order $N$, whose contribution is not exponentially damped, can be found and used to construct the anti-chiral sector [4]. A non-chiral coupled expansion, incorporating both contributions, has been presented in $[4,5]$ for the case of an $\mathrm{SU}(N)$ gauge group and is widely accepted as the complete large $N$ description of the commutative gauge theory.

For a Young diagram $Y \in \mathcal{Y}_{n}$ of $n$ boxes, the quadratic Casimir can be written as

$$
C_{2}(R(Y))=N n+\widetilde{C}_{2}(R(Y)),
$$

where $\widetilde{C}_{2}(R(Y))$ is of order 1 in the large $N$ limit. The partition function (5.1) then admits the asymptotic $1 / N$ expansion

$$
Z_{U(N)}^{+}(\lambda)=\sum_{n=0}^{\infty} \sum_{Y \in \mathcal{Y}_{n}} \mathrm{e}^{-n \lambda} \sum_{m=0}^{\infty} \frac{\left[-\lambda \widetilde{C}_{2}(R(Y))\right]^{m}}{m !} \frac{1}{N^{m}} .
$$

The irreducible representations $R(Y)$ are labelled by non-increasing partitions $\infty>n_{1} \geq n_{2} \geq \cdots \geq n_{n} \geq 0, \sum_{k} n_{k}=n$ corresponding to the conjugacy classes of the symmetric group $S_{n}$ and specifying the lengths of rows in the Young diagram $Y \in \mathcal{Y}_{n}$ with

$$
\widetilde{C}_{2}(R(Y))=\sum_{k=1}^{n} n_{k}\left(n_{k}+1-2 k\right) .
$$

This quantity is the central character of the corresponding irreducible representation of $S_{n}$. 
In this way, the expansion (5.4) acquires the form [4]

$$
Z_{U(N)}^{+}(\lambda)=1+\sum_{h=1}^{\infty} \frac{1}{N^{2 h-2}} \sum_{n=1}^{\infty} \mathrm{e}^{-n \lambda} \sum_{m=1}^{\infty} \omega_{h}^{m, n}(2 \lambda)^{m},
$$

where the coefficients $\omega_{h}^{m, n} \in \mathbb{N}_{0}$ give the number of (topological classes of) $n$-sheeted holomorphic covering maps without folds to the torus $\mathbb{T}^{2}$. They count the number of (disconnected) maps from a closed oriented Riemann surface of genus $h$ to the torus with winding number $n$ and $m$ simple branch points. The expansion (5.6) contains a Nambu-Goto factor $\mathrm{e}^{-n \lambda}$ as well as a volume factor $\lambda^{m}$ from the moduli space integration over the positions of the branch points. It is important to note that this is only the chiral expansion of the gauge theory. The full $\mathrm{SU}(N)$ partition function also couples a sector of anti-holomorphic maps, along with singular geometrical contributions such as collapsed handles and infinitesimal tubes connecting the sheets of the holomorphic covers with those of the anti-holomorphic covers. Such singularities come from the boundary of moduli space where worldsheet handles degenerate to a point in the spacetime $\mathbb{T}^{2}$, and they complicate the form of $\omega_{h}^{m, n}$. However, at the present level of the chiral $U(N)$ theory, these natural numbers are of the form $\omega_{h}^{m, n}=\omega_{h}^{n} \delta_{m, 2 h-2}$ by the Riemann-Hurwitz theorem, and the explicit summation over Young diagrams in (5.4) yields

$$
\begin{aligned}
\omega_{h}^{n}= & \sum_{k=1}^{n} \sum_{\substack{n \in \mathbb{N}^{k}, \sum_{l} n_{l}=n \\
n_{1} \geq n_{2} \geq \cdots \geq n_{k}}}\left[\sum_{l=1}^{k} \frac{\left(n_{l}+k-l\right)\left(n_{l}+k-l-1\right)}{2}\right. \\
& \left.\times \prod_{l^{\prime} \neq l}\left(\frac{n_{l}-n_{l^{\prime}}+l^{\prime}-l-2}{n_{l}-n_{l^{\prime}}+l^{\prime}-l}\right)\right]^{2 h-2},
\end{aligned}
$$

which is in agreement with earlier results from combinatorial group theory $[46,47]$.

The free energy $F_{U(N)}^{+}(\lambda)=\ln Z_{U(N)}^{+}(\lambda)$ can be written as

$$
F_{U(N)}^{+}(\lambda)=\sum_{h=1}^{\infty} \frac{1}{N^{2 h-2}} F_{h}^{+}(\lambda)
$$

where

$$
F_{h}^{+}(\lambda)=(2 \lambda)^{2 h-2} \sum_{n=1}^{\infty} \sigma_{h}^{n} \mathrm{e}^{-n \lambda}
$$

The non-negative integers

$$
\sigma_{h}^{n}=\sum_{k=1}^{n} \frac{(-1)^{k}}{k} \sum_{\substack{n \in \mathbb{N}^{k} \\ \sum_{l} n_{l}=n}} \sum_{\substack{\boldsymbol{h} \in \mathbb{N}^{k} \\ \sum_{l} h_{l}=h}} \omega_{h_{1}}^{n_{1}} \cdots \omega_{h_{k}}^{n_{k}}
$$


are called simple Hurwitz numbers and they count the connected (irreducible) branched covering maps to $\mathbb{T}^{2}$. In other words, the strong coupling expansion for the chiral free energy of $U(N)$ gauge theory on $\mathbb{T}^{2}$ in the 't Hooft limit is the generating function for the Hurwitz numbers, and the expansion (5.8) can be identified as the partition function of a string theory with torus target space. The coupling and tension are given by

$$
g_{s}=\frac{1}{N}, \quad T=\frac{1}{2 \pi \alpha^{\prime}}=\frac{\lambda}{A} .
$$

There are two important and non-trivial features in this context displayed by the expansion (5.8) of the gauge theory free energy. First of all, only even powers $N^{2-2 h}$ of the rank $N$ appear weighted by the Euler characteristic of the covering surface and this is essential for a closed string interpretation. Second, there is no term of order $N^{2}$ in the expansion, consistent with the fact that there are no unfolded covers of a torus by a sphere.

One of the most interesting properties of the $\mathrm{QCD}_{2}$ string partition function on $\mathbb{T}^{2}$ is that the contributions (5.9) are quasi-modular forms on the elliptic curve whose Kähler class is dual to the modulus

$$
\tau=-\frac{\lambda}{2 \pi \mathrm{i}},
$$

with $F_{h}^{+}(\tau), h \geq 2$ of weight $6 h-6$ under the full modular group $\operatorname{PSL}(2, \mathbb{Z})$ [48-50]. Quasi-modular forms are polynomials in the basic holomorphic Eisenstein series $E_{2}(\tau), E_{4}(\tau)$, and $E_{6}(\tau)$, where

$$
E_{k}(\tau):=1-\frac{2 k}{B_{k}} \sum_{n=1}^{\infty} \sum_{d \mid n} d^{k-1} \mathrm{e}^{2 \pi \mathrm{i} n \tau}, \quad k \in 2 \mathbb{N},
$$

and $B_{k} \in \mathbb{Q}$ is the $k$ th Bernoulli number. The Eisenstein series $E_{4}(\tau)$ and $E_{6}(\tau)$ are, respectively, modular forms of weights 4 and 6 ,

$$
E_{k}\left(\frac{a \tau+b}{c \tau+d}\right)=(c \tau+d)^{k} E_{k}(\tau), \quad k=4,6, \ldots,
$$

where

$$
\left(\begin{array}{ll}
a & b \\
c & d
\end{array}\right) \in \operatorname{PSL}(2, \mathbb{Z})
$$

and they generate the ring $\mathcal{M}^{0}$ of all modular forms. On the other hand, the form $E_{2}(\tau)$ is not quite of weight 2 but has a modular anomaly given by

$$
E_{2}\left(\frac{a \tau+b}{c \tau+d}\right)=(c \tau+d)^{2} E_{2}(\tau)+\frac{6}{\pi \mathrm{i}} c(c \tau+d) .
$$

The modular property of the Gross-Taylor series was first observed in [48] by direct inspection of the Feynman diagram expansion for the free energy within the conformal field theory approach to large $N$ Yang-Mills theory 
proposed in [51]. A rigorous proof was subsequently presented in $[49,50]$ directly from the equivalent free fermion representation of the partition function [52]. Another proof, based on similar considerations, is presented in Appendix B. (see also [53]). The quasi-modular character of $F_{h}^{+}(\tau)$ and their computability through the Feynman diagram expansion in a KodairaSpencer field theory confirm two general predictions of the mirror symmetry program in the special case of elliptic curves [54]. In particular, the modular behaviour of the string expansion provides an interesting perspective on T-duality in the torus target space [51].

Explicit formulas for the free energy contributions up to genus $h=8$ are given in [48] (see also [47]). In a suitable basis for the modular forms, obtained from the identities

$$
\begin{aligned}
& E_{4}(\tau)=E_{2}(\tau)^{2}+12 E_{2}^{\prime}(\tau), \\
& E_{6}(\tau)=E_{2}(\tau)^{3}+18 E_{2}(\tau) E_{2}^{\prime}(\tau)+36 E_{2}^{\prime \prime}(\tau),
\end{aligned}
$$

they are given by the general expressions

$$
\begin{aligned}
& F_{1}^{+}(\lambda)=-\varepsilon_{\mathrm{F}}-\ln \eta(\tau), \\
& F_{h}^{+}(\lambda)=\frac{\lambda^{2 h-2}}{(2 h-2) ! \rho_{h}} \sum_{k=0}^{3 h-3} \sum_{\substack{l, m \in \mathbb{N}_{0} \\
2 l+3 m=3 h-3-k}} s_{h}^{k l} E_{2}(\tau)^{k} E_{2}^{\prime}(\tau)^{l} E_{2}^{\prime \prime}(\tau)^{m},
\end{aligned}
$$

with $h \geq 2$ and $\rho_{h}, s_{h}^{k l} \in \mathbb{N}$, where

$$
\varepsilon_{\mathrm{F}}=-\frac{\lambda}{24}\left(N^{2}-1\right)
$$

is the Fermi energy which in the Migdal expansion is the contribution from the vacuum Young diagram containing $i-1$ boxes in its $i$ th row and $\eta(\tau)$ the Dedekind function (3.5). By using the modular transformation properties

$$
\begin{aligned}
\eta\left(-\frac{1}{\tau}\right) & =\sqrt{-\mathrm{i} \tau} \eta(\tau), \\
E_{2}\left(-\frac{1}{\tau}\right) & =\tau^{2}\left(E_{2}(\tau)+\frac{6}{\pi \mathrm{i} \tau}\right), \\
E_{k}\left(-\frac{1}{\tau}\right) & =\tau^{k} E_{k}(\tau), \quad k=4,6, \ldots,
\end{aligned}
$$

it is possible to cast the string representation (5.8) and (5.9) in the weakcoupling regime $\lambda \ll 1$, where the instanton expansion of the gauge theory is appropriate. It was argued in [48] that the contributions are of the generic 
form

$$
F_{h}^{+}(\lambda)=\lambda^{2 h-2} \sum_{k=3 h-3}^{4 h-3} \frac{r_{k, h} \pi^{2(k-3 h+3)}}{\lambda^{k}}+\mathrm{O}\left(\mathrm{e}^{-1 / \lambda}\right)
$$

where $r_{k, h} \in \mathbb{Q}$ are related to the simple Hurwitz numbers. The precise geometrical meaning of the rational numbers $r_{k, h}$ will be elucidated in the next section.

Explicit expressions up to genus $h=6$, consistent with this structure, are found in [48]. Formula (5.21) represents the free energy contribution to the string expansion in the instanton picture, given as a coherent sum over magnetic charges (Chern classes) of $U(N)$ gauge bundles over $\mathbb{T}^{2}$. Rather surprisingly, the free energy displays a very mild singularity at $\lambda=0$, where the Douglas-Kazakov-type phase transition [34] would take place, indicating that the gauge string theory in the present case may possess some special hidden symmetries. In this limit, the gauge theory is related to a topological sigma-model with torus target space coupled to topological gravity [54], and it thereby becomes a topological string theory [55]. For generic values of the (dimensionless) Yang-Mills coupling constant $\lambda$, the string theory is described by a topological sigma-model perturbed by the Kähler class of the elliptic curve.

The rational numbers $r_{k, h}$ appearing in (5.21) will be computed in Section 5.3 using a saddle-point approximation of the zero-instanton sector of the weakly coupled gauge theory. An interesting problem is then the possibility of reconstructing the complete form of the free energy (5.18) directly in terms of the weak-coupling data, i.e., if it is possible, once the quasi-modular structure is assumed, to determine the natural numbers $\rho_{h}, s_{h}^{k l}$ from only the knowledge of the $r_{k, h}$. We will show in Section 5.4 that this is not possible and a certain number of parameters, determined in a definite way by a closed subspace of the vector space of modular forms, are therefore intrinsically related to non-trivial higher instanton contributions. This is somewhat surprising given the topological string character and the absence of a phase transition at finite area. The solution to this problem would yield the $\mathrm{QCD}_{2}$ analog of solutions to the recurrence relations encoded in the holomorphic anomaly equation for topological strings on Calabi-Yau manifolds [54].

\subsection{Instanton expansion of physical $U(N)$ gauge theory}

We will now work out the instanton expansion of the partition function for commutative $U(N)$ Yang-Mills theory on $\mathbb{T}^{2}[12,25,56]$, in a way that will be amenable for comparison later on with the string representation of the 
previous section. Starting from the general formula (2.12), we write the physical commutative partition function, summed over all topological Chern classes of $U(N)$ gauge bundles over the torus, as

$$
\begin{aligned}
Z_{U(N)}\left(g^{2} A\right):= & \mathrm{e}^{-\varepsilon_{\mathrm{F}}} \sum_{q=-\infty}^{\infty}(-1)^{N+(N-1) q} Z_{N, q}\left(g^{2} A, \theta=0\right) \\
= & (-1)^{N} \mathrm{e}^{-\varepsilon_{\mathrm{F}}} \sum_{\substack{\boldsymbol{\nu} \in \mathbb{N}_{0}^{N} \\
\sum_{k} k \nu_{k}=N}} \prod_{k=1}^{N} \frac{(-1)^{\nu_{k}}}{\nu_{k} !}\left(\frac{2 \pi^{2}}{k^{3} g^{2} A}\right)^{\nu_{k} / 2} \\
& \times \sum_{\boldsymbol{q} \in \mathbb{Z}^{|\boldsymbol{\nu}|}}(-1)^{(N-1) \sum_{k} q_{k}} \exp \left[-\frac{2 \pi^{2}}{g^{2} A} \sum_{l=1}^{N} \frac{1}{l} \sum_{j=1+\nu_{1}+\cdots+\nu_{l-1}}^{\nu_{1}+\cdots+\nu_{l}} q_{j}^{2}\right],
\end{aligned}
$$

where we have defined $\nu_{0}:=0$ and the total number of partition components $|\boldsymbol{\nu}|:=\nu_{1}+\cdots+\nu_{N}$. In (5.22), it is understood that if some $\nu_{k}=0$ then $q_{1+\nu_{1}+\cdots+\nu_{k-1}}=\cdots=q_{\nu_{1}+\cdots+\nu_{k}}=0$, while $\varepsilon_{\mathrm{F}}$ is the Fermi energy (5.19).

By defining the sequence of functions

$$
\Xi_{k}\left(g^{2} A\right):=\sum_{q=-\infty}^{\infty}(-1)^{(N-1) q} \mathrm{e}^{-\left(2 \pi^{2} / g^{2} A\right) q^{2} / k}
$$

for $k=1, \ldots, N$, we may bring the expansion (5.22) into the more compact form

$$
\begin{aligned}
Z_{U(N)}\left(g^{2} A\right)= & (-1)^{N} \mathrm{e}^{-\varepsilon_{\mathrm{F}}} \sum_{\substack{\boldsymbol{\nu} \in \mathbb{N}_{0}^{N} \\
\sum_{k} k \nu_{k}=N}} \prod_{k=1}^{N} \frac{(-1)^{\nu_{k}}}{\nu_{k} !}\left(\frac{2 \pi^{2}}{k^{3} g^{2} A}\right)^{\nu_{k} / 2} \\
& \times\left[\Xi_{k}\left(g^{2} A\right)\right]^{\nu_{k}} .
\end{aligned}
$$

Following the technique employed in Section 4.4, we may carry out the sum over partitions $\boldsymbol{\nu}$ in (5.24) explicitly by resolving the constraint using a contour integral representation (4.35), and thereby arrive at the formula

$$
Z_{U(N)}\left(g^{2} A\right)=\mathrm{e}^{-\varepsilon_{\mathrm{F}}} \oint_{\mathcal{C}_{0}} \frac{\mathrm{d} z}{2 \pi \mathrm{i} z^{N+1}} \exp \left[-\sqrt{\frac{2 \pi^{2}}{g^{2} A}} \sum_{k=1}^{\infty} \Xi_{k}\left(g^{2} A\right) \frac{(-z)^{k}}{k^{3 / 2}}\right] .
$$

This representation of the weak-coupling partition function, valid for all values of $N$ thanks to the contour integration, will be the key to extract its form in the desired large $N$ scaling limits. 
For later comparison with the non-commutative setting, let us derive the analog of the contour integral formula (5.25) for the strong-coupling expansion of the commutative $U(N)$ gauge theory. For this, we use the Poisson resummation formula

$$
\sum_{q=-\infty}^{\infty} f(q)=\sum_{n=-\infty}^{\infty} \int_{-\infty}^{\infty} \mathrm{d} s f(s) \mathrm{e}^{2 \pi \mathrm{i} n s}
$$

to rewrite the sequence of functions $(5.23)$ as

$$
\Xi_{k}\left(g^{2} A\right)=\sqrt{\frac{k g^{2} A}{2 \pi}} \sum_{n=-\infty}^{\infty} \mathrm{e}^{-\left(g^{2} A / 2\right)\left(n-n_{\mathrm{F}}\right)^{2} k},
$$

where we have defined the Fermi surface level

$$
n_{\mathrm{F}}=\frac{N-1}{2}
$$

This simple transformation rule is of course a consequence of the fact that the functions (5.23) define modular Jacobi theta-functions on the elliptic curve of the previous section. By substituting (5.27) into (5.25), the sum over $k$ can be performed explicitly and one finally arrives at the strong-coupling form

$$
Z_{U(N)}\left(g^{2} A\right)=\mathrm{e}^{-\varepsilon_{\mathrm{F}}} \oint_{\mathcal{C}_{0}} \frac{\mathrm{d} z}{2 \pi \mathrm{i} z^{N+1}} \prod_{n=-\infty}^{\infty}\left(1+z \mathrm{e}^{-\left(g^{2} A / 2\right)\left(n-n_{\mathrm{F}}\right)^{2}}\right) .
$$

Expression (5.29) represents a concise resummation of the Migdal expansion of commutative $U(N)$ gauge theory on $\mathbb{T}^{2}$, with the contour integration implementing the constraint that the number of rows in the Young diagrams be bounded from above by the rank $N$ of the gauge group. This may be checked explicitly by expanding the product in (5.29) and evaluating the integral. This formula agrees with the representation derived in [57] by direct group theory arguments and it is also similar to the contour integral formula obtained in [51] from the free fermion representation of the partition function. The free fermion formula can also be derived directly from the combinatorics of branched covers [49] and from a complex matrix model which serves as a generating function for Hurwitz numbers [58].

\subsection{Saddle point solution}

We will now compute the partition function (5.25) in the large $N$ limit with the 't Hooft coupling constant (5.2) held fixed and compare it to the string representation of Section 5.1. In the weak-coupling limit, the higher instanton contributions to the function (5.23) are of order $\mathrm{e}^{-N / \lambda}$ and hence 
can be neglected to a first-order approximation at $N \rightarrow \infty$. We may thereby focus on the zero-instanton sector with vanishing magnetic charge $q=0$ and write the vacuum amplitude in terms of the polylogarithm function (4.38) as

$$
\mathcal{Z}_{U(N)}(\lambda)=\mathrm{e}^{-\varepsilon_{\mathrm{F}}} \oint_{\mathcal{C}_{0}} \frac{\mathrm{d} z}{2 \pi \mathrm{i} z} \exp \left[-N \ln z-\sqrt{\frac{\pi N}{\lambda}} \operatorname{Li}_{3 / 2}(-z)\right] .
$$

Note that, in contrast to the case of Yang-Mills theory on the sphere [35, 36], the zero-instanton contribution on $\mathbb{T}^{2}$ is an infinite series when $N$ is large (through the expansion of $\mathrm{Li}_{3 / 2}(-z)$ ) owing to the topological degeneracy of the instanton configurations in this case. We will compute the integral (5.30) in the large $N$ limit by means of saddle-point techniques. The vanishing of the first derivative with respect to $z$ of the exponential function gives the saddle-point equation

$$
\operatorname{Li}_{1 / 2}(-z)=-\sqrt{\frac{N \lambda}{\pi}}
$$

By using the integral representation of Appendix A, i.e., equation (A.2), one can show that the solutions $z=z_{*}$ of this equation are necessarily real for all values of the coupling $\lambda$. In fact, the function $\operatorname{Li}_{1 / 2}(-z)$ is a slowly decreasing negative function for $z \geq 1$, behaving as [59] $\mathrm{Li}_{1 / 2}(-z) \simeq-2 \sqrt{\ln (z) / \pi}$ for $z$ real with $z \rightarrow \infty$ (figure 3 ). Thus a solution to (5.31) always exists and is located at large $z \in[-1, \infty)$.

The saddle-point equation (5.31) can be solved to any order in $N$ by using the large $x$ asymptotic expansion of the polylogarithm function

$$
\operatorname{Li}_{\alpha}\left(-\mathrm{e}^{x}\right)=2 \sum_{k=0}^{\infty} \frac{\left(1-2^{2 k-1}\right) B_{2 k} \pi^{2 k}}{(2 k) ! \Gamma(\alpha+1-2 k)} x^{\alpha-2 k}
$$

which we derive in Appendix A. Corrections to this formula are of order $\mathrm{e}^{-x}$. We seek a solution of the form $z=z_{*}=\mathrm{e}^{x_{*}}$ with $x_{*}$ admitting an asymptotic $1 / N$ expansion

$$
x_{*}=x_{-1} N+\sum_{k=0}^{\infty} \frac{x_{k}}{N^{k}} .
$$

The series (5.33) starts at order $N$ due to the form of the leading term in the asymptotic expansion (5.32). One can now proceed to obtain the coefficients $x_{k}$ of the saddle-point solution recursively in powers of $1 / N$ by substituting (5.32) and (5.33) into (5.31). The result of a straightforward 


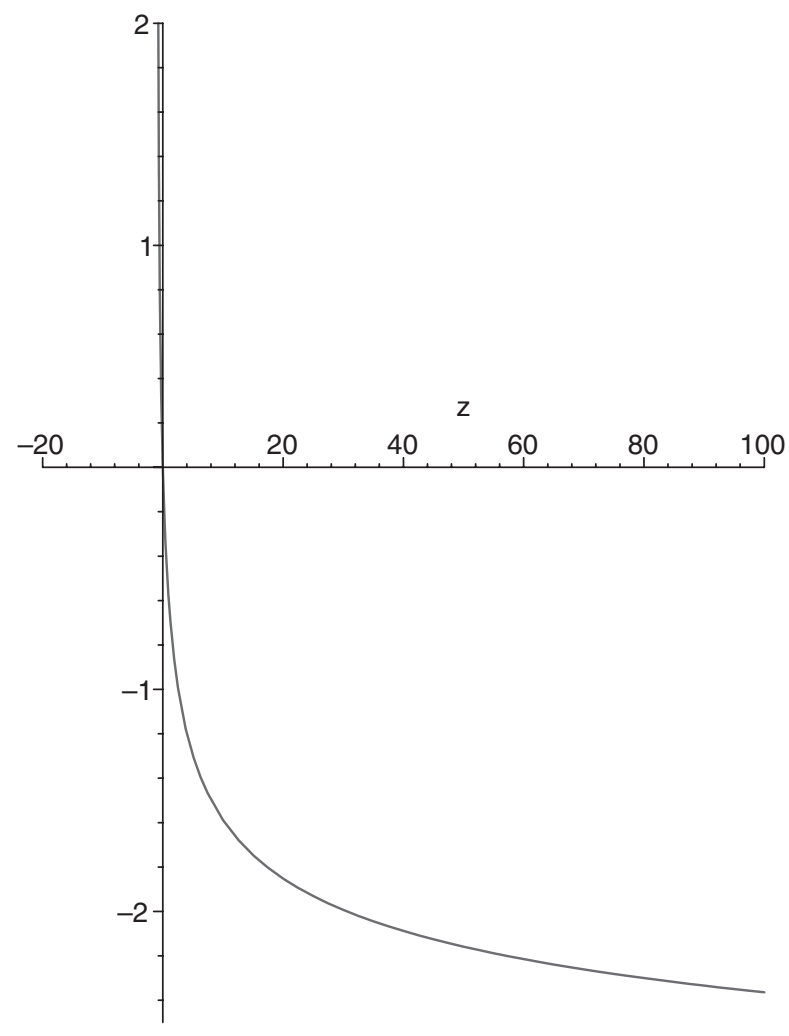

Figure 3: The Jonquière function $\operatorname{Li}_{1 / 2}(-z)$ versus $z \in(-1, \infty)$.

iterative evaluation up to order $1 / N^{11}$ reads

$$
\begin{aligned}
x_{*}= & \frac{N \lambda}{4}+\frac{\pi^{2}}{3 N \lambda}+\frac{16 \pi^{4}}{9(N \lambda)^{3}}+\frac{448 \pi^{6}}{9(N \lambda)^{5}}+\frac{1254656 \pi^{8}}{405(N \lambda)^{7}} \\
& +\frac{406598656 \pi^{10}}{1215(N \lambda)^{9}}+\frac{67556569088 \pi^{12}}{1215(N \lambda)^{11}}+\mathrm{O}\left(\frac{1}{N^{13}}\right) .
\end{aligned}
$$

The systematic vanishing of the $x_{k}$ for even powers of $1 / N$ is exactly what is expected from the form of the Gross-Taylor expansion.

Armed with the solution (5.34) of the saddle-point equation, we can now proceed to compute the free energy $\mathcal{F}_{U(N)}(\lambda)=\ln \mathcal{Z}_{U(N)}(\lambda)$ by parametrizing the integration variable in (5.30) as $z=\mathrm{e}^{x_{*}+x}$, where the variable $x$ contains contributions from fluctuations about the saddle-point value. The saddle-point itself is at $x=0$ and we can integrate the fluctuations over all $x \in \mathbb{R}$, since the deviations from the results computed with the correct integration domain appropriate to (5.30) will be exponentially suppressed 
in the small area limit. By using (5.32), the computation of

$$
\mathcal{F}_{U(N)}(\lambda)=\ln \left(\mathrm{e}^{-\varepsilon_{\mathrm{F}}} \int_{-\infty}^{\infty} \mathrm{d} x \exp \left[-N\left(x_{*}+x\right)-\sqrt{\frac{\pi N}{\lambda}} \mathrm{Li}_{3 / 2}\left(-\mathrm{e}^{x_{*}+x}\right)\right]\right)
$$

as an expansion up to order $1 / N^{10}$ is straightforward to do, but quite cumbersome. A numerical evaluation using Mathematica yields

$$
\begin{aligned}
\mathcal{F}_{U(N)}(\lambda)= & \frac{1}{2} \ln (-2 \pi \lambda)+\frac{\pi^{2}}{6 \lambda}+\left(\frac{2}{3 \lambda}-\frac{2 \pi^{2}}{3 \lambda^{2}}+\frac{8 \pi^{4}}{45 \lambda^{3}}\right) \frac{1}{N^{2}} \\
& +\left(-\frac{8}{\lambda^{2}}+\frac{16 \pi^{2}}{\lambda^{3}}-\frac{100 \pi^{4}}{9 \lambda^{4}}+\frac{224 \pi^{6}}{81 \lambda^{5}}\right) \frac{1}{N^{4}} \\
& +\left(\frac{2272}{9 \lambda^{3}}-\frac{2272 \pi^{2}}{3 \lambda^{4}}+\frac{8096 \pi^{4}}{9 \lambda^{5}}-\frac{41504 \pi^{6}}{81 \lambda^{6}}+\frac{48256 \pi^{8}}{405 \lambda^{7}}\right) \frac{1}{N^{6}} \\
& +\left(-\frac{13504}{\lambda^{4}}+\frac{54016 \pi^{2}}{\lambda^{5}}-\frac{834304 \pi^{4}}{9 \lambda^{6}}\right. \\
& \left.+\frac{7010816 \pi^{6}}{81 \lambda^{7}}-\frac{17887904 \pi^{8}}{405 \lambda^{8}}+\frac{11958784 \pi^{10}}{1215 \lambda^{9}}\right) \frac{1}{N^{8}} \\
+ & \left(\frac{15465472}{15 \lambda^{5}}-\frac{15465472 \pi^{2}}{3 \lambda^{6}}+\frac{105156608 \pi^{4}}{9 \lambda^{7}}-\frac{418657280 \pi^{6}}{27 \lambda^{8}}\right. \\
& \left.+\frac{572409344 \pi^{8}}{45 \lambda^{9}}-\frac{2467804672 \pi^{10}}{405 \lambda^{10}}+\frac{33778284544 \pi^{12}}{25515 \lambda^{11}}\right) \frac{1}{N^{10}} \\
+ & \mathrm{O}\left(\frac{1}{N^{12}}\right) \cdot
\end{aligned}
$$

Expression (5.36) matches precisely (3.43)-(3.47) in [48] which were obtained using the conformal field theory representation of large $N$ twodimensional Yang-Mills theory. The saddle-point evaluation of the zeroinstanton sector thereby reproduces all non-exponentially suppressed terms in the weak-coupling expansion of the chiral $U(N)$ free energy, giving the correct rational numbers $r_{k, h}$ appearing in (5.21). An important ingredient in this reproduction is the cancellation of the ground-state energy (5.19) that appears in (5.35), since this term would otherwise dominate the series in the large $N$ limit. In particular, the expansion starts at order $N^{0}$ and there is no spherical contribution. Expansion (5.36) also contains the correct leading modular dependence of the Dedekind function coming from the genus 1 free energy. 
The derivation of formula (5.36) that we have presented here has three particularly noteworthy features. First, it is a highly accurate check of the expansion obtained in [48] by a completely independent method. Second, it does not rely on any group theory or conformal field theory techniques and is obtained directly in the instanton representation. As far as we are aware, this is the first time that stringy quantities are computed in twodimensional Yang-Mills theory directly from the weak-coupling expansion, which is the most natural one from a conventional quantum field theory point of view. This is possible, of course, due to the absence of a phase transition at finite area on the torus, as on the sphere the Douglas-Kazakov transition prohibits the recovery of stringy features from the weak-coupling data [34-36]. Finally, we note that the dynamics of the zero-instanton sector is surprisingly rich, encoding properly the anticipated stringy features. This is most likely related to the underlying structure of the topological string theory governing the weak-coupling limit [55].

However, despite this remarkable agreement, there is a serious flaw in the computation above. Evidently, we have reproduced only the chiral part of the full $U(N)$ gauge theory. The saddle-point technique has not picked up the coupling to the anti-chiral sector. Furthermore, after some calculation one can find that even in the exponentially suppressed contributions, there are terms missing in the saddle-point analysis which prevent a resummation to the full quasi-modular Dedekind function and Eisenstein series. Part of the problem can be traced back to the fact that the argument of the exponential integrand in (5.30) does not admit a nice large $N$ scaling of the form $\mathrm{e}^{-N f(z ; \lambda)}$, with the function $f(z ; \lambda)$ independent of $N$. In evaluating the fluctuation integral (5.35), there are Gaussian terms of the form $\mathrm{e}^{-x^{2} / \lambda}$ which thus arise and are independent of $N$. In order for the saddle-point technique to be reliable, the widths of such Gaussians should vanish as $N \rightarrow \infty$ in order for the solution to localize around the saddle-point value at $x=0$. The difficulty stems from the linear growth in $N$ of the position of the saddle-point (5.33).

Moreover, we eventually have to face up to the problem of evaluating the non-zero higher instanton contributions. It is very likely that, in spite of their exponential suppression order by order in $1 / N$, their collective behaviour will be crucial to recover the complete string expansion. Recall that instantons are essential to the recovery of the string picture of large $N$ Yang-Mills theory formulated on the sphere [36]. We have not accomplished an analytical evaluation of the full instanton series at large $N$. However, we can address the problem of how much information should be carried by the higher instantons in order to recover the full string partition function. This is the topic of the following section. 


\subsection{Quasi-modularity of higher instanton configurations}

On spacetimes of genus $\geq 2$, the partition function of Yang-Mills theory in two dimensions is smooth in the limit of vanishing area and it defines a topological string theory [55]. This process can also be reversed and the partition function (and observables) at finite area can be expressed in terms of correlators computed in this topological string theory which are related to intersection numbers in the Hurwitz moduli spaces of topological classes of branched covers. Instead, it is not obvious that this procedure is reliable on the torus, since the weak-coupling limit of the partition function is singular in that case. However, one may ask what amount of information is encoded in the singular terms and if it is enough to reconstruct the whole partition function. In what follows, we shall answer this question using only the knowledge that $F_{h}^{+}(\tau)$ is a quasi-modular form of weight $6 h-6$.

For this, let us first recall some basic facts about quasi-modular forms [32]. Let $k \in 2 \mathbb{N}_{0}$ and $s \in \mathbb{N}_{0}$. A quasi-modular form of weight $k$ and level $s$ is a holomorphic function $f: \mathbb{C}_{+} \rightarrow \mathbb{C}$ on the upper complex half-plane such that

$$
(c \tau+d)^{-k} f\left(\frac{a \tau+b}{c \tau+d}\right)=\sum_{n=0}^{s} f_{n}(\tau)\left(\frac{c}{c \tau+d}\right)^{n},
$$

where the functions $f_{n}(\tau), 1 \leq n \leq s$, are independent of the modular transformation matrices (5.15) and $f_{0}(\tau):=f(\tau)$. A quasi-modular form of level 0 is a modular form. We denote the complex vector space of quasi-modular forms of weight $k$ and level $\leq s$ by $\mathcal{M}_{k}^{s}$. The product of a quasi-modular form in $\mathcal{M}_{k_{1}}^{s_{1}}$ with a quasi-modular form in $\mathcal{M}_{k_{2}}^{s_{2}}$ is a quasi-modular form in $\mathcal{M}_{k_{1}+k_{2}}^{s_{1}+s_{2}}$. Then the vector space

$$
\mathcal{M}:=\bigoplus_{k \in 2 \mathbb{N}_{0}} \bigoplus_{s=0}^{\infty} \mathcal{M}_{k}^{s}
$$

naturally becomes a graded algebra. The general structure of this algebra is provided by the following central result.

Theorem 1. Every quasi-modular form $f(\tau)$ of weight $k$ and level $s$ can be written as

$$
f(\tau)=\sum_{l=0}^{s} E_{2}(\tau)^{l} M_{k-2 l}(\tau),
$$

where $M_{k-2 l}(\tau)$ is a modular form of weight $k-2 l$.

Theorem 1 implies that the ring $\mathcal{M}$ of quasi-modular forms is the graded algebra generated over the $\operatorname{ring} \mathcal{M}^{0}:=\bigoplus_{k \in 2 \mathbb{N}_{0}} \mathcal{M}_{k}^{0}$ of modular forms by the 
Eisenstein series of weight 2 as

$$
\mathcal{M}=\mathcal{M}^{0} \otimes \mathbb{C}\left[E_{2}\right]
$$

The modular anomaly of the Eisenstein series (5.16) leads to the requirement that the genus $h$ free energy $F_{h}^{+}(\tau)$ be a quasi-modular form of weight $k=6 h-6$ and level $s=3 h-3$. Theorem 1 then constrains the effects of the gauge dynamics in a finite set of parameters, implying that the free energy can be written as

$$
F_{h}^{+}(\tau)=\sum_{l=0}^{3 h-3} E_{2}(\tau)^{l} M_{6 h-6-2 l}(\tau),
$$

where $M_{6 h-6-2 l}(\tau)$ is a (true) modular form of weight $6 h-6-2 l$ (or a weight $6 h-6-2 l$ combination of $E_{2}^{\prime}(\tau)$ and $E_{2}^{\prime \prime}(\tau)$ in the alternative basis of Section 5.1). The finite dimension of the $\mathbb{C}$-linear space $\mathcal{M}_{k}^{0}$ of modular forms of weight $k$ is given by [32]

$$
\operatorname{dim} \mathcal{M}_{k}^{0}=1-\frac{k}{2}+\left\lfloor\frac{k}{3}\right\rfloor+\left\lfloor\frac{k}{4}\right\rfloor .
$$

The number of free parameters in the expansion (5.40) can then be easily evaluated by computing the sum

$$
K_{h}^{\#}:=\sum_{l=0}^{3 h-3} \operatorname{dim} \mathcal{M}_{6 h-6-2 l}^{0}= \begin{cases}\frac{1}{4}\left(3 h^{2}+1\right) & \text { for } h \text { odd } \\ \frac{3}{4} h^{2} & \text { for } h \text { even }\end{cases}
$$

We are interested in this structure in the weak-coupling limit $\lambda \rightarrow 0$. As in Section 5.1, it is straightforward to extract the small area behaviour of the free energy at genus $h$ from the representation (5.40) in terms of quasimodular forms, and for generic coefficients one finds the general expansion

$$
F_{h}^{+}(\lambda)=\sum_{l=3 h-3}^{6 h-6} \frac{m_{l, h}}{\lambda^{l}}+\mathrm{O}\left(\mathrm{e}^{-1 / \lambda}\right),
$$

where $m_{l, h} \in \mathbb{R}$ are linear combinations of the $K_{h}^{\#}$ free parameters in (5.40). This is to be compared with the instanton representation (5.21), which singles out the singular behaviour of the free energy at weak coupling as a polynomial in $1 / \lambda$ of degree $4 h-3$ with leading term of order $3 h-3$. Thus matching the two expansions will provide (at most) $3 h-2$ conditions on the coefficients appearing in the expansion (5.40).

To determine the constraints arising from the small area behaviour, it is convenient to choose an adapted basis for the space of modular forms. For 
this, we decompose $\mathcal{M}_{k}^{0}$ as a vector space in the form [32]

$$
\mathcal{M}_{k}^{0}=\mathbb{C} \cdot E_{k} \oplus \mathcal{M}_{k-12}^{0} \cdot \triangle
$$

where the Ramanujan modular form

$$
\triangle(\tau):=E_{4}(\tau)^{3}-E_{6}(\tau)^{2}=1728 \eta(\tau)^{24}
$$

of weight 12 is the discriminant of the underlying elliptic curve. Equation (5.44) implies that every modular form of weight $k$ can be written as a complex linear combination of the weight $k$ Eisenstein series (5.13) with a modular form which is the product of (5.45) and a modular form of weight $k-12$. Representation (5.40) can be rewritten using this decomposition as

$$
F_{h}^{+}(\tau)=\sum_{l=0}^{3 h-4} c_{l, h} E_{2}(\tau)^{l} E_{6 h-6-2 l}(\tau)+\triangle(\tau) \sum_{l=0}^{3 h-3} E_{2}(\tau)^{l} M_{6 h-18-2 l}(\tau) .
$$

The remarkable property of this expansion for the genus $h$ free energy is that the small area behaviour is completely dictated by the first contribution in (5.46), as the discriminant $\triangle(\tau)$ is exponentially suppressed at both weak and strong coupling. Therefore, matching (5.43) with (5.21) fixes the $3 h-3$ real coefficients $c_{l, h}$ in (5.46). Note that this procedure is not guaranteed to work, as there are $3 h-3$ unknowns but only $3 h-2$ conditions. Some sort of miraculous reduction should (and will) occur.

The weak-coupling limit of (5.46) can thereby be straightforwardly worked out using the modular transformation properties (5.37), analogous to Section 5.1, and after some algebra one finds

$$
\begin{aligned}
F_{h}^{+}(\lambda)= & \sum_{l=0}^{3 h-5} \frac{\left(-4 \pi^{2}\right)^{l+2}(12)^{3 h-5-l}}{\lambda^{3 h-1+l}}\left[\left(\begin{array}{c}
3 h-3 \\
l+2
\end{array}\right) c_{3 h-4, h}\right. \\
& \left.+\sum_{l^{\prime}=0}^{l}\left(\begin{array}{c}
3 h-5-l^{\prime} \\
l-l^{\prime}
\end{array}\right) c_{3 h-5-l^{\prime}, h}\right]+\frac{(12)^{3 h-3} c_{3 h-4, h}}{\lambda^{3 h-3}} \\
& -\frac{4 \pi^{2}(3 h-3)(12)^{3 h-4} c_{3 h-4, h}}{\lambda^{3 h-2}}+\mathrm{O}\left(\mathrm{e}^{-1 / \lambda}\right)
\end{aligned}
$$

From this expression, it is clear that expansion (5.46) can match (5.43) if and only if the ratio of the two coefficients of the lowest singularity in (5.47) and (5.43) is fixed and equal to $-\pi^{2}(h-1)$. One can check explicitly using (5.40) that this is indeed the case (this is the miraculous reduction in the number of conditions that we mentioned above). The fact that this criterion is satisfied is also a check that the singularities we found through the saddle-point computation of the previous section originate from a quasi-modular form. 
At this point, starting from the lowest singularity in (5.47), we can compare with (5.43) and recursively determine all coefficients of the small area expansion. Then the number of free parameters that remain undetermined is given by

$$
\Delta K_{h}^{\#}=K_{h}^{\#}-(3 h-3)= \begin{cases}\frac{1}{4}\left(3(h-2)^{2}+1\right) & \text { for } h \text { odd } \\ \frac{3}{4}(h-2)^{2} & \text { for } h \text { even. }\end{cases}
$$

For example, $\Delta K_{2}^{\#}=0$ and thus the genus 2 free energy is completely determined by its behaviour at weak coupling. At genus 3 there is $\Delta K_{3}^{\#}=1$ free parameter, at genus 4 there are $\Delta K_{4}^{\#}=3$ parameters, and so on. We are therefore led to conclude that higher instantons should account for the remaining undetermined dynamical parameters in the weak-coupling expansion of the gauge theory. Furthermore, the exponentially suppressed contributions, which are not related to the small area behaviour and are required to reinstate the full quasi-modular structure at genus $h$, are parametrized by higher instanton contributions living in the subspace

$$
\mathcal{P}_{h}:=\bigoplus_{l=0}^{3 h-3} \mathcal{M}_{6 h-18-2 l}^{0} \cdot\left(E_{2}\right)^{l} \triangle
$$

of the graded algebra (5.38).

\section{The double-scaling limit}

Motivated by the analysis of the previous section, we will now analyse the $U(N)$ gauge theory in a large $N$ limit wherein the saddle-point technique can capture the entire relevant story. It is clear what to do. We should take the limit $N \rightarrow \infty$ while keeping fixed the new scaled coupling constant

$$
\mu:=\frac{N \lambda}{\pi}=\frac{N^{2} g^{2} A}{2 \pi} .
$$

The partition function then assumes the form

$$
\begin{aligned}
\widehat{\mathcal{Z}}_{U(N)}(\mu) & =\mathrm{e}^{\pi N \mu / 12} \oint_{\mathcal{C}_{0}} \frac{\mathrm{d} z}{2 \pi \mathrm{i} z} \exp \left[-N\left(\ln z+\frac{1}{\sqrt{\mu}} \operatorname{Li}_{3 / 2}(-z)\right)\right] \\
& =: \oint_{\mathcal{C}_{0}} \frac{\mathrm{d} z}{2 \pi \mathrm{i} z} \mathrm{e}^{N \widehat{F}(z, \mu)},
\end{aligned}
$$

and hence has a nice large $N$ limit. Corrections to this expression from higher instanton configurations are of order $\exp \left(-N^{2} / \mu\right)$ and could be completely suppressed in the $1 / N$ expansion. In fact, at $N=\infty$ the vacuum amplitude is given by the leading planar term $\widehat{\mathcal{Z}}_{U(N)}(\mu)=\mathrm{e}^{N \widehat{F}\left(z_{*}, \mu\right)}$ in the 
$1 / N$ expansion of the integral (6.2), which can be rigorously computed in the saddle-point approximation. We will refer to this new limit of the gauge theory as the "double-scaling limit", because it is the limit appropriate for the mapping onto non-commutative Yang-Mills theory which will be carried out in the next section.

This limit is very different from the conventional planar large $N$ limit used to derive the Gross-Taylor expansion. In this section, we will analyse this double scaling in the simpler $U(N)$ theory in order to explore the fate of the string expansion in this new limit of the gauge theory. Again we shall see that there is a non-trivial saddle point governing the large $N$ behaviour of the zero-instanton sector. We will indeed find that an open string representation potentially emerges with expansion coefficients completely determined, in the limit of infinite winding number, by the geometrical quantities (Hurwitz numbers) parametrizing the closed strings on the torus. The open string theory is deeply tied to the geometry of a particular class of moduli spaces of Riemann surfaces which we describe in detail. This will be the crux of the string expansion of non-commutative gauge theory that we will describe in the next section.

\subsection{Saddle point solution}

Starting from (6.2), we derive the saddle-point equation

$$
\operatorname{Li}_{1 / 2}(-z)=-\sqrt{\mu}
$$

and it can be solved exactly, at least in principle. In contrast to the conventional 't Hooft limit, this equation does not depend on $N$ and therefore its solution $z=z_{*}(\mu)$ does not rely on any approximation in general. It can be simply written as $z_{*}(\mu)=-\mathrm{Li}_{1 / 2}^{-1}(-\sqrt{\mu})$, the inverse function being uniquely defined in the region of interest thanks to the monotonic behaviour of the polylogarithm function $\mathrm{Li}_{1 / 2}(-z)$. The double-scaling free energy can then be evaluated as $\widehat{\mathcal{F}}_{U(N)}(\mu)=N \widehat{F}\left(z_{*}(\mu), \mu\right)$ and is depicted in figure 4. It is a smooth function of $\mu>0$, with a logarithmic singularity in the weak-coupling limit $\mu \rightarrow 0$, where the Douglas-Kazakov-type phase transition takes place. In fact, using properties of the Jonquière function (see Appendix A.), we can write down an exact relation that gives the free energy directly as a primitive of the position of the saddle point. By parametrizing the saddle-point solution as before in the form $z_{*}(\mu)=\mathrm{e}^{x_{*}(\mu)}$ and defining $y:=\sqrt{\mu}$, one can easily derive the equation

$$
\widehat{F}\left(z_{*}(\mu), \mu\right)=\frac{1}{\sqrt{\mu}} \int_{\sqrt{\mu}}^{\infty} \mathrm{d} y\left[x_{*}\left(y^{2}\right)-\frac{\pi y^{2}}{4}\right] .
$$




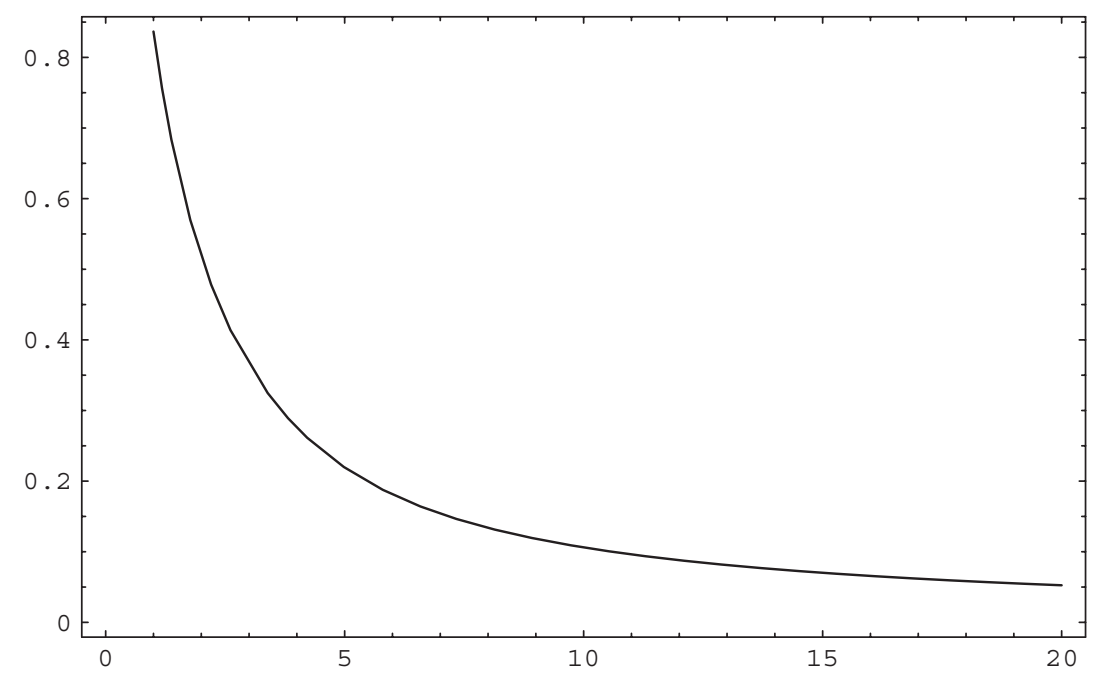

Figure 4: The double-scaling free energy function $\widehat{F}\left(z_{*}(\mu), \mu\right)$ versus $\mu$.

This explicit representation is useful because it exhibits the structure of the free energy straightforwardly in terms of the properties of the saddle-point solution.

The physical meaning of the double-scaling limit can be understood by expanding the free energy for large $\mu$. It is in this regime that we expect to see a relation with the string picture derived previously in the 't Hooft scaling limit. The strong-coupling saddle-point solution is given by the expansion (5.34) which is naturally written as a series in the double-scaling parameter given by

$$
x_{*}(\mu)=\pi \sum_{k=0}^{\infty} \frac{\xi_{2 k-1}}{\mu^{2 k-1}}
$$

with $\xi_{2 k-1} \in \mathbb{Q}$. The double-scaling free energy $\widehat{\mathcal{F}}_{U(N)}$ may then be computed directly from (6.4) to get

$$
\widehat{\mathcal{F}}_{U(N)}(\mu)=\pi N\left[\sum_{k=1}^{\infty} \frac{\xi_{2 k-1}}{4 k-3} \frac{1}{\mu^{2 k-1}}+\mathrm{O}\left(\mathrm{e}^{-\mu}\right)\right],
$$

where we note the cancellation of the vacuum energy contribution. From the explicit expression in (5.34), the first few terms are found to be given by

$$
\begin{aligned}
\widehat{\mathcal{F}}_{U(N)}(\mu)= & 2 \pi N\left[\frac{1}{6 \mu}+\frac{8}{45 \mu^{3}}+\frac{224}{81 \mu^{5}}+\frac{48256}{405 \mu^{7}}\right. \\
& \left.+\frac{11958784}{1215 \mu^{9}}+\frac{33778284544}{25515 \mu^{11}}+\mathrm{O}\left(\frac{1}{\mu^{13}}\right)+\mathrm{O}\left(\mathrm{e}^{-\mu}\right)\right] .
\end{aligned}
$$


By momentarily disregarding the exponentionally suppressed contributions to (6.7) and comparing with (5.36), we see that at strong-coupling the double scaling limit has extracted the most singular terms, as $\lambda \rightarrow 0$, at each order of the original $1 / N$ expansion. In other words, the double-scaled gauge theory at strong coupling presents a resummation of the most singular terms in the weak-coupling limit of the chiral Gross-Taylor string expansion. By substituting (6.1) into (5.21), the leading contribution there at $N=\infty$ is given at $k=4 h-3$, and thus the general form of this expansion can be written as

$$
\widehat{\mathcal{F}}_{U(N)}(\mu)=\pi N\left[\sum_{h=1}^{\infty} \frac{r_{4 h-3, h}}{\mu^{2 h-1}}+\mathrm{O}\left(\mathrm{e}^{-\mu}\right)\right] .
$$

From (6.6), it then follows that the rational numbers $r_{4 h-3, h}$ are completely determined by the strong-coupling solution (6.5) of the saddle-point equation to be

$$
r_{4 h-3, h}=\frac{\xi_{2 h-1}}{4 h-3}
$$

We will now compute the explicit forms of the coefficients of the saddlepoint expansion (6.5) and (6.6) as polynomials in Bernoulli numbers, and thereby write down the exact solution of the double-scaling gauge theory in the strong-coupling limit. For this, we set $x_{*}=1 / w^{2}$ and use (5.32) to write the saddle-point equation (6.3) as

$$
w=-\frac{2}{\sqrt{\mu}} \sum_{k=0}^{\infty} \frac{\left(1-2^{2 k-1}\right) B_{2 k} \pi^{2 k}}{(2 k) ! \Gamma\left(\frac{3}{2}-2 k\right)} w^{4 k}=: \frac{1}{\sqrt{\mu}} L(w) .
$$

The solution of the saddle-point equation to all orders in the strong-coupling regime is thereby reduced to the formal inversion of a Taylor series. This problem may be solved by standard Lagrange inversion [60], which gives the formal solution of (6.10) as the infinite series

$$
w(\mu)=\left.\sum_{k=0}^{\infty} \frac{1}{(k+1) !} \frac{\mathrm{d}^{k}}{\mathrm{~d} z^{k}} L(z)^{k+1}\right|_{z=0}\left(\frac{1}{\sqrt{\mu}}\right)^{k+1} .
$$

The solution (6.11) can be rewritten in a more compact form by means of the contour integral

$$
w(\mu)=\oint_{\mathcal{C}_{0}} \frac{\mathrm{d} z}{2 \pi \mathrm{i}} z \frac{1-(1 / \sqrt{\mu}) L^{\prime}(z)}{z-(1 / \sqrt{\mu}) L(z)},
$$

where now the contour $\mathcal{C}_{0}$ encircles both the origin $z=0$ and the zero of the denominator in (6.12). The equivalence of the two expressions (6.11) and (6.12) is straightforwardly established by writing a formal Taylor series expansion of the integrand in (6.12) in powers of $1 / \sqrt{\mu}$ and computing the integral. 
For our purposes, it will be more useful to employ the Bürmann generalization of the Lagrange inversion formula [60]. The contour integral representation (6.12) can be easily generalized to evaluate any analytic function $G$ of the solution $w(\mu)$ by writing

$$
G(w(\mu))=\oint_{\mathcal{C}_{0}} \frac{\mathrm{d} z}{2 \pi \mathrm{i}} G(z) \frac{1-(1 / \sqrt{\mu}) L^{\prime}(z)}{z-(1 / \sqrt{\mu}) L(z)} .
$$

To compute $x_{*}(\mu)=1 / w(\mu)^{2}$, we should take $G(z)=1 / z^{2}$, but this cannot be directly inserted into the formula (6.13) as it would introduce a spurious contribution from the double pole at $z=0$. The simplest way to deal with this problem is to subtract the undesired contribution by hand, and thereby write the solution $x_{*}(\mu)$ of the saddle-point equation as

$$
x_{*}(\mu)=\oint_{\mathcal{C}_{0}} \frac{\mathrm{d} z}{2 \pi \mathrm{i} z^{2}} \frac{1-(1 / \sqrt{\mu}) L^{\prime}(z)}{z-(1 / \sqrt{\mu}) L(z)}-\frac{L(0) L^{\prime \prime}(0)-\left(\sqrt{\mu}-L^{\prime}(0)\right)^{2}}{L(0)^{2}} .
$$

By formally expanding (6.14) in powers of $1 / \sqrt{\mu}$ and using the definition of the function $L(z)$ in (6.10), we arrive at the strong-coupling solution

$$
\begin{aligned}
x_{*}(\mu) & =-\frac{L(0) L^{\prime \prime}(0)-\left(\sqrt{\mu}-L^{\prime}(0)\right)^{2}}{L(0)^{2}}-\left.2 \sum_{k=1}^{\infty} \frac{1}{(k+2) ! k} \frac{\mathrm{d}^{k+2}}{\mathrm{~d} z^{k+2}} L(z)^{k}\right|_{z=0} \frac{1}{\mu^{k / 2}} \\
& =\frac{\pi \mu}{4}-2 \sum_{k=1}^{\infty} \frac{L_{k+2}^{(k)}}{k} \frac{1}{\mu^{k / 2}},
\end{aligned}
$$

where the coefficients

$$
L_{k+2}^{(k)}=\sum_{\substack{k \in \mathbb{N}_{0}^{k} \\ 4 \sum_{i} k_{i}=k+2}} \prod_{i=1}^{k} \frac{\left(2-2^{2 k_{i}}\right) B_{2 k_{i}} \pi^{2 k_{i}}}{\left(2 k_{i}\right) ! \Gamma\left(\frac{3}{2}-2 k_{i}\right)}
$$

are non-zero only when $k=4 h-2$ for some $h \in \mathbb{N}$.

Written in the form of (6.5), one has $\xi_{-1}=\frac{1}{4}$ as anticipated, while

$$
\xi_{2 h-1}=-\frac{L_{4 h}^{(4 h-2)}}{\pi(2 h-1)}=-\frac{\pi^{2 h-1}}{2 h-1} \sum_{\substack{h \in \mathbb{N}_{0}^{4 h-2} \\ \sum_{k} h_{k}=h}} \prod_{k=1}^{4 h-2} \frac{\left(2-2^{2 h_{k}}\right) B_{2 h_{k}}}{\left(2 h_{k}\right) ! \Gamma\left(\frac{3}{2}-2 h_{k}\right)}
$$

for $h \geq 1$, and we have thereby found the complete strong-coupling expansion of the double scaled gauge theory. We can simplify the sum over ordered partitions $\boldsymbol{h} \in \mathbb{N}_{0}^{4 h-2}$ of the integer $h$ by reducing it to a sum over partitions 
of $h$ into $m$ positive integers. By inserting 0 into all possible positions, we obtain $\left(\begin{array}{c}4 h-2 \\ m\end{array}\right)$ partitions of the original type in (6.17), and we find

$$
\begin{aligned}
\xi_{2 h-1}= & -\frac{\pi^{2 h-1}}{2 h-1} \sum_{m=1}^{h}\left(\begin{array}{c}
4 h-2 \\
m
\end{array}\right)\left(\frac{B_{0}}{\Gamma\left(\frac{3}{2}\right)}\right)^{4 h-2-m} \\
& \times \sum_{\substack{h \in \mathbb{N}^{m} \\
\sum_{k} h_{k}=h}}^{m} \frac{\left(2-2^{2 h_{k}}\right) B_{2 h_{k}}}{\left(2 h_{k}\right) ! \Gamma\left(\frac{3}{2}-2 h_{k}\right)} \\
= & -\sum_{m=1}^{h} \frac{(-1)^{m} 2^{2 h+m-1}(4 h-3) !}{(4 h-2-m) ! m !} \\
& \times \sum_{\substack{h \in \mathbb{N}^{m} \\
\sum_{k} h_{k}=h}}^{m} \frac{\left(2^{2 h_{k}-1}-1\right)\left(4 h_{k}-3\right) ! ! B_{2 h_{k}}}{\left(2 h_{k}\right) !}
\end{aligned}
$$

Finally, by exploiting the symmetry of the second summand in (6.18), we can reduce the sum over ordered partitions of $h$ with $m$ components to a sum over conjugacy classes and cycles of the symmetric group $S_{h}$, i.e., over unordered partitions of $h$. An unordered partition of $h$ is specified by $h$ non-negative integers $\nu_{k}$ with $\sum_{k} k \nu_{k}=h$, while the condition that the partition contain only $m$ parts is implemented by requiring that $\sum_{k} \nu_{k}=m$. By inserting the combinatorial factor $m ! / \nu_{1} ! \cdots \nu_{h}$ ! which counts the number of different ordered partitions that originate from the same unordered partition, we may bring (6.18) into our final equivalent form.

Theorem 2. The coefficients of the asymptotic expansion as $\mu \rightarrow \infty$ for the free energy ( 6.6) of $U(N)$ gauge theory on $\mathbb{T}^{2}$ in the double-scaling limit are given by

$$
\begin{aligned}
\xi_{2 h-1}= & (4 h-3) ! \sum_{m=1}^{h} \frac{(-1)^{m-1} 2^{m+2 h-1}}{(4 h-2-m) !} \\
& \times \sum_{\substack{\boldsymbol{\nu} \in \mathbb{N}_{0}^{h} \\
\sum_{k} k \nu_{k}=h, \sum_{k} \nu_{k}=m}} \prod_{k=1}^{h} \frac{1}{\nu_{k} !}\left(\frac{\left(2^{2 k-1}-1\right)(4 k-3) ! ! B_{2 k}}{(2 k) !}\right)^{\nu_{k}} .
\end{aligned}
$$


The rationality of these numbers will be important for the geometric interpretation of Section 6.3.

\subsection{Asymptotics of simple Hurwitz numbers}

We will now elucidate the geometrical meaning of the rational numbers (6.9) in order to pave the way towards the string interpretation of the doublescaling limit. For this, let us return to the chiral expansion (5.8) and (5.9) of the gauge theory in the ordinary 't Hooft limit. The $\lambda \rightarrow 0$ behaviour of the series (5.9) is controlled by the large $n$ asymptotics of the simple Hurwitz numbers $\sigma_{h}^{n}$ defined by (5.7) and (5.10), which geometrically count the number of holomorphic maps from a closed oriented Riemann surface of genus $h$ to the torus $\mathbb{T}^{2}$ with winding number $n$ and $2 h-2$ simple branch points. The first thing to realize is that, at fixed genus $h$, the $1 / \lambda$-type singularities of the free energy (5.9) as $\lambda \rightarrow 0$ are related to a power-like growth

$$
\sigma_{h}^{n} \simeq \beta_{h} n^{\alpha_{h}}
$$

of the number of covering maps with large winding number $n$, where $\alpha_{h}, \beta_{h}>$ 0 . The leading singularity of the series (5.8) and (5.9) as $\lambda \rightarrow 0$ is extracted by substituting (6.19) to get

$$
\begin{aligned}
\lim _{\lambda \rightarrow 0} F_{U(N)}^{+}(\lambda) & =\sum_{h=1}^{\infty}\left(\frac{2 \lambda}{N}\right)^{2 h-2} \beta_{h} \sum_{n=1}^{\infty} n^{\alpha_{h}} \mathrm{e}^{-n \lambda} \\
& =\sum_{h=1}^{\infty}\left(\frac{2 \lambda}{N}\right)^{2 h-2} \beta_{h} \operatorname{Li}_{-\alpha_{h}}\left(\mathrm{e}^{-\lambda}\right) .
\end{aligned}
$$

In the limit $\lambda \rightarrow 0$, we can substitute the singular behaviour of the Jonquière function $\mathrm{Li}_{-\alpha_{h}}(z)$ for $z \rightarrow 1^{-}$(see Appendix A., equation (A.5)) and to leading order we find

$$
\lim _{\lambda \rightarrow 0} F_{U(N)}^{+}(\lambda)=\sum_{h=1}^{\infty} \frac{2^{2 h-2} \beta_{h} \Gamma\left(\alpha_{h}+1\right)}{N^{2 h-2}} \lambda^{2 h-3-\alpha_{h}} .
$$

In the double-scaling limit, we insert (6.1) into (6.21). Comparing this with the weak-coupling expansion (6.8) of the free energy, we find the power 
of the growth (6.19) as the natural number

$$
\alpha_{h}=4 h-4,
$$

while the positive numbers $\beta_{h}$ are given by

$$
\beta_{h}=\frac{r_{4 h-3, h} \pi^{2 h}}{2^{2 h-2}(4 h-4) !}=\frac{\xi_{2 h-1} \pi^{2 h}}{2^{2 h-2}(4 h-3) !} .
$$

From the elementary power series identity

$$
\frac{1}{1-\mathrm{e}^{-\lambda}} \sum_{n=1}^{\infty} \sigma_{h}^{n} \mathrm{e}^{-n \lambda}=\sum_{n=1}^{\infty} \mathrm{e}^{-n \lambda} \sum_{k=1}^{n} \sigma_{h}^{k},
$$

we also obtain the simple relation

$$
\beta_{h}=(4 h-3) \lim _{M \rightarrow \infty} \frac{1}{M^{4 h-3}} \sum_{n=1}^{M} \sigma_{h}^{n},
$$

which will be crucial in the next section. That the explicit knowledge of the small area behaviour of the gauge theory allows one to reconstruct the asymptotic forms of the simple Hurwitz numbers is our first main geometric characterization.

Proposition 1. The asymptotic expansion as $\mu \rightarrow \infty$ for the free energy (6.6) of $U(N)$ gauge theory on $\mathbb{T}^{2}$ in the double-scaling limit is the generating function for the asymptotic Hurwitz numbers with

$$
\lim _{n \rightarrow \infty} \sigma_{h}^{n}=\frac{\pi^{2 h}}{2^{2 h-2}(4 h-3) !} \xi_{2 h-1} n^{4 h-4} .
$$

We have thereby found that, in the strong-coupling expansion of the $U(N)$ gauge theory, the double-scaling limit of the zero-instanton sector picks up the contributions, genus by genus, of branched covering maps over $\mathbb{T}^{2}$ with infinite degree and it sums the asymptotic behaviour of the simple Hurwitz numbers $\sigma_{h}^{n}$. Thus the saddle-point equation for the zero-instanton double-scaling free energy solves the combinatorial problem of determining the asymptotics of Hurwitz numbers. Proposition 1 combined with Theorem 2 produces a formula which coincides precisely with Theorem 7.1 of [61], whereby the asymptotics of simple Hurwitz numbers are evaluated directly by involving combinatorial techniques. This computation, which follows from the standard free fermion representation of two-dimensional Yang-Mills theory, is sketched in Appendix B.. We stress that here the saddle-point equation provides a very efficient and much simpler method for extracting these numbers. 


\subsection{Principal moduli spaces of holomorphic differentials}

We will now demonstrate that the double-scaling limit of the $U(N)$ gauge theory on $\mathbb{T}^{2}$ is in fact, rather remarkably, related to the geometry of some very special moduli spaces [61-64], whose "integer lattice" points (and thus their volumes) are "counted" by the strong-coupling expansion coefficients of Section 6.1. Let $\mathcal{H}_{h}$ be the moduli space of (topological classes of) pairs $(\Sigma, \mathrm{d} \boldsymbol{u})$, where $\Sigma$ is a compact Riemann surface of genus $h$ and $\mathrm{d} \boldsymbol{u}$ is a holomorphic 1-form on $\Sigma$ with exactly $m=2 h-2$ simple zeroes. The zero locus of $\mathrm{d} \boldsymbol{u}$ is the divisor

$$
\Delta_{\mathrm{d} \boldsymbol{u}}=\sum_{i=1}^{2 h-2}\left[u_{i}\right] \in \operatorname{Pic}(\Sigma), \quad u_{i} \in \Sigma .
$$

We call $\mathcal{H}_{h}$ a principal moduli space of holomorphic differentials or a principal stratum. Usually the points $u_{i}$ are taken to be ordered (distinguishable), so that the generalizations to the cases of zeroes of higher orders is straightforward. However, in our simpler situation, this choice will produce a redundant combinatorial factor to be introduced in the analysis below. The space $\mathcal{H}_{h}$ admits a natural action of the group $\mathrm{GL}_{+}(2, \mathbb{R})$ of $2 \times 2$ real matrices of positive determinant acting as

$$
\left(\begin{array}{l}
\operatorname{Re}(\mathrm{d} \boldsymbol{u}) \\
\operatorname{Im}(\mathrm{d} \boldsymbol{u})
\end{array}\right) \longmapsto\left(\begin{array}{ll}
a & b \\
c & d
\end{array}\right)\left(\begin{array}{l}
\operatorname{Re}(\mathrm{d} \boldsymbol{u}) \\
\operatorname{Im}(\mathrm{d} \boldsymbol{u})
\end{array}\right), \quad\left(\begin{array}{ll}
a & b \\
c & d
\end{array}\right) \in \mathrm{GL}_{+}(2, \mathbb{R})
$$

It may also be regarded as a fibration over the moduli space $\mathcal{M}_{h}$ of Riemann surfaces of genus $h$, with the fibre over $[\Sigma] \in \mathcal{M}_{h}$ equal to the vector space $\Gamma\left(\Sigma, \Omega^{1,0}(\Sigma)\right)$ modulo the action of the automorphism group $\operatorname{Aut}(\Sigma)$ of the curve.

We can coordinatize the moduli space $\mathcal{H}_{h}$ as follows. Consider the relative homology group $H_{1}\left(\Sigma, \Delta_{\mathrm{d} \boldsymbol{u}} ; \mathbb{Z}\right) \cong \mathbb{Z}^{4 h-3}$, and choose a basis of relative 1-cycles $\left\{\gamma_{i}\right\}_{i=1}^{4 h-3} \subset H_{1}\left(\Sigma, \Delta_{\mathrm{d} \boldsymbol{u}} ; \mathbb{Z}\right)$ such that $\gamma_{i}$ for $i=1, \ldots, 2 h$ form a canonical symplectic basis of 1-cycles for the ordinary homology group $H_{1}(\Sigma, \mathbb{Z})$, while the open contours $\gamma_{2 h+i}$ for $i=1, \ldots, 2 h-3$ connect the zeroes $u_{i+1}$ to $u_{1}$ on $\Sigma$, i.e., $\partial \gamma_{2 h+i}=\left[u_{i+1}\right]-\left[u_{1}\right]$. This basis may be conveniently chosen in such a way that each element is represented by a geodesic with respect to the flat metric $\operatorname{Re}(\mathrm{d} \boldsymbol{u} \overline{\mathrm{d} \boldsymbol{u}})$ induced by the holomorphic differential $\mathrm{d} \boldsymbol{u}$. This choice naturally cuts the surface into a union of flat polygons. We define the corresponding period map

$$
\phi: \mathcal{H}_{h} \longrightarrow \mathbb{C}^{4 h-3}
$$


by the formula

$$
\phi(\Sigma, \mathrm{d} \boldsymbol{u}):=\left(\oint_{\gamma_{1}} \mathrm{~d} \boldsymbol{u}, \ldots, \oint_{\gamma_{2 h}} \mathrm{~d} \boldsymbol{u}, \int_{\gamma_{2 h+1}} \mathrm{~d} \boldsymbol{u}, \ldots, \int_{\gamma_{4 h-3}} \mathrm{~d} \boldsymbol{u}\right)
$$

This map is holomorphic and locally injective, and so it defines a local system of complex coordinates on $\mathcal{H}_{h}$. This makes $\mathcal{H}_{h}$ a complex orbifold of dimension

$$
\operatorname{dim} \mathcal{H}_{h}=4 h-3
$$

The area $A_{\mathrm{d} \boldsymbol{u}}(\Sigma)$ of the surface $\Sigma$ with respect to the metric defined by the holomorphic 1-form d $\boldsymbol{u}$ can also be expressed in terms of the periods via the Riemann bilinear relation

$$
A_{\mathrm{d} \boldsymbol{u}}(\Sigma):=\frac{\mathrm{i}}{2} \int_{\Sigma} \mathrm{d} \boldsymbol{u} \wedge \overline{\mathrm{d} \boldsymbol{u}}=\frac{\mathrm{i}}{2} \sum_{i=1}^{h}\left(\oint_{\gamma_{i}} \mathrm{~d} \boldsymbol{u} \oint_{\gamma_{h+i}} \overline{\mathrm{d} \boldsymbol{u}}-\oint_{\gamma_{i}} \overline{\mathrm{d} \boldsymbol{u}} \oint_{\gamma_{h+i}} \mathrm{~d} \boldsymbol{u}\right)
$$

By using the period map, we can define a smooth measure on the moduli space $\mathcal{H}_{h}$ using the pull-back of the Lebesgue measure from $\mathbb{C}^{4 h-3}$ to $\mathcal{H}_{h}$ under $\phi$. We normalize this measure so that the volume of the unit cube in $\mathbb{C}^{4 h-3}$ is 1 . This measure is independent of the choice of basis $\left\{\gamma_{i}\right\}_{i=1}^{4 h-3}$. However, the total volume of $\mathcal{H}_{h}$ with respect to this measure is infinite. To cure this, we restrict to the subspace $\mathcal{H}_{h}^{\prime} \subset \mathcal{H}_{h}$ consisting of pairs $(\Sigma, \mathrm{d} \boldsymbol{u})$ of unit area $A_{\mathrm{d} \boldsymbol{u}}(\Sigma)=1$. This subset, which according to (6.31) defines a hyperboloid in $\mathcal{H}_{h}$ with respect to the coordinates chosen above, is invariant under the $\mathrm{SL}(2, \mathbb{R})$ subgroup of the $\mathrm{GL}_{+}(2, \mathbb{R})$ action $(6.27)$. The volume element $\operatorname{dvol}\left(\mathcal{H}_{h}\right)$ on $\mathcal{H}_{h}$ naturally induces a volume element $\operatorname{dvol}\left(\mathcal{H}_{h}^{\prime}\right)$ on the hypersurface $\mathcal{H}_{h}^{\prime}$ in the following way. Any element in $\mathcal{H}_{h}$ can be represented as $(\Sigma, r \mathrm{~d} \boldsymbol{u})$, where $r$ is a positive real number and $(\Sigma, \mathrm{d} \boldsymbol{u})$ represents an element of $\mathcal{H}_{h}^{\prime}$. We define the volume element $\operatorname{dvol}\left(\mathcal{H}_{h}^{\prime}\right)$ on the hyperboloid $\mathcal{H}_{h}^{\prime}$ by disintegration of the volume element $\operatorname{dvol}\left(\mathcal{H}_{h}\right)$ on $\mathcal{H}_{h}$ as

$$
\operatorname{dvol}\left(\mathcal{H}_{h}\right)=r^{4 h-4} \mathrm{~d} r \operatorname{dvol}\left(\mathcal{H}_{h}^{\prime}\right)
$$

It is an important result of ergodic theory that the volume of $\mathcal{H}_{h}^{\prime}$ is finite with respect to this measure $[65,66]$. In particular, the action of the diagonal subgroup of $\mathrm{SL}(2, \mathbb{R}) \subset \mathrm{GL}_{+}(2, \mathbb{R})$ on $\mathcal{H}_{h}^{\prime}$ is ergodic.

We will now explicitly compute the volumes of these moduli spaces. This can be done through an indirect procedure. One starts by introducing a 
sequence of cones

$$
\mathrm{C}_{M} \phi\left(\mathcal{H}_{h}^{\prime}\right):=\left\{t \phi\left(\mathcal{H}_{h}^{\prime}\right) \mid 0 \leq t \leq \sqrt{M}\right\}
$$

over $\phi\left(\mathcal{H}_{h}^{\prime}\right)$ with vertex at the origin of $\mathbb{C}^{4 h-3}$. We express the volume of the moduli space $\mathcal{H}_{h}^{\prime}$ in terms of the volume of the unit cone by the formula

$$
\operatorname{vol}\left(\mathcal{H}_{h}^{\prime}\right):=(4 h-3) \operatorname{vol}_{\mathbb{C}^{4 h-3}}\left(\mathrm{C}_{1} \phi\left(\mathcal{H}_{h}^{\prime}\right)\right)=(4 h-3) \int_{\mathrm{C}_{1} \phi\left(\mathcal{H}_{h}^{\prime}\right)} \mathrm{d} \nu 1,
$$

where $\mathrm{d} \nu$ is the Lebesgue measure on $\mathbb{C}^{4 h-3}$. The normalization factor takes into account the integration over radial part in (6.32). Computing the volume of the unit cone is actually simpler, as one can use an old trick. Consider an integer lattice $\Lambda \cong \mathbb{Z}^{2(4 h-3)}$ inside $\mathbb{R}^{2(4 h-3)} \cong \mathbb{C}^{4 h-3}$ and count the intersections of this lattice with $\mathrm{C}_{M} \phi\left(\mathcal{H}_{h}^{\prime}\right)$ for $M$ large. Their cardinality scales as

$$
\left|\mathrm{C}_{M} \phi\left(\mathcal{H}_{h}^{\prime}\right) \cap \Lambda\right| \simeq M^{4 h-3} \operatorname{vol}_{\mathbb{C}^{4 h-3}}\left(\mathrm{C}_{1} \phi\left(\mathcal{H}_{h}^{\prime}\right)\right),
$$

and thus the asymptotic behaviour of the left-hand side of (6.35) yields the volume we are looking for.

The relationship between these principal moduli spaces and the doublescaling limit of two-dimensional Yang-Mills theory on the torus $\mathbb{T}^{2}$ emerges when we have to select and geometrically interpret the integer lattice $\Lambda$. Any point $(\Sigma, \mathrm{d} \boldsymbol{u}) \in \mathcal{H}_{h}$, whose first $2 h$ coordinates $\phi_{i}(\Sigma, \mathrm{d} \boldsymbol{u}), i=1, \ldots, 2 h$, are integral (i.e., $\left.\phi_{i}(\Sigma, \mathrm{d} \boldsymbol{u}) \in \mathbb{Z}^{2}\right)$, is in one-to-one correspondence with a covering of the torus with only simple branch points and degree given by the area of $\Sigma$ [61]. Consider a simple branched covering

$$
\varpi: \Sigma \longrightarrow \mathbb{T}^{2}
$$

of the torus by a Riemann surface of genus $h$, with simple ramification over distinct points $z_{1}, \ldots, z_{2 h-2} \in \mathbb{T}^{2}$. With $\mathrm{d} z$ denoting the canonical holomorphic differential on $\mathbb{T}^{2}$, one can use the pull-back under (6.36) to associate the point

$$
\left(\Sigma, \varpi^{*}(\mathrm{~d} z)\right) \in \mathcal{H}_{h}
$$

with simple zeroes $u_{i}=\varpi^{-1}\left(z_{i}\right)$ corresponding to the ramification points of the cover. The integration of $\varpi^{*}(\mathrm{~d} z)$ over the homology cycles of $\Sigma$ produces integer values. Conversely, given $(\Sigma, \mathrm{d} \boldsymbol{u}) \in \mathcal{H}_{h}$ we can define a covering map (6.36) by

$$
z=\varpi(u):=\int^{u} \mathrm{~d} \boldsymbol{u} \bmod \mathbb{Z}^{2},
$$

which is well defined since $\oint_{\gamma} \mathrm{d} \boldsymbol{u} \in \mathbb{Z}^{2}$ for any 1-cycle $\gamma \subset \Sigma$. The critical points of $\varpi$ are precisely the simple zeroes $u_{i}$ of the holomorphic differential $\mathrm{d} \boldsymbol{u}=\varpi^{*}(\mathrm{~d} z)$, and $\varpi$ thereby has simple ramification at $u_{i} \in \Sigma$. The degree of the covering map (6.38) is the area (6.31). We have thereby arrived at 
a one-to-one correspondence between simple branched covers of the torus, and hence terms in the chiral Gross-Taylor string expansion, and integral points in the principal moduli spaces of holomorphic differentials.

We will now show that the numbers (6.34) are computed by the strongcoupling saddle-point expansion of the gauge theory that we obtained in Section 6.1. As alluded to above, the basic idea is to use a "refined" version of (6.35). By definition, we have

$$
\operatorname{vol}\left(\mathcal{H}_{h}^{\prime}\right)=(4 h-3) \lim _{M \rightarrow \infty} \frac{1}{M^{4 h-3}}\left|\mathrm{C}_{M} \phi\left(\mathcal{H}_{h}^{\prime}\right) \cap\left(\mathbb{Z}^{2(4 h-3)}+\boldsymbol{b}\right)\right|
$$

where the vector $\boldsymbol{b}=\left(b_{i}\right) \in \mathbb{C}^{4 h-3}$ has components $b_{i} \in \mathbb{Z}^{2}$ for $i=1, \ldots, 2 h$, while $b_{i} \neq b_{j} \bmod \mathbb{Z}^{2}$ for $i, j>2 h$ with $i \neq j$ (this vector is inserted in conjunction with the quantization properties of the period map (6.29)). On the other hand, from the above correspondence it follows that each point of the intersection $\mathrm{C}_{M} \phi\left(\mathcal{H}_{h}^{\prime}\right) \cap\left(\mathbb{Z}^{2(4 h-3)}+\boldsymbol{b}\right)$ corresponds to a simple branched cover $\varpi$ of $\mathbb{T}^{2}$ with winding number $\leq M$, and thus the volume (6.39) may be computed via the asymptotics of simple Hurwitz numbers as

$$
\operatorname{vol}\left(\mathcal{H}_{h}^{\prime}\right)=(2 h-2) !(4 h-3) \lim _{M \rightarrow \infty} \frac{1}{M^{4 h-3}} \sum_{n=1}^{M} \sigma_{h}^{n} .
$$

The combinatorial factor $(2 h-2)$ ! accounts for the fact that the ramification points are considered indistinguishable in the counting of the coverings. Comparing (6.23) and (6.25), we thereby find that the volumes are completely determined in terms of the coefficients $\xi_{2 h-1}$ of the saddle-point solution to be

$$
\operatorname{vol}\left(\mathcal{H}_{h}^{\prime}\right)=\frac{2^{2-2 h}(2 h-2) !}{(4 h-3) !} \xi_{2 h-1} \pi^{2 h} .
$$

Using the formula (6.41) and the explicit expansion (6.7), the first few volumes can be readily computed and are summarized in Table 1 . (These agree with the values for low genus computed in [64].) Their general expressions are provided by Theorem 2. In particular, the saddle-point computation explicitly demonstrates the rationality property $\pi^{-2 h} \operatorname{vol}\left(\mathcal{H}_{h}^{\prime}\right) \in \mathbb{Q}[62,63]$ and gives a precise geometrical meaning to the rational numbers that we first encountered in the weak-coupling expansions of the previous section. We may summarize the geometric interpretation of the double-scaling limit as follows.

Proposition 2. The asymptotic expansion as $\mu \rightarrow \infty$ for the free energy of $U(N)$ gauge theory on $\mathbb{T}^{2}$ in the double-scaling limit is the generating 
function for the volumes of the principal moduli spaces of holomorphic differentials given by

$$
\widehat{\mathcal{F}}_{U(N)}(\mu)=N \sum_{h=1}^{\infty} \frac{2^{2 h-2}(4 h-4) !}{(2 h-2) !}\left(\frac{1}{\pi \mu}\right)^{2 h-1} \operatorname{vol}\left(\mathcal{H}_{h}^{\prime}\right)+\mathrm{O}\left(\mathrm{e}^{-\mu}\right) .
$$

\subsection{Open string interpretation}

At this stage, we can speculate on the string interpretation of the doublescaling limit of the gauge theory that we have constructed above, with the foresight of the connection to a fluxon theory on the non-commutative plane that we will describe in the next section. First of all, only the chiral part of the Gross-Taylor series appears to contribute in the double-scaling limit of commutative $U(N)$ gauge theory on $\mathbb{T}^{2}$. This is not surprising upon examination of the explicit form for the non-chiral part of the free energy presented in [48]. It is not difficult to check that non-chiral contributions are always subleading in the small area limit. (An alternative perspective on these non-chiral couplings has been presented recently in [67].) It is in complete harmony with the fact, discussed at the beginning of Section 4.2 , that any theory of fluxons is necessarily chiral. Alternatively, we may regard this fact as being the statement that the holomorphic and anti-holomorphic sectors are identified with one another, so that the expansion is in terms of open strings. Being a chiral series, the original strong-coupling expansion is given as a sum over all Young diagrams, labelling representations of the infinite unitary group $U(\infty)$, which is the gauge group of a non-commutative gauge theory. In the strong-coupling regime, the double-scaling limit involves the

Table 1: The normalized volumes of the principal moduli spaces of holomorphic differentials up to genus $h=6$

\begin{tabular}{lc}
\hline$h$ & $\operatorname{vol}\left(\mathcal{H}_{h}^{\prime}\right) / \pi^{2 h}$ \\
\hline 1 & $1 / 3$ \\
2 & $1 / 135$ \\
3 & $1 / 4860$ \\
4 & $377 / 67359600$ \\
5 & $23357 / 157621464000$ \\
6 & $16493303 / 42765855611248000$ \\
\hline
\end{tabular}


asymptotic Hurwitz numbers, of branched covering maps to $\mathbb{T}^{2}$ in the limit of infinite winding number, again reflecting the open nature of the string degrees of freedom. This latter feature also implies that the toroidal spacetime is effectively decompactified onto the plane in the double-scaling limit, corresponding to the equi-anharmonic limit $\tau \rightarrow \mathrm{i} \infty$ of the underlying elliptic curve. This is certainly true of the limit analysed in Section 4 and is responsible for the loss of modular structure in the free energy (6.8) (see table 1).

Let us now note that the Gross-Taylor series is an expansion in $1 / N$, i.e., it is perturbative in the string coupling $g_{\mathrm{s}}$, and as such it ignores nonperturbative corrections of the form $\mathrm{e}^{-N \lambda}$. From (5.11), we see that these contributions have a natural interpretation [68] (see also [69]) as coming from (Euclidean) D1-branes of tension

$$
T_{1}=\frac{1}{\pi \alpha^{\prime} g_{\mathrm{s}}}
$$

which wrap around the target space torus $\mathbb{T}^{2}$ without foldings. There are no stable D0-branes in this picture [68], and so the non-perturbative structure is typical of a Type 0B string theory. Clearly, any consistent treatment of the new double-scaling limit should be able to capture the D-string contributions which are of order $\mathrm{e}^{-\mu}$, with the double-scaled coupling constant

$$
\mu=\frac{T_{1} A}{2 \pi}
$$

related to the brane tension. The free energy (6.4) computed from the exact solution of the saddle-point equation encodes contributions of this type. They are the constituents of the exponentially suppressed part at strong coupling in (6.6) and they appear consistently resummed by the saddlepoint equation. Alternatively, the necessary presence of these contributions can be understood from the point of view of the strong-coupling expansion. The series (6.6) truly diverges from the string theory perspective, i.e., in the double-scaling limit of the Gross-Taylor expansion, because the coefficients of the series grow as $(4 k-4) ! \Pi(k)$. It is unlikely that this series is even Borel summable, since $\Pi(k) \simeq \mathrm{e}^{\alpha \sqrt{k}}$ for large $k$ (cf. equation (3.14)). Instead, in our saddle-point approach this is just an artifact of the strongcoupling approximation, the exact solution being completely regular except at $\mu=0$ where the Douglas-Kazakov-type phase transition could take place (cf. figure 4). Thus the exponentially suppressed contributions of order $\mathrm{e}^{-\mu}$, interpreted in the string theory picture as contributions of D-strings, have to come into the game in order to ensure regularity of the complete 
result. It would be interesting to have a direct physical understanding in the field theory for the necessity of these non-perturbative D1-brane corrections.

These corrections will be particularly important in the non-commutative gauge theory because they will be enhanced in the required double-scaling limit of the $\mathrm{SU}(N) / \mathbb{Z}_{N}$ theory. The problem of computing the non-commutative free energy will thereby boil down to the problem of resumming these open string degrees of freedom. We expect that the D-strings which emerge in this way are the remnants of the electric dipole configurations in the instanton expansion.

With this line of reasoning, it is now tempting to conjecture a more direct open string interpretation of the expansion (6.8). In order to select the relevant geometrical structures, we start by rewriting the strong-coupling limit of the free energy (6.8) in terms of open string parameters as

$$
\mathcal{Z}_{\text {str }}\left(g_{\mathrm{s}}, T_{1} A\right):=\lim _{\mu \rightarrow \infty} \widehat{\mathcal{F}}_{U(N)}(\mu)=\frac{1}{g_{\mathrm{s}}} \sum_{k=1}^{\infty} \frac{2^{4 k-3}(4 k-3) !}{(2 k-2) !} \frac{\operatorname{vol}\left(\mathcal{H}_{k}^{\prime}\right)}{\left(T_{1} A\right)^{2 k-1}}
$$

We interpret this expansion as the remnant of a resummation of the D1branes. The effective action (6.44) is of order $1 / g_{\mathrm{s}}$, and it thereby represents an open string disk amplitude. Thus the double-scaling limit of commutative $U(N)$ gauge theory on $\mathbb{T}^{2}$, in the strong-coupling regime, can be interpreted as a theory of D1-branes at tree-level in open string perturbation theory. The truncation of the dynamics to tree level is presumably related to the fact that the strong-coupling regime of the double-scaling gauge theory is described by some sort of topological open string theory. In the next section, similar considerations in the $\mathrm{SU}(N) / \mathbb{Z}_{N}$ gauge theory, appropriate to the description of non-commutative Yang-Mills theory, will be used to argue that the fluxon expansion on $\mathbb{R}_{\Theta}^{2}$ is a certain non-commutative deformation of the open string theory described here.

Denoting by $\mathcal{H}^{\prime}:=\coprod_{h \in \mathbb{N}} \mathcal{H}_{h}^{\prime}$ the total moduli space of holomorphic differentials, we may write the string partition function (6.44) as the moduli space integral

$$
\mathcal{Z}_{\mathrm{str}}\left(g_{\mathrm{s}}, T_{1} A\right)=\int_{\mathcal{H}^{\prime}} \mathrm{dvol}_{\mathcal{H}^{\prime}}(\phi) W\left(\phi ; g_{\mathrm{s}}, T_{1} A\right)
$$

where the density $W\left(\phi ; g_{\mathrm{s}}, T_{1} A\right)$ is given by appropriate symmetry factors. From the general principles of topological string theory [55], we expect that this large area result computes the orbifold Euler characteristic $\chi\left(\mathcal{H}^{\prime}\right)$ of the moduli space $\mathcal{H}^{\prime}$. We can present some evidence in favour of this as follows. 
The moduli space $\mathcal{H}_{h}$ is connected [63] and can be related to $\mathcal{H}_{h}^{\prime}$ through the fibration

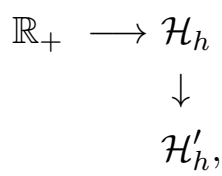

where the multiplicative group $\mathbb{R}_{+}=(0, \infty)$ is identified with the 1-parameter subgroup $\left\{\mathrm{e}^{t} \mathbb{1}_{2} \mid t \in \mathbb{R}\right\} \subset \mathrm{GL}_{+}(2, \mathbb{R})$ acting as in (6.27). Since the Euler character of a fibre bundle with connected base is the product of the Euler characters of the base and fibre, by taking $\chi\left(\mathbb{R}_{+}\right)=1$ we find the equality

$$
\chi\left(\mathcal{H}_{h}\right)=\chi\left(\mathcal{H}_{h}^{\prime}\right)
$$

Next we apply the fibration noted in the previous section,

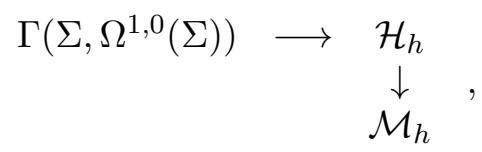

along with the Harer-Zagier-Penner formula [70] for the Euler characters of Riemann moduli spaces to compute

$$
\chi\left(\mathcal{H}_{h}^{\prime}\right)=\chi\left(\mathcal{M}_{h}\right) \chi\left[\Gamma\left(\Sigma, \Omega^{1,0}(\Sigma)\right)\right]=\frac{B_{2 h}}{2 h(2 h-2)} \chi\left[\Gamma\left(\Sigma, \Omega^{1,0}(\Sigma)\right)\right] .
$$

Given the explicit formulas for the $\operatorname{volumes} \operatorname{vol}\left(\mathcal{H}_{h}^{\prime}\right)$ obtained in this section, relation (6.49) strongly suggests that an explicit realization of the string partition function (6.45) as an Euler sigma-model of the form $\mathcal{Z}_{\mathrm{str}} \simeq \chi\left(\mathcal{H}^{\prime}\right)=$ $\int_{\mathcal{H}^{\prime}} e\left(T \mathcal{H}^{\prime} \rightarrow \mathcal{H}^{\prime}\right)$ should hold, with $e\left(T \mathcal{H}^{\prime} \rightarrow \mathcal{H}^{\prime}\right)$ the Euler characteristic class of the tangent bundle over the moduli space $\mathcal{H}_{h}^{\prime}$. This would directly connect the double-scaling string theory with a certain topological string theory [55]. We may also expect the form (6.45) to be generically valid at finite area, so that the string path integral localizes in the usual way onto finite-dimensional moduli spaces. The double-scaling limit replaces the Hurwitz moduli space of simple branched covers of $\mathbb{T}^{2}$, arising in the 't Hooft limit of $\mathrm{QCD}_{2}$ on the torus [55], by the principal moduli space of holomorphic differentials.

This realization is the starting point for an investigation of the rewriting of the double-scaling expansion as some sort of topological field theory. The action principle for this open string theory is given by the topologically twisted $\mathcal{N}=2$ superconformal field theory coupled to gravity that describes the closed string expansion of two-dimensional Yang-Mills theory [54], by taking the torus target space to be equi-anharmonic after a modular transformation. The specification of vacua in this context requires a choice of holomorphic vector bundle over the underlying elliptic curve, and the 1-loop 
open string partition function is computed as the corresponding holomorphic Ray-Singer analytic torsion, represented concisely in terms of an index integral as in the closed string case. This would thus identify the open string action in terms of a deformed topological sigma-model coupled to topological gravity. The double-scaling limit replaces the counting of holomorphic maps $\Sigma \rightarrow \mathbb{T}^{2}$, arising in the 't Hooft limit of $\mathrm{QCD}_{2}$ on the torus [54], by the counting of holomorphic differentials on complex curves $\Sigma$. This may be loosely interpreted as replacing a topological A-model string theory with elliptic curve target space by a topological B-model string theory. An alternative action principle for the ordinary $\mathrm{QCD}_{2}$ string is proposed in [71].

The relation between two-dimensional Yang-Mills theory and string theory has been exploited recently in connection with $\mathcal{N}=2$ supersymmetric gauge theories in four dimensions [72]. The large $N$ limit of the partition function of $U(N)$ Yang-Mills theory on the sphere $\mathbb{S}^{2}$ reproduces the instanton counting of four-dimensional $\mathcal{N}=2$ supersymmetric gauge theories introduced in $[73,74]$. The relevant instanton moduli spaces, appropriate for the counting, have also been shown to map onto the moduli spaces of punctured Riemann spheres [75], suggesting a relation between instantons in four dimensions and Hurwitz numbers. Because the rationale behind this circle of ideas is still topological string theory, it could be interesting to explore the connection of our construction with these recent results.

We can now argue that the indications of a string theory that we have found are consistent, at least at a qualitative level, with the spectrum of instanton strings that we found in Section 3. First of all, the moduli spaces $\mathcal{H}_{h}^{\prime}$ admit an ergodic action of the group $\mathrm{SL}(2, \mathbb{R})$. This action is the Teichmüller geodesic flow which gives the Euler-Lagrange equations for geodesics with respect to the Teichmüller metric on the moduli space $\mathcal{M}_{h}$ of complex curves of genus $h$ [62]. The chaotic nature of these flows on the moduli space $\mathcal{H}^{\prime}$ suggests that the topological string theory amplitudes (6.45) may admit an interpretation as computing certain fractal dimensions, tied to the structure of the orbifold Euler characteristics described above. This property may be related to the fractal characteristics of the non-commutative instanton spectrum that we conjectured in Section 3. Second, the free fermion formalism described in Appendix B. shows that the string states which contribute to the string expansion of the gauge theory are represented by the partition basis (B.6) of the fermionic Fock space. In the double-scaling limit, strings of very large winding number dominate and are represented by very large partitions $\boldsymbol{n}$, i.e., $\sum_{l} n_{l}=n$ with $n \rightarrow \infty$. From the Hardy-Ramanujan formula (3.14), it follows that the degeneracy of such string states grows like $\Pi(n) \simeq \mathrm{e}^{\alpha \sqrt{n}}$, producing the familiar 
exponential form of worldsheet densities of states. This is completely analogous to the asymptotic behaviour of the instanton density of states that we described in Section 3.

We should also explore the possibility that a Douglas-Kazakov-type phase transition could take place for some finite value of the coupling $\mu>0$ in the double-scaling limit, in contrast to what occurs in the ordinary 't Hooft limit. Note that this limit necessarily restricts us to the weak-coupling regime of the gauge theory, in which only the instanton expansion is available. To answer the question of a non-trivial phase transition requires an accurate analysis of all higher instanton contributions to the partition function and one must really face up to the entire instanton series.

\section{Non-commutative instanton string representation}

In this section, we will examine the instanton expansion of non-commutative Yang-Mills theory on $\mathbb{T}^{2}$ with rational-valued non-commutativity parameter $\theta=1 / N(n=1$ in the notations of Section 4.1). By Morita equivalence, this theory is completely identical to commutative $U(N)$ gauge theory on $\mathbb{T}^{2}$ in a certain Chern class $q \in \mathbb{Z}$. Because of the global group isomorphism $U(N) \cong U(1) \times \mathrm{SU}(N) / \mathbb{Z}_{N}$, the partition function of this theory receives a trivial contribution $\exp \left(-2 \pi^{2} q^{2} / g^{2} A N\right)$ from the $U(1)$ sector, which is exactly cancelled by the background gauge field generated in the Morita transformation $[9,25]$. The remainder is the partition function of $\mathrm{SU}(N) / \mathbb{Z}_{N}$ gauge theory on $\mathbb{T}^{2}$ with Chern number $q$. The Chern classes are labelled by elements of the fundamental group of the gauge group, which in the present case is

$$
\pi_{1}\left(\frac{\mathrm{SU}(N)}{\mathbb{Z}_{N}}\right)=\pi_{0}\left(\mathbb{Z}_{N}\right)=\mathbb{Z}_{N}
$$

Thus there are $N$ topological 't Hooft flux sectors labelled by $q=0,1, \ldots$, $N-1$ [42]. The Morita transformation also shrinks the area of the torus by a factor of $N^{2}, A \rightarrow A / N^{2}$, and rescales the Yang-Mills coupling constant as $g^{2} \rightarrow g^{2} / N[9,25]$.

The large $N$ limit required to produce gauge theory on the non-commutative plane involves a double-scaling limit defined by taking $A \rightarrow \infty$ in (4.9) with $\Theta$ fixed and $n=1$. Because of the rescalings generated by Morita equivalence, it follows that it is precisely the coupling constant (6.1) which is kept finite in this limit, and it is given in terms of the parameters of the non-commutative gauge theory as

$$
\mu=g^{2} \Theta .
$$


It is possible to carry out a double-scaling limit in which the size $\sqrt{A}$ of the torus is also kept finite in the large $N$ limit [16], by fixing instead the 't Hooft coupling constant (5.2) and sending $n \rightarrow \infty$ in (4.9) with $n / N$ fixed, but here we shall deal only with the somewhat simpler case where the limiting gauge theory lives on $\mathbb{R}_{\Theta}^{2}$ rather than on $\mathbb{T}_{\theta}^{2}$.

In this section, we will analyse the non-commutative gauge theory by generalizing the technique of the previous section and use it to describe a possible open string representation for gauge theory on the non-commutative plane $\mathbb{R}_{\Theta}^{2}$. We will attempt to interpret this string theory, in the strongcoupling regime of the gauge theory, as a tree-level theory of D1-branes similar to the one described in Section 6.4. This analysis may shed light on the nature of the full string theory that reproduces the spectrum of noncommutative instantons that we analysed in detail in Section 3. Heuristically, because $\mathrm{SU}(N)$ Yang-Mills theory on $\mathbb{T}^{2}$ is a string theory at large $N$, it induces a corresponding string representation of the non-commutative gauge theory obtained through the double scaling limit described above. We will find that this gives a certain closed gauge string theory description of the origin of fluxons on the non-commutative plane.

\subsection{Instanton expansion of $\mathrm{SU}(N) / \mathbb{Z}_{N}$ gauge theory}

We begin by rewriting the the instanton representation of commutative $\mathrm{SU}(N) / \mathbb{Z}_{N}$ gauge theory on $\mathbb{T}^{2}$, in the $q$ th 't Hooft sector, in a manner analogous to what we did for the $U(N)$ theory in Section 5.2. The partition function can be read off from the general instanton series (2.12) at $\theta=0$, $p=N-1$ by inserting the appropriate background flux shifts in the Chern numbers and the appropriate terms which arise from the Migdal expansion. It is given explicitly by $[12,25]$

$$
\begin{aligned}
Z_{\mathrm{SU}(N)}^{(q)}\left(g^{2} A\right)= & (-1)^{N+(N-1) q} \mathrm{e}^{-\varepsilon_{\mathrm{F}}}\left\{\sqrt{\frac{2 \pi^{2}}{g^{2} A N}}+\sum_{\substack{\boldsymbol{\nu} \in \mathbb{N}_{0}^{N-1} \\
\sum_{k} k \nu_{k}=N}}^{\prime} \prod_{k=1}^{N-1} \frac{(-1)^{\nu_{k}}}{\nu_{k} !}\right. \\
& \times\left(\frac{2 \pi^{2}}{k^{3} g^{2} A}\right)^{\nu_{k} / 2} \sum_{\substack{\boldsymbol{q} \in \mathbb{Z}^{|\boldsymbol{\nu}|} \\
\sum_{j} q_{j}=q}} \exp \left[-\frac{2 \pi^{2}}{g^{2} A} \sum_{l=1}^{N} \frac{1}{l} \sum_{j=1+\nu_{1}+\cdots+\nu_{l-1}}^{\nu_{1}+\cdots+\nu_{l}}\right. \\
& \left.\left.\times\left(q_{j}-\frac{l q}{N}\right)^{2}\right]\right\}
\end{aligned}
$$


where we use the same conventions as in (5.22). The prime on the first sum in (7.3) means that it omits the trivial partition of $N$ which has $\nu_{1}=N, \nu_{k}=$ $0 \forall k>1$. This is the contribution from vacuum fluctuations represented by the first non-exponential term in (7.3), and it is thereby associated with the contribution from the zero-instanton sector of the theory. The infinite series then contains the contributions from all multi-instanton configurations.

We proceed as in Section 5.2, except that we now introduce a double contour integration to resolve both the partition and topological charge constraints in (7.3). One finds

$$
\begin{aligned}
Z_{\mathrm{SU}(N)}^{(q)}\left(g^{2} A\right)= & (-1)^{N} \mathrm{e}^{-\varepsilon_{\mathrm{F}}+\left(2 \pi^{2} q^{2} / g^{2} A N\right)} \oint_{\mathcal{C}_{0}(z)} \frac{\mathrm{d} z}{2 \pi \mathrm{i} z^{N+1}} \oint_{\mathcal{C}_{0}(w)} \frac{\mathrm{d} w}{2 \pi \mathrm{i} w^{q+1}} \\
& \times \sum_{\nu \in \mathbb{N}_{0}^{N-1}} \prod_{k=1}^{N-1} \frac{(-1)^{\nu_{k}}}{\nu_{k} !}\left(\frac{2 \pi^{2}}{k^{3} g^{2} A}\right)^{\nu_{k} / 2} z^{k \nu_{k}}\left[\Xi_{k}\left(w ; g^{2} A\right)\right]^{\nu_{k}}
\end{aligned}
$$

where we have absorbed the identity contribution to (7.3) into the sum over all $\boldsymbol{\nu} \in \mathbb{N}_{0}^{N-1}$ in (7.4), and defined the sequence of functions

$$
\Xi_{k}\left(w ; g^{2} A\right):=\sum_{m=-\infty}^{\infty}(-1)^{(N-1) m} w^{m} \mathrm{e}^{-2 \pi^{2} / g^{2} A m^{2} / k}, \quad k=1, \ldots, N-1,
$$

which coincide with (5.23) at $w=1$. Carrying out the sums in (7.4) thereby leads to the formula

$$
\begin{aligned}
Z_{\mathrm{SU}(N)}^{(q)}\left(g^{2} A\right)= & \oint_{\mathcal{C}_{0}(z)} \frac{\mathrm{d} z}{2 \pi \mathrm{i} z^{N+1}} \oint_{\mathcal{C}_{0}(w)} \frac{\mathrm{d} w}{2 \pi \mathrm{i} w^{q+1}} \exp \\
& \times\left[-\sqrt{\frac{2 \pi^{2}}{g^{2} A}} \sum_{k=1}^{\infty} \Xi_{k}\left(w ; g^{2} A\right) \frac{(-z)^{k}}{k^{3 / 2}}\right] \mathrm{e}^{-\varepsilon_{\mathrm{F}}+2 \pi^{2} q^{2} / g^{2} A N}
\end{aligned}
$$

By changing integration variables $z \rightarrow z / w$ and then rescaling $z \rightarrow \mathrm{e}^{2 \pi^{2} / g^{2} A} z$, $w \rightarrow \mathrm{e}^{4 \pi^{2} / g^{2} A} w$, one finds that the partition function (7.6) depends only on the value of the 't Hooft flux $q$ modulo $N$,

$$
Z_{\mathrm{SU}(N)}^{(q+N)}\left(g^{2} A\right)=Z_{\mathrm{SU}(N)}^{(q)}\left(g^{2} A\right)
$$

as it should.

Let us now compare the $\mathrm{SU}(N) / \mathbb{Z}_{N}$ gauge theory with the strong-coupling expansion of the $U(N)$ gauge theory that we obtained in Section 5.2. Following the steps which led to (5.29), the only changes in the present case are that the Fermi level (5.28) is shifted as $n_{\mathrm{F}} \rightarrow n_{\mathrm{F}}+\ln (w) / 2 \pi \mathrm{i}$ and 
the Fermi energy (5.19) by the $U(1)$ charge contribution of the $q$ th flux sector, along with the accompanying contour integration over $\mathcal{C}_{0}(w)$. In this way, we arrive at the strong-coupling form

$$
\begin{aligned}
Z_{\mathrm{SU}(N)}^{(q)}\left(g^{2} A\right)= & \oint_{\mathcal{C}_{0}(z)} \frac{\mathrm{d} z}{2 \pi \mathrm{i} z^{N+1}} \oint_{\mathcal{C}_{0}(w)} \frac{\mathrm{d} w}{2 \pi \mathrm{i} w^{q+1}} \prod_{n=-\infty}^{\infty} \\
& \times\left(1+z \mathrm{e}^{-\left(g^{2} A / 2\right)\left(n-n_{\mathrm{F}}-\ln w / 2 \pi \mathrm{i}\right)^{2}}\right) \mathrm{e}^{-\varepsilon_{\mathrm{F}}+2 \pi^{2} q^{2} / g^{2} A N} .
\end{aligned}
$$

Again, the Migdal expansion is recovered by expanding the product and doing the integrals, with the contour integration over $w$ implementing the appropriate $U(1)$ charge subtraction in the $q$ th topological sector. In terms of the Young tableaux variables of Section 5.1, the 't Hooft fluxes are given by $N q=\sum_{k} n_{k}-n$, while the quadratic Casimir (5.3) is shifted as $C_{2}(R(Y)) \rightarrow C_{2}(R(Y))-(N q+n)^{2} / N$ for $Y \in \mathcal{Y}_{n}$. The ordinary $\operatorname{SU}(N)$ partition function is given by (7.8) in the trivial sector $q=0$ with $Z_{\mathrm{SU}(N)}$ $\left(g^{2} A\right)=N Z_{\mathrm{SU}(N)}^{(0)}\left(g^{2} A\right)$. On the other hand, the $U(N)$ partition function is recovered by reinstating the $U(1)$ charge subtraction and summing $\exp \left(-2 \pi^{2} q^{2} / g^{2} A N\right) Z_{\mathrm{SU}(N)}^{(q)}\left(g^{2} A\right)$ over all $q \in \mathbb{N}_{0}$. Using the identity $\sum_{q \in \mathbb{N}_{0}}$ $w^{-q}=w /(w-1)$ and evaluating the residue of the simple pole arising in (7.8) at $w=1$, we arrive at (5.29).

\subsection{Double-scaling limit}

Starting from the contour integral representation (7.6), we shall now study the large $N$ double-scaling limit of the $\mathrm{SU}(N) / \mathbb{Z}_{N}$ gauge theory in which the coupling constant (6.1) and (7.2) is held fixed. In this limit, we reproduce non-commutative gauge theory in two dimensions. Let us assume for simplicity that the rank $N$ is odd, so that the Fermi level (5.28) is an integer. This assumption has no bearing on the final results obtained in the $N \rightarrow \infty$ limit. We also rescale the integration variable $w \rightarrow \mathrm{e}^{N \pi q / g^{2} \Theta} w$ and thereby write (7.6) in the form

$$
\begin{aligned}
\widehat{\mathcal{Z}}_{\mathrm{SU}(N)}^{(q)}\left(g^{2} \Theta\right)= & \mathrm{e}^{\pi N g^{2} \Theta / 12} \oint_{\mathcal{C}_{0}(z)} \frac{\mathrm{d} z}{2 \pi \mathrm{i} z^{N+1}} \exp \left[-N \sqrt{\frac{\pi}{g^{2} \Theta}} \operatorname{Li}_{3 / 2}(-z)\right] \\
& \times \oint_{\mathcal{C}_{0}(w)} \frac{\mathrm{d} w}{2 \pi \mathrm{i} w^{q+1}} \exp \left[-N \sqrt{\frac{\pi}{g^{2} \Theta}} \sum_{k=1}^{\infty} \frac{(-z)^{k}}{k^{3 / 2}} \sum_{m=1}^{\infty}\right. \\
& \left.\times \mathrm{e}^{-\left(N^{2} \pi / g^{2} \Theta\right) m^{2} / k}\left(\mathrm{e}^{N \pi q m / g^{2} \Theta} w^{m}+\mathrm{e}^{-N \pi q m / g^{2} \Theta} w^{-m}\right)\right] .
\end{aligned}
$$


This form of the partition function exhibits it as a factorization into the zeroinstanton vacuum amplitude of the $U(N)$ theory that we encountered in the previous section, plus two infinite series comprising all the positively and negatively charged higher instanton contributions. When the contour $\mathcal{C}_{0}(w)$ is the unit circle we have $w^{-1}=\bar{w}$, suggesting a sort of chiral/anti-chiral factorization for the instanton contributions.

With respect to the zero-instanton sector, we can single out those higher instanton configurations which yield contributions that are not exponentially suppressed in $N$. The positive and negative instanton contributions in (7.9) are weighted, respectively, by Boltzmann factors with the modified actions

$$
S_{\text {inst }}^{( \pm)}\left(m, k ; g^{2} \Theta\right):=\frac{N \pi q m}{g^{2} \Theta}\left(\frac{N m}{q k} \mp 1\right) .
$$

By using the $\mathbb{Z}_{N}$ symmetry (7.7) of the partition function, we can choose the flux $q$ to lie in the range $-n_{\mathrm{F}} \leq q \leq n_{\mathrm{F}}$ without loss of generality. Suppose first that $q>0$. Then the solutions of the Diophantine equation

$$
N m-q k=n_{+},
$$

with $n_{+} \in \mathbb{N}$ of order 1 in the large $N$ limit, select an infinite collection of pairs of integers $(m, k)$ for which the instanton action $S_{\text {inst }}^{(+)}\left(m, k ; g^{2} \Theta\right)$ is finite at $N \rightarrow \infty$. If $n_{+}<0$ then no finite solution is possible, because from (7.4) it follows that the large $N$ limit must be taken with $0<k<N$. In addition, the action $S_{\text {inst }}^{(-)}\left(m, k ; g^{2} \Theta\right)$ is infinite, because the analogous condition $N m+q k=n_{-} \in \mathbb{N}$ for it necessarily requires $n_{-}$to be of order $N$ due to the positivity of the integer $k$. From (7.11) it follows that the surviving action admits the $1 / N$ expansion

$$
S_{\text {inst }}^{(+)}\left(m, k ; g^{2} \Theta\right)=\frac{N \pi q m}{g^{2} \Theta} \frac{n_{+}}{q k}=\frac{\pi q n_{+}}{g^{2} \Theta} \frac{1}{1-n_{+} / N m}=\frac{\pi \ell}{g^{2} \Theta}+\mathrm{O}\left(\frac{1}{N}\right) .
$$

The leading non-vanishing term in (7.12) at $N=\infty$ is simply the action for a fluxon of charge $\ell=q n_{+}$. For $q<0$, it is instead the action $S_{\text {inst }}^{(-)}\left(m, k ; g^{2} \Theta\right)$ which survives the large $N$ double-scaling limit. Therefore, in a manner very similar to that of Section 4.1, the large $N$ double-scaling limit of the $\mathrm{SU}(N) / \mathbb{Z}_{N}$ gauge theory becomes dominated by the fluxon solutions of noncommutative gauge theory.

As discussed in Section 4, the limiting gauge theory on the non-commutative plane $\mathbb{R}_{\Theta}^{2}$ is independent of the choice of non-trivial projective module for the parent gauge theory on the torus $\mathbb{T}^{2}$, and hence we may restrict our attention to the flux sector $q=-1$ without loss of generality. We rewrite the partition function $\widehat{\mathcal{Z}}_{\infty}\left(g^{2}, \Theta\right):=\widehat{\mathcal{Z}}_{\mathrm{SU}(N)}^{(-1)}\left(g^{2} \Theta\right)$ in $(7.9)$ by replacing the 
sum over $k \in \mathbb{N}$ with a sum over the fluxon charge $\ell=N m-k$. Since $k \geq 1$, the charge is bounded from above and in the limit $N \rightarrow \infty$ one has

$$
\begin{aligned}
\left.\widehat{\mathcal{Z}}_{\infty} g^{2}, \Theta\right)= & \mathrm{e}^{\pi N g^{2} \Theta / 12} \oint_{\mathcal{C}_{0}(z)} \frac{\mathrm{d} z}{2 \pi \mathrm{i} z^{N+1}} \exp \left[-N \sqrt{\frac{\pi}{g^{2} \Theta}} \operatorname{Li}_{3 / 2}(-z)\right] \oint_{\mathcal{C}_{0}(w)} \\
& \times \frac{\mathrm{d} w}{2 \pi \mathrm{i}} \exp \left[-N \sqrt{\frac{\pi}{g^{2} \Theta}} \sum_{m=1}^{\infty} w^{-m} \sum_{\ell=-\infty}^{N m-1} \mathrm{e}^{-\pi \ell / g^{2} \Theta} \frac{(-z)^{N m-\ell}}{(N m-\ell)^{3 / 2}}\right]
\end{aligned}
$$

This expression demonstrates that the approximation we have employed keeps only the chiral part of the full instanton series, in the sense that only negative magnetic charges (i.e., negative powers of $w$ ) appear. This is in beautiful harmony with the chiral nature of the double-scaling limit that we discovered in the previous section, and also with the fact that any theory involving fluxons is necessarily chiral.

We now expand the factor $(N m-\ell)^{-3 / 2}$ in (7.13) for $\ell$ of order 1 in the large $N$ limit and rescale the second contour integration variable as $w \rightarrow z^{N} w$. We then expand the second exponential function and perform the integral over $w$ to arrive at

$$
\begin{aligned}
\widehat{\mathcal{Z}}_{\infty}\left(g^{2}, \Theta\right)= & \sum_{\ell=-\infty}^{N-1}(-1)^{\ell} \mathrm{e}^{-\pi \ell / g^{2} \Theta} \oint_{\mathcal{C}_{0}} \frac{\mathrm{d} z}{2 \pi \mathrm{i} z^{\ell+1}} \exp \left[-N \sqrt{\frac{\pi}{g^{2} \Theta}} \operatorname{Li}_{3 / 2}(-z)\right] \\
& \times \sqrt{\frac{\pi}{N g^{2} \Theta}} \mathrm{e}^{\pi N g^{2} \Theta / 12} .
\end{aligned}
$$

To sum the series in (7.14), we decompose the integration contour into two connected, closed components as $\mathcal{C}_{0}=\mathcal{C}_{0}^{+} \cup \mathcal{C}_{0}^{-}$. The contour $\mathcal{C}_{0}^{+}$is situated in the region $\mathrm{e}^{-\pi / g^{2} \Theta}<|z|<1$ of the complex $z$-plane and along it the sum over $\ell=0,1, \ldots, N-1$ can be performed, while the contour $\mathcal{C}_{0}^{-}$is located at $|z|<\mathrm{e}^{-\pi / g^{2} \Theta}$ and along it we may carry out the sum over all $\ell \leq-1$. After dropping the irrelevant multiplicative constant, we arrive in this way at the formula

$$
\begin{aligned}
\widehat{\mathcal{Z}}_{\infty}\left(g^{2}, \Theta\right)= & \mathrm{e}^{\pi N g^{2} \Theta / 12}\left\{\oint_{\mathcal{C}_{0}^{-}} \frac{\mathrm{d} z}{2 \pi \mathrm{i} z} \frac{z \mathrm{e}^{\pi / g^{2} \Theta}}{1+z \mathrm{e}^{\pi / g^{2} \Theta}} \exp \left[-N \sqrt{\frac{\pi}{g^{2} \Theta}} \operatorname{Li}_{3 / 2}(-z)\right]\right. \\
& \left.-\oint_{\mathcal{C}_{0}^{+}} \frac{\mathrm{d} z}{2 \pi \mathrm{i} z} \frac{1+\left(\mathrm{e}^{-\pi / g^{2} \Theta} / z\right)^{N}}{1+\mathrm{e}^{-\pi / g^{2} \Theta} / z} \exp \left[-N \sqrt{\frac{\pi}{g^{2} \Theta}} \operatorname{Li}_{3 / 2}(-z)\right]\right\} .
\end{aligned}
$$

Since the contour $\mathcal{C}_{0}^{-}$does not catch the pole at $z=-\mathrm{e}^{-\pi / g^{2} \Theta}$, the first integral in (7.15) vanishes by Cauchy's theorem. To evaluate the contribution of the second integral, we expand it into a sum of two contour integrals, the 
first one having a trivial dependence on $N$. Since by construction $\mathcal{C}_{0}^{+}$does encircle the pole, this first contribution can be safely evaluated by using the residue theorem.

By using the relations (4.9) and (7.2), we finally arrive in this way at the expression

$$
\begin{aligned}
\widehat{\mathcal{Z}}_{\infty}\left(g^{2}, \Theta\right)= & \mathrm{e}^{\pi N g^{2} \Theta / 12} \mathcal{Z}_{\infty}\left(g^{2}, \Theta\right)+\mathrm{e}^{\pi N\left(g^{2} \Theta / 12-1 / g^{2} \Theta\right)} \oint_{\mathcal{C}_{0}^{+}} \frac{\mathrm{d} z}{2 \pi \mathrm{i} z^{N+1}} \\
& \times \frac{z}{z+\mathrm{e}^{-\pi / g^{2} \Theta}} \exp \left[-N \sqrt{\frac{\pi}{g^{2} \Theta}} \operatorname{Li}_{3 / 2}(-z)\right]
\end{aligned}
$$

for the gauge theory partition function on the non-commutative plane $\mathbb{R}_{\Theta}^{2}$. The first term in (7.16) is exactly the resummation of the weak-coupling semi-classical expansion of the gauge theory in terms of fluxons, as obtained in (4.39). The second term is similar to the contribution of the $U(N)$ zeroinstanton sector that was studied in the previous section, with the important difference that in evaluating the saddle-point position there are corrections coming from the function $z /\left(z+\mathrm{e}^{-\pi / g^{2} \Theta}\right)$ occuring in the integrand. In the $1 / N$ expansion these contributions can be considered subleading and we may discard them in a first approximation. We can therefore identify, at leading order, the second contribution with the previously computed one in the $U(N)$ gauge theory, up to the relevant multiplicative factors. Note that the vacuum energy is not cancelled out in the fluxon contribution.

\subsection{Weak and strong coupling limits}

The two terms in the decomposition (7.16) represent, respectively, the contributions from the zero-instanton sector and the collective resummation of higher instanton sectors of the $\mathrm{SU}(N) / \mathbb{Z}_{N}$ gauge theory on $\mathbb{T}^{2}$ in the double-scaling limit. Since $\mathrm{Li}_{3 / 2}(0)=0$, in the weak-coupling limit $g^{2} \Theta \rightarrow 0$ the partition function (4.39) is of order 1 and thus the first term in (7.16) dominates the full vacuum amplitude. This is of course the regime wherein we anticipate the non-commutative instanton expansion to be valid. We can now analyse if there exists some finite value of the double-scaling coupling $\mu=g^{2} \Theta>0$ for which the second term in (7.16) dominates over the fluxon partition function. For this, we rewrite (7.16) in terms of the fluxon free energy $\mathcal{F}_{\infty}\left(g^{2}, \Theta\right)=\ln \mathcal{Z}_{\infty}\left(g^{2}, \Theta\right)$ as

$$
\widehat{\mathcal{Z}}_{\infty}\left(g^{2}, \Theta\right)=: \mathrm{e}^{\left(\pi N g^{2} \Theta / 12\right)+\mathcal{F}_{\infty}\left(g^{2}, \Theta\right)}\left(1+\mathrm{e}^{\Delta \mathcal{F}_{\infty}\left(g^{2}, \Theta\right)}\right),
$$

and seek regions in parameter space where the free energy fluctuation function $\Delta \mathcal{F}_{\infty}\left(g^{2}, \Theta\right)$ yields a dominant contribution to the partition function (7.17). The result of a numerical evaluation of this function is depicted in 


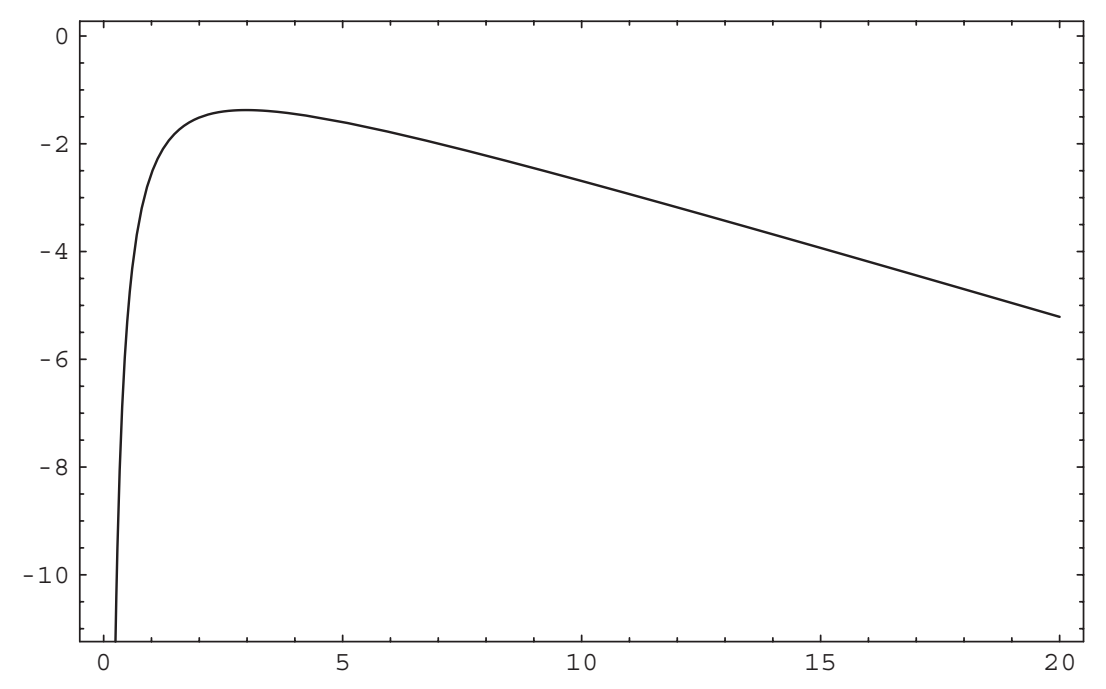

Figure 5: The zero-instanton free energy fluctuation $\Delta \mathcal{F}_{\infty}$ as a function of the double-scaling parameter $\mu=g^{2} \Theta$.

figure 5. We see that $\Delta \mathcal{F}_{\infty}\left(g^{2}, \Theta\right)<0$ over the full range of parameters and thus the corrections to the fluxon contribution in (7.16) are exponentially suppressed in the double-scaling limit. Thus the phase transition described earlier, potentially driven by the enhancement of the collective higher instanton configurations, does not occur, at least in our approximations. The semi-classical expansion is completely stable against vacuum-like fluctuations and this stability is a highly robust property of the non-commutative gauge theory.

The quantum field theory is thus always dominated by the fluxons of non-commutative gauge theory which in the string representation correspond to unstable D-instantons in the two-dimensional target spacetime. In fact, the fluxon contributions are completely well defined for all values of the coupling $\mu=g^{2} \Theta$, as emphasized by figure 6 which illustrates that the fluxon free energy is generically a smooth function. Using properties of the Jonquière function, one can show that all derivatives of $\mathcal{F}_{\infty}\left(g^{2}, \Theta\right)$ with respect to $\mu$ vanish at $\mu=0$. The weak-coupling limit of the non-commutative gauge theory seems therefore completely regular and the expected singularity, representing a sort of Douglas-Kazakov phase transition at vanishing coupling, is resolved by non-commutativity. Again, this non-singular behaviour is a highly non-trivial feature of the non-commutative gauge theory.

At the other end of the parameter region, we can expand the fluxon contribution (4.39) in the strong-coupling limit $g^{2} \Theta \rightarrow \infty$, which is the regime 


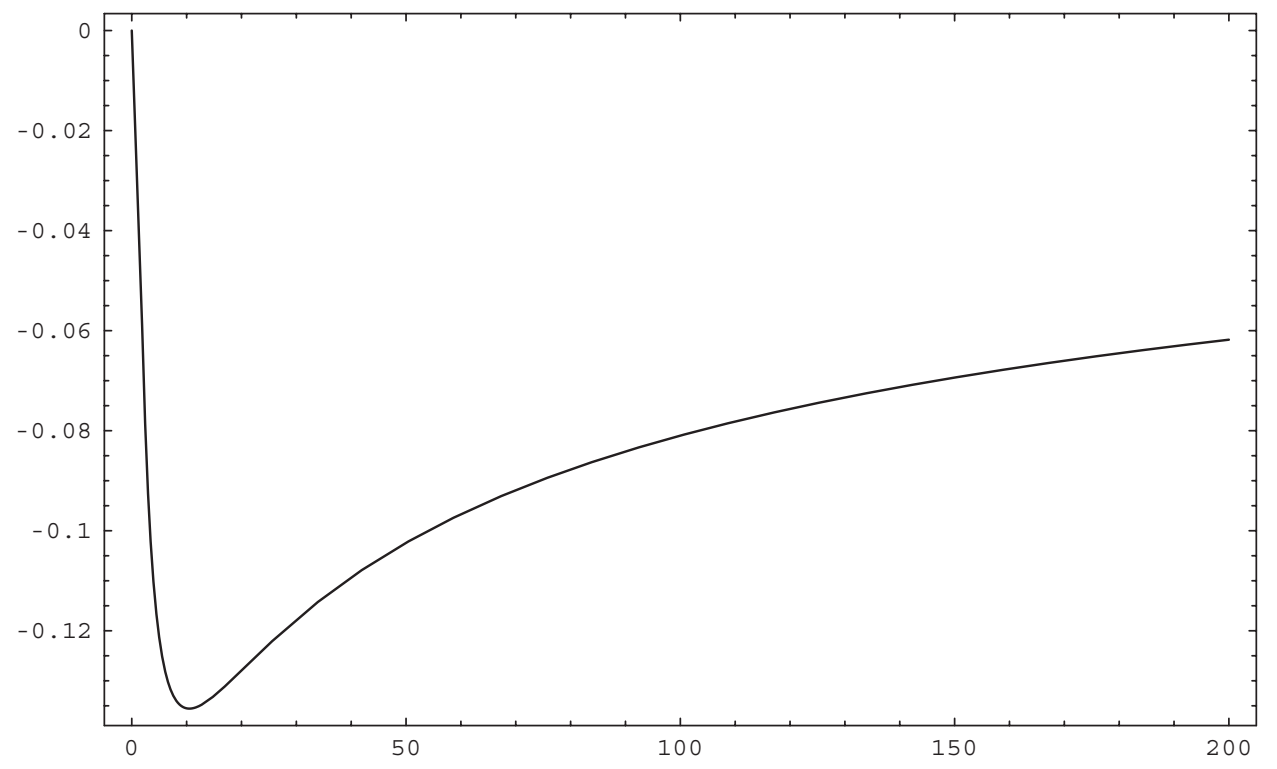

Figure 6: The fluxon free energy $\mathcal{F}_{\infty}$ as a function of the double-scaling parameter $\mu=g^{2} \Theta$.

in which we can compare to the commutative string expansion of the previous section. In fact, since the large $\Theta$ expansion of any non-commutative field theory is known to organize itself much like the large $N$ expansion of an ordinary multicolour field theory, this is precisely the limit where we would hope to be able to explicitly extract stringy behaviour of the noncommutative gauge theory. The free energy $\widehat{\mathcal{F}}_{\infty}\left(g^{2}, \Theta\right)=\ln \widehat{\mathcal{Z}}_{\infty}\left(g^{2}, \Theta\right)$ can be computed as an asymptotic series in $1 / \Theta$ by using the singular expansion of the Jonquière function $\mathrm{Li}_{3 / 2}(z)$ around its finite branch point at $z \rightarrow 1^{-}$ (see Appendix A, equation (A.5)), and one finds

$$
\begin{aligned}
& \lim _{\Theta \rightarrow \infty} \widehat{\mathcal{F}}_{\infty}\left(g^{2}, \Theta\right) \\
& =\frac{A}{2 \Theta}\left[\frac{g^{2} \Theta}{12}+\frac{2 \sqrt{\pi}}{g^{2} \Theta}-\frac{1}{\sqrt{\pi}} \sum_{k=0}^{\infty} \frac{(-1)^{k} \zeta\left(\frac{3}{2}-k\right)}{k !} \frac{1}{\left(g^{2} \Theta\right)^{k+1 / 2}}\right] .
\end{aligned}
$$

Non-perturbative corrections to this expansion (for finite $g^{2} \Theta<\infty$ ) are of order $\mathrm{e}^{-g^{2} \Theta}$. It is amusing to note that, starting from the gauge theory on $\mathbb{T}_{\theta}^{2}$ and assuming continuity in $\Theta$, the non-commutative gauge theory naively approaches the $\mathrm{SU}(N) / \mathbb{Z}_{N}$ gauge theory at large $N$ in the limit $\Theta, A \rightarrow \infty$ with $g \Theta / \sqrt{A}$ held fixed. This suggests another large $\Theta$ limit in which string behaviour could potentially be extracted from the gauge dynamics. 


\subsection{Open string interpretation}

In the strong-coupling phase analysed in the previous section, the physical degrees of freedom are dominated by a tree-level resummation of the D1-branes described in Section 6.4, of tension (6.42), which can be thought of as dual to the usual electric dipole quanta of non-commutative gauge theory. We will now reconcile this interpretation, and in particular the open string worldsheet perturbation expansion of Section 6.4, with the standard string interpretation of fluxons in non-commutative Yang-Mills theory. Consider a D-string extended along $\mathbb{R}^{2}$ and subjected to a constant, perpendicularly applied background NS-NS field $B$. The low-energy effective field theory of this system is described by non-commutative Yang-Mills theory with 16 supercharges and the open string parameters

$$
\Theta=\frac{1}{B}, \quad g^{2}=\frac{2 \pi \alpha^{\prime} g_{\mathrm{s}} B}{A} .
$$

This identification holds in the Seiberg-Witten decoupling limit $\left(\alpha^{\prime}\right)^{2} B \gg A$ wherein the string scale is small compared to the scale of non-commutativity [10]. Using it, we may identify the strong coupling expansion (7.18) with the string partition function

$$
\begin{aligned}
\mathcal{Z}_{\mathrm{str}}^{\infty}\left(B, T_{1} A\right) & :=\lim _{\Theta \rightarrow \infty} \widehat{\mathcal{F}}_{\infty}\left(g^{2}, \Theta\right) \\
& =\frac{A B}{2}\left[\frac{1}{6 T_{1} A}+\sqrt{\pi} T_{1} A-\sqrt{\frac{T_{1} A}{2 \pi}} \sum_{k=0}^{\infty} \frac{(-1)^{k} \zeta\left(\frac{3}{2}-k\right)}{2^{k} k !}\left(T_{1} A\right)^{k}\right] .
\end{aligned}
$$

Expansion (7.20) is to be compared with (6.44). We see that in the presence of the background $B$-field the open string vacuum amplitude becomes a non-analytic function of the worldsheet expansion parameter $T_{1} A$. The most striking distinction though is that, since $\zeta\left(\frac{3}{2}-k\right) \in \mathbb{R} \backslash \mathbb{Q} \forall k \in \mathbb{N}_{0}$, the coefficients in the expansion of the partition function are now irrational numbers. In this way, the $B$-field deforms the moduli spaces that arise in the double-scaling expansion of the $U(N)$ gauge theory of the previous section to ones with generically irrational normalized volumes. It is tempting to conjecture that, at least in the strong-coupling regime, these deformed moduli spaces, and the non-commutative open string theory, could be described by some sort of non-commutative topological sigma-model. Non-commutative versions of the standard topological field theories may be constructed by deforming the BRST algebras of observables [76-78].

Thus the D-string interpretation of ordinary two-dimensional Yang-Mills theory is consistent with that of the non-commutative case, and in the 
double-scaling limit it provides a toy laboratory to explore the stringy aspects of non-commutative gauge theory. In this language, the mass $E_{\ell}$ of a fluxon of charge $\ell \in \mathbb{Z}$ can be written as

$$
E_{\ell}=\frac{\pi|\ell|}{g^{2} \Theta}=\frac{\pi}{2}|\ell| T_{1} A
$$

This coincides with the Nambu-Goto action for $|\ell|$ D-strings, with $\ell$ the bound D-instanton charge. It is intriguing to now examine the lift of our two-dimensional constructions to $3+1$ dimensions, whereby the unstable fluxon solutions are known to correspond to full stable BPS solutions of $\mathcal{N}=4$ non-commutative gauge theory describing $|\ell|$ infinite D-strings piercing a D3-brane $[26,28,30]$. In the context of the present paper, they are obtained by embedding our non-commutative instantons on $\mathbb{T}_{\theta}^{2}$ in two of the three spatial dimensions of the low-energy effective field theory, and interpreting the resulting object as a string infinitely stretched in the remaining spatial dimension. After decompactification via the double-scaling limit, we obtain a configuration of static Euclidean strings on $\mathbb{R}^{2} \times \mathbb{R}_{\Theta}^{2}$. From (7.21), it follows that these instanton strings are equivalent to the D-strings that appear in the non-perturbative sector of the large $N$ expansion of ordinary Yang-Mills theory in the double-scaling limit. Given the S-duality between these string solitons and the string of $3+1$-dimensional non-commutative open string theory [79], it is natural to expect that the electric dipole degrees of freedom of the gauge theory on $\mathbb{T}_{\theta}^{2}$ map in an analogous way onto noncommutative open strings. This prospect is consistent with the S-duality, in $1+1$ dimensions, between non-commutative open string theory and ordinary Yang-Mills theory with non-zero electric flux [80].

\section{Acknowledgments}

We thank L. Paniak for early collaboration on the issues described in Section 3. We are grateful to A. Bassetto, G. Cicuta, F. Colomo, Ph. Flajolet, D. Johnston, M. Matone, A. Polychronakos, S. Ramgoolam and J. Wheater for helpful discussions and correspondence. R.J.S. would like to thank the Department of Physics at the University of Parma and the Department of Physics at the University of Florence for their hospitality during various stages of this work. The work of R.J.S. was supported in part by an Advanced Fellowship from the Particle Physics and Astronomy Research Council (U.K.). 


\section{A. Properties of the Jonquière function}

In this appendix we will describe some elementary properties of the polylogarithm function

$$
\operatorname{Li}_{\alpha}(z)=\sum_{k=1}^{\infty} \frac{z^{k}}{k^{\alpha}}
$$

for $z, \alpha \in \mathbb{C}$. It can be regarded as a generalization of the Riemann zetafunction which is obtained in the limit $z=1, \operatorname{Li}_{s}(1)=\zeta(s)$. In particular, we will derive a new asymptotic expansion formula for it, which is the crux of the saddle-point analyses of the main text. Some related results are found in [81].

\section{A.1 Analytic continuation}

The series (A.1) is absolutely convergent everywhere on the open unit disk $|z|<1$. To extend it analytically into the entire complex plane $z \in \mathbb{C}$, we use a Mellin transform to write the integral representation

$$
\operatorname{Li}_{\alpha}(z)=\sum_{k=1}^{\infty} \frac{1}{\Gamma(\alpha)} \int_{0}^{\infty} \mathrm{d} t t^{\alpha-1} \mathrm{e}^{-t k} z^{k}=\frac{z}{\Gamma(\alpha)} \int_{0}^{\infty} \mathrm{d} t \frac{t^{\alpha-1}}{\mathrm{e}^{t}-z}
$$

which is valid when $\operatorname{Re}(\alpha)>0$. By using the derivative identity

$$
\operatorname{Li}_{\alpha}(z)=z \frac{\mathrm{d}}{\mathrm{d} z} \operatorname{Li}_{\alpha+1}(z)
$$

along with the integral representation (A.2) for the function $\operatorname{Li}_{\alpha+1}(z)$, the change of variables $s=\mathrm{e}^{t}$ leads to the alternative form

$$
\operatorname{Li}_{\alpha}(z)=\frac{z}{\Gamma(\alpha+1)} \int_{1}^{\infty} \mathrm{d} s \frac{\ln ^{\alpha} s}{(s-z)^{2}}
$$

valid when $\operatorname{Re}(\alpha)>-1$. This expression exhibits a branch cut singularity of $\operatorname{Li}_{\alpha}(z)$ along $z \in[1, \infty)$. Its behaviour near the branch point at $z=1$ is given by the series expansion [81]

$$
\operatorname{Li}_{\alpha}(z)=\Gamma(1-\alpha)(-\ln z)^{\alpha-1}+\sum_{k=0}^{\infty} \frac{\zeta(\alpha-k)}{k !} \ln ^{k} z, \quad z \rightarrow 1^{-} .
$$

\section{A.2 Asymptotic expansion}

We are now ready to derive our new asymptotic expansion of the Jonquière function, which we have also checked numerically using Mathematica. 
Theorem 3. The asymptotic expansion as $x \rightarrow \infty$ of the polylogarithm function $\mathrm{Li}_{\alpha}\left(-\mathrm{e}^{x}\right)$ is given by

$$
\operatorname{Li}_{\alpha}\left(-\mathrm{e}^{x}\right)=-2 \sum_{k=0}^{\infty} \frac{\left(1-2^{1-2 k}\right) \zeta(2 k)}{\Gamma(\alpha+1-2 k)} x^{\alpha-2 k}+\mathrm{O}\left(\mathrm{e}^{-x}\right) .
$$

Proof. For $x \in[0, \infty)$, we start from the integral representation (A.4) and change integration variables as $y=\mathrm{e}^{-x} s$ to write

$$
\operatorname{Li}_{\alpha}\left(-\mathrm{e}^{x}\right)=-\frac{1}{\Gamma(\alpha+1)} \int_{\mathrm{e}^{-x}}^{\infty} \mathrm{d} y \frac{(\ln y+x)^{\alpha}}{(y+1)^{2}} .
$$

Let us decompose the domain of integration in (A.6) as the disjoint union $\left[\mathrm{e}^{-x}, \mathrm{e}^{x}\right) \cup\left[\mathrm{e}^{x}, \infty\right)$. It is straightforward to show that the contribution to the integral (A.6) from the second component is exponentially suppressed. For this, we use the fact that $y \in\left[\mathrm{e}^{x}, \infty\right)$ is large for $x \rightarrow \infty$ to expand

$$
\begin{aligned}
\int_{\mathrm{e}^{x}}^{\infty} \mathrm{d} y \frac{(\ln y+x)^{\alpha}}{(y+1)^{2}} & =-\sum_{n=1}^{\infty}(-1)^{n} n \int_{\mathrm{e}^{x}}^{\infty} \frac{\mathrm{d} y}{y^{n+1}}(\ln y+x)^{\alpha} \\
& =-\sum_{n=1}^{\infty}(-1)^{n} n \mathrm{e}^{n x} \int_{2 x}^{\infty} \mathrm{d} u u^{\alpha} \mathrm{e}^{-n u} \\
& =-\sum_{n=1}^{\infty} \frac{(-1)^{n}}{n^{\alpha}} \mathrm{e}^{n x} \Gamma(\alpha+1,2 n x) .
\end{aligned}
$$

Let us now recall the large $x$ behaviour of the incomplete Euler gammafunction given by [82]

$$
\Gamma(\nu, z)=z^{\nu-1} \mathrm{e}^{-z}\left[\sum_{m=0}^{l-1} \frac{(-1)^{m}}{z^{m}} \frac{\Gamma(1-\nu+m)}{\Gamma(1-\nu)}+O\left(|z|^{-l}\right)\right]
$$

with $|z| \rightarrow \infty,-\frac{3 \pi}{2}<\arg z<\frac{3 \pi}{2}$, and $l \in \mathbb{N}$. By keeping only the lowest order $l=1$ term in (A.8), one finds that the integral (A.7) is given approximately for large $x$ as

$$
\int_{\mathrm{e}^{x}}^{\infty} \mathrm{d} y \frac{(\ln y+x)^{\alpha}}{(y+1)^{2}} \simeq 2^{\alpha} x^{\alpha} \mathrm{e}^{-x},
$$

exhibiting the claimed exponential suppression at $x \rightarrow \infty$. 
It remains to compute the integral (A.6) over the first component. For $y \in\left[\mathrm{e}^{-x}, \mathrm{e}^{x}\right)$ one has $|\ln (y) / x| \leq 1$, and so we may expand

$$
\int_{\mathrm{e}^{-x}}^{\mathrm{e}^{x}} \mathrm{~d} y \frac{(\ln y+x)^{\alpha}}{(y+1)^{2}}=\sum_{n=0}^{\infty} \frac{\Gamma(\alpha+1)}{\Gamma(n+1) \Gamma(\alpha+1-n)} x^{\alpha-n} \int_{\mathrm{e}^{-x}}^{\mathrm{e}^{x}} \mathrm{~d} y \frac{\ln ^{n} y}{(y+1)^{2}} .
$$

By performing the change of variable $y \rightarrow 1 / y$, one can deduce that the integrals in (A.10) vanish for odd $n$. For $n=2 k$ even, the integrals can be computed by means of the change of integration variable $y=\mathrm{e}^{v}$, and neglecting exponentially suppressed terms in the limit $x \rightarrow \infty$ one finds after integrating by parts the result

$$
\begin{aligned}
\int_{\mathrm{e}^{-x}}^{\mathrm{e}^{x}} \mathrm{~d} y \frac{\ln ^{2 k} y}{(y+1)^{2}} & =4 k \int_{0}^{\infty} \mathrm{d} v \frac{v^{2 k+1}}{\mathrm{e}^{v}+1}+\mathrm{O}\left(\mathrm{e}^{-x}\right) \\
& =2\left(1-2^{1-2 k}\right) \Gamma(2 k+1) \zeta(2 k)+\mathrm{O}\left(\mathrm{e}^{-x}\right) .
\end{aligned}
$$

By substituting this into (A.10), we arrive finally at the claimed asymptotic expansion of the polylogarithm function (A.6).

This formula can be expressed in terms of Bernoulli numbers by using the Euler identity [82]

$$
\zeta(2 k)=\frac{2^{2 k-1} B_{2 k} \pi^{2 k}}{(2 k) !}, \quad k \in \mathbb{N}_{0}
$$

\section{B. Free fermion representation}

Proposition 1 combined with Theorem 2 determines the asymptotic behaviour as $n \rightarrow \infty$ of the combinatorial formula (5.7) and (5.10) via the strongcoupling expansion of the gauge theory in the double-scaling limit. The explicit calculation was performed by solving a saddle-point equation that avoids the very difficult task of obtaining these asymptotic combinatorics directly. In this section, we sketch an alternative derivation based on the standard free fermion representation of two-dimensional Yang-Mills theory $[51,52]$, which gives a useful fermionic picture of the double-scaling limit whose potential extension to the case of non-trivial fluxes could provide a free fermion formulation of non-commutative gauge theory. Moreover, it immediately yields another proof of the quasi-modularity of the free energy as described in Section 5.4. The idea is to consider a more general amplitude which contains the pertinent information about the chiral partition function [61], but which has a much simpler and tractable behaviour in the double-scaling limit. 
Let us introduce free chiral fermion fields

$$
\psi(z)=\sum_{r \in \mathbb{Z}+\frac{1}{2}} \psi_{r} z^{-r} \sqrt{\frac{\mathrm{d} z}{2 \pi \mathrm{i} z}}, \quad \psi^{*}(z)=\sum_{r \in \mathbb{Z}+\frac{1}{2}} \psi_{r}^{*} z^{-r} \sqrt{\frac{\mathrm{d} z}{2 \pi \mathrm{i} z}}
$$

for $z \in \mathbb{C}$ obeying the non-vanishing canonical anticommutation relations

$$
\left\{\psi_{r}, \psi_{s}^{*}\right\}=\delta_{r+s, 0}
$$

They act on the standard fermionic Fock space defined by

$$
\mathcal{F}_{\mathrm{F}}=\bigoplus_{k, l=1}^{\infty} \bigoplus_{\substack{r \in \mathbb{N}^{k} \\ r_{1}<r_{2}<\cdots<r_{k}}} \bigoplus_{\substack{\boldsymbol{s} \in \mathbb{N}^{l} \\ s_{1}<s_{2}<\cdots<s_{l}}} \mathbb{C} \cdot \prod_{i=1}^{k} \psi_{-r_{i}} \prod_{j=1}^{l} \psi_{-s_{j}}^{*}|0\rangle,
$$

where $|0\rangle$ is the Fermi vacuum defined by the annihilation conditions

$$
\psi_{r}|0\rangle=\psi_{r}^{*}|0\rangle=0 \quad \forall r>0 .
$$

Using the fermion fields (B.1), we define the $U(1)$ charge operator

$$
J_{0}:=\oint_{\mathcal{C}_{0}} \circ \psi(z) \psi^{*}(z) \stackrel{\circ}{\circ}=\sum_{r \in \mathbb{Z}+\frac{1}{2}} \circ \psi_{r} \psi_{-r}^{*} \circ,
$$

where $\stackrel{\circ}{\circ} \cdot \circ$ denotes normal ordering with respect to the vacuum $|0\rangle$. The Fock space (B.3) then admits a natural $\mathbb{Z}$-grading by total $U(1)$ charge as $\mathcal{F}_{\mathrm{F}}=\bigoplus_{q \in \mathbb{Z}} \mathcal{F}_{\mathrm{F}}^{q}$, where $\mathcal{F}_{\mathrm{F}}^{q}$ is the eigenspace of the zero mode operator (B.5) with eigenvalue $q \in \mathbb{Z}$. A set of spanning vectors for this subspace is provided by the "partition basis" with

$$
\mathcal{F}_{\mathrm{F}}^{q}=\bigoplus_{n=0}^{\infty} \bigoplus_{\substack{n \in \mathbb{N}_{0}^{n}, \sum_{l} n_{l}=n \\ n_{1} \geq n_{2} \geq \cdots \geq n_{n}}} \mathbb{C} \cdot \prod_{l=1}^{n} \psi_{-q-\frac{1}{2}+l-n_{l}}|q-n\rangle
$$

where $|q\rangle$ is the Fermi vacuum of overall $U(1)$ charge $q$, i.e., $J_{0}|q\rangle=q|q\rangle$, which is defined by

$$
\psi_{r}|q\rangle=\psi_{s}^{*}|q\rangle=0 \quad \forall r>q, \quad s>-q .
$$

Let us now introduce the free fermion Hamiltonian operator

$$
L_{0}:=\oint_{\mathcal{C}_{0}}{ }_{\circ}^{\circ} \psi(z) z \frac{\mathrm{d}}{\mathrm{d} z} \psi^{*}(z)_{\circ}^{\circ}=\sum_{r \in \mathbb{Z}+\frac{1}{2}} r \stackrel{\circ}{\circ} \psi_{r} \psi_{-r}^{*} \circ
$$

with $L_{0}|q\rangle=\left(q^{2} / 2\right)|q\rangle$. We are interested in the dynamics generated by this Hamiltonian in a subspace $\mathcal{F}_{\mathrm{F}}^{q}$ of fixed $U(1)$ charge. According to (B.6), it is in such subspaces that we can make contact with the Yang-Mills vacuum amplitude (5.4) through the representation of the sum over Young diagrams in terms of partitions. For simplicity, we will work in the eigenspace of charge $q=0$, i.e., $\mathcal{F}_{\mathrm{F}}^{0}=\operatorname{ker} J_{0}$. This essentially constrains us to representations of 
$\mathrm{SU}(N)$ and it describes the low-energy excitations (compared to $N$ ) of the fermion system around the Fermi level (5.28).

We will study the correlation functions of the scaled charge operators

$$
J_{0}(w):=\oint_{\mathcal{C}_{0}} \circ{ }_{\circ}^{\circ} \psi\left(w^{-1 / 2} z\right) \psi^{*}\left(w^{1 / 2} z\right) \circ=\sum_{r \in \mathbb{Z}+\frac{1}{2} \mid}{ }_{\circ}^{\circ} \psi_{r} \psi_{-r}^{*} \circ{ }_{\circ}^{\circ} w^{r},
$$

with $J_{0}(1)=J_{0}$, for $w \in \mathbb{C}$ in the subspace ker $J_{0}$. Consider the amplitude, defined for every non-negative integer $m$, given as a trace over states of vanishing $U(1)$ charge by

$$
\left\langle\prod_{i=1}^{m} J_{0}\left(w_{i}\right)\right\rangle_{0}:=\operatorname{Tr}_{\operatorname{ker} J_{0}}\left(\mathrm{e}^{-\lambda L_{0}} \prod_{i=1}^{m} J_{0}\left(w_{i}\right)\right) .
$$

By using the partition basis (B.6) at $q=0$, this correlator may be written explicitly as

$$
\left\langle\prod_{i=1}^{m} J_{0}\left(w_{i}\right)\right\rangle_{0}=\sum_{n=1}^{\infty} \mathrm{e}^{-n \lambda} \sum_{\substack{n \in \mathbb{N}_{0}^{n}, \sum_{l} n_{l}=n \\ n_{1} \geq n_{2} \geq \cdots \geq n_{n}}} \prod_{k=1}^{m}\left(\sum_{i=1}^{n} w_{k}^{n_{i}-i+1 / 2}\right) .
$$

The correlation function (B.11) admits a nice physical interpretation. Let us write the complex coordinates in the parametrization $z=\mathrm{e}^{t+\mathrm{i} \sigma}$, where $(t, \sigma) \in \mathbb{R} \times \mathbb{S}^{1}$ are coordinates on the cylinder in radial quantization of the two-dimensional fermion system. The partitions $\boldsymbol{n} \in \mathbb{N}_{0}^{n}$ occuring in the second sum in (B.6), with $\sum_{l} n_{l}:=\sum_{j} j \nu_{j}=n$, are in one-to-one correspondence with (unramified) $n$-sheeted oriented coverings of the spatial circle $\mathbb{S}^{1}[4,5]$. Each basis state in (B.6) may then be identified with a state in the Fock space of closed strings defined by $\nu_{j}$ strings with winding number $j$, which we may interpret as electric flux around the circle. The worldsheet swept out by each such string in periodic time defines a $j$-fold cover of a torus by a torus. Since the Hamiltonian (B.8) is not diagonal in the partition basis, the interactions of strings create branch cuts on the base torus $\mathbb{T}^{2}$ and glue copies of it into higher genus Riemann surfaces. The correlator (B.11) is the generating function for the (topological classes of) covers of $\mathbb{T}^{2}$ constructed from $m$ branch points, i.e., for arbitrary (not necessarily simple) Hurwitz numbers, with $w_{k}$ weighting the branch points of various orders. The branch points themselves correspond to $U(1)$ charge insertions at $w_{k}$ of the fermions defined by the field (B.9). The $k$ th Laurent coefficient of (B.11) near the point $w_{1}=\cdots=w_{m}=1$ determines the number of covers with branch points of order $k$. Extracting this coefficient is tantamount to integrating over the positions of the corresponding branch points. 
In the case of chiral Yang-Mills theory on $\mathbb{T}^{2}$, the subspace $\mathcal{F}_{\mathrm{F}}^{0}$ may be identified with the space of "representation states" contributing to the partition function (5.6). In this way, the double-scaling limit precisely factors out the chiral sector from the weakly coupled chiral-anti-chiral string expansion of two-dimensional Yang-Mills theory. In light of this discussion, the genus $h$ contribution $Z_{h}^{+}(\lambda)$ to the Yang-Mills partition function (5.6) may be extracted from the second Laurent coefficient of (B.11) with $m=2 h-2$ (corresponding to the case of covers of $\mathbb{T}^{2}$ with only simple ramification). For this, we associate to each non-increasing partition $\boldsymbol{n} \in \mathbb{N}_{0}^{n}$ occuring in the second sum in (B.11) a Young diagram $Y \in \mathcal{Y}_{n}$ and corresponding $U(N)$ representation $R(Y)$ as described in Section 5.1, and evaluate the central character $\widetilde{C}_{2}(\boldsymbol{n}):=\widetilde{C}_{2}(R(Y))$ as in $(5.5)$ to compute

$$
Z_{h}^{+}(\lambda)=\lambda^{2 h-2} \sum_{n=1}^{\infty} \mathrm{e}^{-n \lambda} \sum_{\substack{\boldsymbol{n} \in \mathbb{N}_{0}^{n}, \sum_{l} n_{l}=n \\ n_{1} \geq n_{2} \geq \cdots \geq n_{n}}} \widetilde{C}_{2}(\boldsymbol{n})^{2 h-2} .
$$

By using the identity

$$
\widetilde{C}_{2}(\boldsymbol{n})=\left.\left(w \frac{\mathrm{d}}{\mathrm{d} w}\right)^{2}\right|_{w=1} \sum_{i=1}^{n} w^{n_{i}-i+1 / 2 \mid}-\frac{n\left(4 n^{2}-1\right)}{12},
$$

we may then write the partition function (B.12) in terms of the correlator (B.11) as

$$
Z_{h}^{+}(\lambda)=\left.\lambda^{2 h-2} \prod_{i=1}^{2 h-2}\left(w_{i} \frac{\mathrm{d}}{\mathrm{d} w_{i}}\right)^{2}\right|_{w_{i}=1}\left\langle\prod_{j=1}^{2 h-2} J_{0}\left(w_{j}\right)\right\rangle_{0}
$$

Once (B.14) is determined, we may use the following combinatorial trick to compute the genus $h$ free energy (5.9) [61]. Let $\boldsymbol{\Pi}_{h}^{+}$be the set of all partitions of the set $\{1, \ldots, 2 h-2\}$ into unordered disjoint unions of nonempty subsets of even cardinality. Given $\boldsymbol{\sigma}:=\coprod_{k=1}^{L} \sigma_{k} \in \boldsymbol{\Pi}_{h}^{+}$, the number $L=L(\boldsymbol{\sigma})$ is the length of the partition $\boldsymbol{\sigma}$. By definition, since $Z_{h}^{+}(\lambda)$ is the generating function of all genus $h$ simple covers of $\mathbb{T}^{2}$, and $F_{h}^{+}(\lambda)$ that of the connected coverings, we have the identity

$$
Z_{h}^{+}(\lambda)=Z_{1}^{+}(\lambda) \sum_{\boldsymbol{\sigma} \in \boldsymbol{\Pi}_{h}^{+}} \prod_{k=1}^{L(\boldsymbol{\sigma})} F_{\left|\sigma_{k}\right|}^{+}(\lambda)
$$

where $Z_{1}^{+}(\tau)=\mathrm{e}^{-\pi \mathrm{i} \tau / 12} \eta(\tau)$ is the generating function for coverings without unramified connected components. Applying a standard Möbius inversion to (B.15) [83], we may then determine the free energy contributions 
through the combinatorial formula

$$
F_{h}^{+}(\lambda)=\sum_{\boldsymbol{\sigma} \in \boldsymbol{\Pi}_{h}^{+}}(-1)^{L(\boldsymbol{\sigma})}(L(\boldsymbol{\sigma})-1) ! \prod_{k=1}^{L(\boldsymbol{\sigma})} \frac{Z_{\left|\sigma_{k}\right|}^{+}(\lambda)}{Z_{1}^{+}(\lambda)} .
$$

The main advantage of this rewriting is that the amplitude (B.11) can be computed explicitly. It defines a meromorphic function which is absolutely convergent in the domain of the complex plane defined by the conditions $\mathrm{e}^{-\lambda}<\left|w_{i_{1}} \cdots w_{i_{k}}\right|<\mathrm{e}^{\lambda}$ for any subset $\left\{i_{1}, \ldots, i_{k}\right\} \subset\{1, \ldots, m\}$. Its only singularities are simple poles along the divisors $w_{i}=1, i=1, \ldots, m$. This uniquely determines the correlation functions up to an additive constant in terms of the single odd characteristic elliptic Jacobi theta-function

$$
\vartheta(w, \tau):=\sum_{n=-\infty}^{\infty}(-1)^{n} w^{n+1 / 2} \mathrm{e}^{\pi \mathrm{i}(n+1 / 2)^{2} \tau},
$$

and its derivatives

$$
\vartheta^{(k)}(w, \tau):=\left(w \frac{\mathrm{d}}{\mathrm{d} w}\right)^{k} \vartheta(w, \tau)
$$

as $[84]$

$$
\left\langle\prod_{i=1}^{m} J_{0}\left(w_{i}\right)\right\rangle_{0}=\sum_{\pi \in S_{m}} \frac{\operatorname{det}\left[\vartheta^{(j-i+1)}\left(w_{\pi(1)} \cdots w_{\pi(m-j)}, \tau\right) /(j-i+1) !\right]_{1 \leq i, j \leq m}}{\vartheta\left(w_{\pi(1)}, \tau\right) \vartheta\left(w_{\pi(1)} w_{\pi(2)}, \tau\right) \cdots \vartheta\left(w_{\pi(1)} \cdots w_{\pi(m)}, \tau\right)}
$$

with $1 /(-k) !:=0 \forall k \geq 1$. The free energy (B.16) is then computed from (B.19) using (B.14) along with the identity [84]

$$
\vartheta^{(2 m+1)}(1, \tau)=(2 m+1) ! \sum_{\substack{\boldsymbol{\nu} \in \mathbb{N}_{0}^{m} \\ \sum_{l} l \nu_{l}=m}} \prod_{k=1}^{m} \frac{1}{\nu_{k} !}\left[\frac{B_{2 k} E_{2 k}(\tau)}{k(2 k) !}\right]^{\nu_{k}},
$$

and $\vartheta^{(2 m)}(1, \tau)=0 \forall m \geq 1$. These formulas demonstrate explicitly the quasi-modularity of the free energy that we described in Section 5.1 [53, $61,84]$, and moreover give, at least in principle, the explicit expansion of the free energy into the Eisenstein series basis for the ring $\mathcal{M}$ of quasi-modular forms.

In the present context, the principal characteristic of the formula (B.19) is that its form in the double-scaling limit can be made very explicit. By using the Poisson resummation formula (5.26), one may derive the weak-coupling 
behaviour of the Jacobi theta-function (B.17) to be

$$
\frac{\vartheta(w, \tau)}{\vartheta^{(1)}(1, \tau)}=\frac{\lambda}{\pi} \sin \left(\frac{\pi w}{\lambda}\right) \mathrm{e}^{w^{2} / 2 \lambda}+\mathrm{O}\left(\mathrm{e}^{-1 / \lambda}\right)
$$

and use the generating function

$$
\frac{\pi w}{\sin \pi w}=2 \sum_{k=0}^{\infty}\left(1-2^{1-2 k}\right) \zeta(2 k) w^{2 k} .
$$

One can now proceed to derive the asymptotics of the formula (B.19), and substitute into (B.14) and (B.16) to compute finally the double-scaling free energy at genus $h$. We will not enter into the details of this tedious combinatorial calculation, but refer to [61] for the technical details. The final result agrees with Theorem 2.

More generally, the two limits of two-dimensional Yang-Mills theory that we have considered in this paper seem to have natural realizations in the fermionic picture. The standard 't Hooft limit is the one in which the length of the (dual) circle where the fermions move is kept finite, while their number goes to infinity. The low-lying spectrum becomes universal and equidistant (with $1 / N$ corrections) and this is the conformal field theory limit described by a $c=1$ theory $[51,52]$. The spatial density of fermions, however, goes to infinity. On the other hand, the double-scaling limit appears to be more akin to the thermodynamic limit of the free fermion system [85-87], in which the size of the circle scales like $N$ leading to an infinite volume system with a finite particle density. In this limit, the spectrum becomes continuous and an infinity of states must be taken into account to calculate correlation functions. It would be interesting to make this connection more precise, and to extend it to a fermionic formulation of non-commutative gauge theory in two dimensions.

\section{References}

[1] G. 't Hooft, A planar diagram theory for strong interactions, Nucl. Phys. B 72 (1974), 461.

[2] J. Maldacena, The large $N$ limit of superconformal field theories and supergravity, Adv. Theor. Math. Phys. 2 (1998), 231 [hep-th/9711200].

[3] D.J. Gross, Two-dimensional QCD as a string theory, Nucl. Phys. B 400 (1993), 161 [hep-th/9212149].

[4] D.J. Gross and W. Taylor, Two-dimensional QCD is a string theory, Nucl. Phys. B 400 (1993), 181 [hep-th/9301068]. 
[5] D.J. Gross and W. Taylor, Twists and Wilson loops in the string theory of two-dimensional QCD, Nucl. Phys. B 403 (1993), 395 [hep-th/9303046].

[6] R. Gopakumar and C. Vafa, On the gauge theory/geometry correspondence, Adv. Theor. Math. Phys. 3 (1999), 1415 [hep-th/9811131].

[7] A. Konechny and A. Schwarz, Introduction to matrix theory and noncommutative geometry, Phys. Rept. 360 (2002), 353 [hep-th/0012145]; [hep-th/0107251].

[8] M.R. Douglas and N.A. Nekrasov, Non-commutative field theory, Rev. Mod. Phys. 73 (2002), 977 [hep-th/0106048].

[9] R.J. Szabo, Quantum field theory on non-commutative spaces, Phys. Rept. 378 (2003), 207 [hep-th/0109162].

[10] N. Seiberg and E. Witten, String theory and non-commutative geometry, JHEP 9909 (1999), 32 [hep-th/9908142].

[11] F. Lizzi, R.J. Szabo and A. Zampini, Geometry of the gauge algebra in non-commutative Yang-Mills theory, JHEP 108 (2001), 32 [hep-th/0107115].

[12] L.D. Paniak and R.J. Szabo, Instanton expansion of non-commutative gauge theory in two dimensions, Commun. Math. Phys. 243 (2003), 343 [hep-th/0203166].

[13] L.D. Paniak and R.J. Szabo, Open Wilson lines and group theory of non-commutative Yang-Mills theory in two dimensions, JHEP 305 (2003), 29 [hep-th/0302162].

[14] L.D. Paniak and R.J. Szabo, Lectures on two-dimensional noncommutative gauge theory 2: quantization, hep-th/0304268.

[15] L. Griguolo and D. Seminara, Classical solutions of the TEK model and non-commutative instantons in two dimensions, JHEP 403 (2004), 68 [hep-th/0311041].

[16] J. Ambjørn, Y.M. Makeenko, J. Nishimura and R.J. Szabo, Finite N matrix models of non-commutative gauge theory, JHEP 9911 (1999), 29 [hep-th/9911041]; Nonperturbative dynamics of non-commutative gauge theory, Phys. Lett. B 480 (2000), 399 [hep-th/0002158]; Lattice gauge fields and discrete non-commutative Yang-Mills theory, JHEP 0005 (2000), 23 [hep-th/0004147].

[17] T. Eguchi and H. Kawai, Reduction of dynamical degrees of freedom in the large $N$ gauge theory, Phys. Rev. Lett. 48 (1982), 1063.

[18] A. Gonzalez-Arroyo and M. Okawa, A twisted model for large $N$ lattice gauge theory, Phys. Lett. B 120 (1983), 174.

[19] T. Eguchi and R. Nakayama, Simplification of quenching procedure for large $N$ spin models, Phys. Lett. B 122 (1983), 59. 
[20] H. Steinacker, Quantized gauge theory on the fuzzy sphere as random matrix model, Nucl. Phys. B 679 (2004), 66 [hep-th/0307075].

[21] A. Hashimoto and N. Itzhaki, On the hierarchy between noncommutative and ordinary supersymmetric Yang-Mills, JHEP 9912 (1999), 007 [hep-th/9911057].

[22] S. Elitzur, B. Pioline and E. Rabinovici, On the short distance structure of irrational non-commutative gauge theories, JHEP 0010 (2000), 011 [hep-th/0009009].

[23] L. Alvarez-Gaumé and J.L.F. Barbón, Morita duality and large $N$ limits, Nucl. Phys. B 623 (2002), 165 [hep-th/0109176].

[24] G. Landi, F. Lizzi and R.J. Szabo, From large $N$ matrices to the non-commutative torus, Commun. Math. Phys. 217 (2001), 181 [hep-th/9912130].

[25] L. Griguolo, D. Seminara and P. Valtancoli, Towards the solution of non-commutative $\mathrm{YM}_{2}$ : Morita equivalence and large $N$ limit, JHEP 0112 (2001), 24 [hep-th/0110293].

[26] D.J. Gross and N.A. Nekrasov, Monopoles and strings in noncommutative gauge theory, JHEP 7 (2000), 34 [hep-th/0005204].

[27] A.P. Polychronakos, Flux tube solutions in non-commutative gauge theories, Phys. Lett. B 495 (2000), 407 [hep-th/0007043].

[28] D.J. Gross and N.A. Nekrasov, Dynamics of strings in non-commutative gauge theory, JHEP 10 (2000), 21 [hep-th/0007204].

[29] M. Aganagic, R. Gopakumar, S. Minwalla and A. Strominger, Unstable solitons in non-commutative gauge theory, JHEP 104 (2001), 1 [hep-th/0009142].

[30] D.J. Gross and N.A. Nekrasov, Solitons in non-commutative gauge theory, JHEP 103 (2001), 44 [hep-th/0010090].

[31] L. Griguolo, D. Seminara and R.J. Szabo, Two-dimensional Yang-Mills theory and moduli spaces of holomorphic differentials, Phys. Lett. B 600 (2004), 275 [hep-th/0408055]; Double scaling string theory of QCD in two dimensions, hep-th/0409288.

[32] T.M. Apostol, Modular functions and dirichlet series in number theory, Springer, 1976.

[33] M.R. Douglas, Large $N$ gauge theory: expansions and transitions, Nucl. Phys. Proc. Suppl. 41 (1995), 66 [hep-th/9409098].

[34] M.R. Douglas and V.A. Kazakov, Large $N$ phase transition in continuum QCD in two dimensions, Phys. Lett. B 319 (1993), 219 [hep-th/9305047]. 
[35] J.A. Minahan and A.P. Polychronakos, Classical solutions for twodimensional QCD on the sphere, Nucl. Phys. B 422 (1994), 172 [hep-th/9309119].

[36] D.J. Gross and A. Matytsin, Instanton induced large $N$ phase transitions in two-dimensional and four-dimensional QCD, Nucl. Phys. B 429 (1994), 50 [hep-th/9404004].

[37] G.H. Hardy and E.M. Wright, An introduction to the theory of numbers, Oxford, 1954.

[38] C.S. Chan, A. Hashimoto and H. Verlinde, Duality cascade and oblique phases in non-commutative open string theory, JHEP 109 (2001), 34 [hep-th/0107215].

[39] E. Langmann, R.J. Szabo and K. Zarembo, Exact solution of quantum field theory on non-commutative phase spaces, JHEP 401 (2004), 17 [hep-th/0308043].

[40] A.A. Migdal, Recursion equations in gauge field theories, Sov. Phys. JETP 42 (1975), 413.

[41] B.E. Rusakov, Loop averages and partition functions in $U(N)$ gauge theory on two-dimensional manifolds, Mod. Phys. Lett. A 5 (1990), 693.

[42] G. 't Hooft, Topology of the gauge condition and new confinement phases in nonabelian gauge theories, Nucl. Phys. B 190 (1981), 455.

[43] E.J. Martinec and G.W. Moore, Non-commutative solitons on orbifolds, hep-th/0101199.

[44] T. Krajewski and M. Schnabl, Exact solitons on non-commutative tori, JHEP 108 (2001), 2 [hep-th/0104090].

[45] G. Landi, F. Lizzi and R.J. Szabo, Matrix quantum mechanics and soliton regularization of non-commutative field theory, Adv. Theor. Math. Phys. 8 (2004), 1 [hep-th/0401072].

[46] A.D. Mednykh, Branched coverings of Riemann surfaces whose branch orders coincide with the multiplicity, Commun. Alg. 18 (1990), 1517.

[47] S. Monni, J.S. Song and Y.S. Song, The Hurwitz enumeration problem of branched covers and Hodge integrals, J. Geom. Phys. 50 (2004), 223 [hep-th/0009129].

[48] R.E. Rudd, The string partition function for $Q C D$ on the torus, hep-th/9407176.

[49] R. Dijkgraaf, Mirror symmetry and elliptic curves, Progr. Math. 129 (1995), 149; Chiral deformations of conformal field theories, Nucl. Phys. B 493 (1997), 588 [hep-th/9609022]. 
[50] M. Kaneko and D. Zagier, A generalized Jacobi theta function and quasi-modular forms, Progr. Math. 129 (1995), 165.

[51] M.R. Douglas, Conformal field theory techniques in large $N$ YangMills theory, NATO Adv. Sci. Inst. Phys. B 328 (1995), 119 [hep-th/9311130].

[52] J.A. Minahan and A.P. Polychronakos, Equivalence of two-dimensional $Q C D$ and the $c=1$ matrix model, Phys. Lett. B 312 (1993), 155 [hep-th/9303153].

[53] H. Ochiai, Counting functions for branched covers of elliptic curves and quasi-modular forms, math-ph/9909023.

[54] M. Bershadsky, S. Cecotti, H. Ooguri and C. Vafa, Holomorphic anomalies in topological field theories, Nucl. Phys. B 405 (1993), 279 [hep-th/9302103]; Kodaira-Spencer theory of gravity and exact results for quantum string amplitudes, Commun. Math. Phys. 165 (1994), 311 [hep-th/9309140].

[55] S. Cordes, G.W. Moore and S. Ramgoolam, Large N 2D Yang-Mills theory and topological string theory, Commun. Math. Phys. 185 (1997), 543 [hep-th/9402107].

[56] L. Griguolo, The instanton contributions to Yang-Mills theory on the torus: localization, Wilson loops and the perturbative expansion, Nucl. Phys. B 547 (1999), 375 [hep-th/9811050].

[57] M. Caselle, A. D'Adda, L. Magnea and S. Panzeri, Two-dimensional $Q C D$ is a one-dimensional Kazakov-Migdal model, Nucl. Phys. B 416 (1994), 751 [hep-th/9304015].

[58] I.K. Kostov and M. Staudacher, Two-dimensional Chiral matrix models and string theories, Phys. Lett. B 394 (1997), 75 [hep-th/9611011].

[59] L. Lewin, Polylogarithms and associated functions, North-Holland, 1981.

[60] E.T. Whittacker and G.N. Watson, A course in nodern analysis, Cambridge University Press, 1990.

[61] A. Eskin and A. Okounkov, Asymptotics of branched coverings of a torus and volumes of moduli spaces of holomorphic differentials, Invent. Math. 145 (2001), 59 [math.AG/0006171].

[62] M. Kontsevich and A. Zorich, Lyapunov exponents and Hodge theory, Adv. Ser. Math. Phys. 24 (1997), 318 [hep-th/9701164].

[63] M. Kontsevich and A. Zorich, Connected components of the moduli spaces of Abelian differentials with prescribed singularities, Invent. Math. 153 (2003), 631 [math.GT/0201292]. 
[64] A. Eskin, H. Masur and A. Zorich, Moduli spaces of Abelian differentials: the principal boundary, counting problems and the Siegel-Veech constants, Publ. Math. IHES 97 (2003), 61 [math.DS/0202134].

[65] H. Masur, Interval exchange transformations and measured foliations, Ann. Math. 115 (1982), 169.

[66] W. Veech, Gauss measures for transformations on the space of interval exchange maps, Ann. Math. 115 (1982), 201.

[67] C. Vafa, Two dimensional Yang-Mills, black holes and topological strings, hep-th/0406058.

[68] S. Lelli, M. Maggiore and A. Rissone, Perturbative and nonperturbative aspects of the two-dimensional string/Yang-Mills correspondence, Nucl. Phys. B 656 (2003), 37 [hep-th/0211054].

[69] T. Matsuo and S. Matsuura, String theoretical interpretation for finite $N$ Yang-Mills theory in two-dimensions, hep-th/0404204.

[70] R.C. Penner, Perturbative series and the moduli space of Riemann surfaces, J. Diff. Geom. 27 (1988), 35.

[71] P. Hořava, Topological rigid string theory and two-dimensional QCD, Nucl. Phys. B 463 (1996), 238 [hep-th/9507060].

[72] T. Matsuo, S. Matsuura and K. Ohta, Large $N$ limit of $2 D$ Yang-Mills theory and instanton counting, hep-th/0406191.

[73] N.A. Nekrasov, Seiberg-Witten prepotential from instanton counting, Adv. Theor. Math. Phys. 7 (2004), 831 [hep-th/0206161].

[74] N.A. Nekrasov and A. Okounkov, Seiberg-Witten theory and random partitions, hep-th/0306238.

[75] G. Bertoldi, S. Bolognesi, M. Matone, L. Mazzucato and Y. Nakayama, The Liouville geometry of $\mathcal{N}=2$ instantons and the moduli of punctured spheres, JHEP 0405 (2004), 075 [hep-th/0405117].

[76] T. Ishikawa, S.-I. Kuroki and A. Sako, Non-commutative cohomological field theory and GMS soliton, J. Math. Phys. 43 (2002), 872 [hep-th/0107033].

[77] H. García-Compeán, O. Obregón, C. Ramírez and M. Sabido, Noncommutative topological theories of gravity, Phys. Rev. D 68 (2003), 045010 [hep-th/0210203].

[78] H. García-Compeán and P. Paniagua, A non-commutative deformation of topological field theory, hep-th/0402169.

[79] R. Gopakumar, J. Maldacena, S. Minwalla and A. Strominger, S-duality and non-commutative gauge theory, JHEP 6 (2000), 36 [hep-th/0005048]. 
[80] I.R. Klebanov and J. Maldacena, $1+1$ dimensional NCOS and its $U(N)$ gauge theory dual, Int. J. Mod. Phys. A 16 (2001), 922 [hep-th/0006085].

[81] Ph. Flajolet, Singularity analysis and asymptotics of Bernoulli sums, Theor. Comp. Sci. 215 (1999), 371.

[82] I.M. Gradshteyn and I.M. Ryzhik, Table of integrals, series and products, Academic Press, 1980.

[83] R.P. Stanley, Enumerative combinatorics, Wadsworth \& Brooks, 1986.

[84] S. Bloch and A. Okounkov, The character of the infinite Wedge representation, Adv. Math. 149 (2000), 1 [alg-geom/9712009].

[85] B.D. Simons, P.A. Lee and B.L. Altshuler, Exact description of spectral correlators by a quantum one-dimensional model with inverse-square interaction, Phys. Rev. Lett. 70 (1993), 4122.

[86] E.R. Mucciolo, B.S. Shastry, B.D. Simons and B.L. Altshuler, Exact dynamical correlations of the $1 / r^{2}$ model, cond-mat/9309030.

[87] J.A. Minahan and A.P. Polychronakos, Density correlation functions in Calogero-Sutherland models, Phys. Rev. B 50 (1994), 4236 [hep-th/9404192]. 